\title{
Markov Chain Monte Carlo and the Application to Geodetic Time Series Analysis
}

\author{
German Olivares-Pulido, Felix Norman Teferle and Addisu Hunegnaw
}

\begin{abstract}
The time evolution of geophysical phenomena can be characterised by stochastic time series. The stochastic nature of the signal stems from the geophysical phenomena involved and any noise, which may be due to, e.g., un-modelled effects or measurement errors. Until the 1990's, it was usually assumed that white noise could fully characterise this noise. However, this was demonstrated to be not the case and it was proven that this assumption leads to underestimated uncertainties of the geophysical parameters inferred from the geodetic time series. Therefore, in order to fully quantify all the uncertainties as robustly as possible, it is imperative to estimate not only the deterministic but also the stochastic parameters of the time series. In this regard, the Markov Chain Monte Carlo (MCMC) method can provide a sample of the distribution function of all parameters, including those regarding the noise, e.g., spectral index and amplitudes. After presenting the MCMC method and its implementation in our MCMC software we apply it to synthetic and real time series and perform a cross-evaluation using Maximum Likelihood Estimation (MLE) as implemented in the CATS software. Several examples as to how the MCMC method performs as a parameter estimation method for geodetic time series are given in this chapter. These include the applications to GPS position time series, superconducting gravity time series and monthly mean sea level (MSL) records, which all show very different stochastic properties. The impact of the estimated parameter uncertainties on sub-sequentially derived products is briefly demonstrated for the case of plate
\end{abstract}

German Olivares-Pulido

Geoscience Australia, National Positioning Infrastructure Positioning and Community Safety Division, Cnr Jerrabomberra Avenue and Hindmarsh Drive Symonston ACT 2609 GPO Box 378

Canberra ACT 2601 Australia, e-mail: german.olivares@ga.gov.au

Felix Norman Teferle

University of Luxembourg, Geodesy and Geospatial Engineering, 6, rue Richard CoudenhoveKalergi, L-1359 Luxembourg, Grand-Duchy of Luxembourg e-mail: norman.teferle@uni.lu

Addisu Hunegnaw

University of Luxembourg, Geodesy and Geospatial Engineering, 6, rue Richard CoudenhoveKalergi, L-1359 Luxembourg, Grand-Duchy of Luxembourg e-mail: addisu.hunegnaw@uni.lu 
motion models. Finally, the MCMC results for weekly downsampled versions of the benchmark synthetic GNSS time series as provided in Chapter 2 are presented separately in an appendix.

Key words: Time Series Analysis, Stochastic Properties, Markov Chain Monte Carlo, Random-Walk Metropolis-Hasting, Parameter Estimation, Parameter Uncertainties, Geodesy and Geophysics

\section{Introduction}

The dynamics of different geophysical phenomena can be inferred by means of data provided by space-geodetic techniques as, e.g., Doppler Orbitography and Radiopositioning Integrated by Satellite (DORIS) (Cazenave et al., 1992; Lefebvre et al., 1996; Willis et al., 2010), Satellite Laser Ranging (SLR) (Pearlman et al., 2002; Bloßfeld et al., 2018), Very Long Baseline Interferometry (VLBI) (Schlüter et al., 2002; Nothnagel et al., 2017), and Global Navigation Satellite Systems (GNSS) such as the Global Positioning System (GPS) (Beutler et al., 1999; Dow et al., 2009; Teferle et al., 2009; Klos et al., 2018a). These techniques are usually used along with others such as, for example, continuous measurements of near surface movements from strainmeters (Wyatt, 1982, 1989; Langbein et al., 1993), and gravity measurements (Van Camp et al., 2005; Van Camp et al., 2017). They provide data that allow scientists to constrain geophysical models and, in turn, help to better understand phenomena such as, tectonic plate motions (Larson and Agnew, 1991; Fernandes et al., 2004), glacial isostatic adjustments (GIA) (Milne et al., 2001; Bradley et al., 2009), seismic and inter-seismic crustal movements (Prawirodirdjo et al., 1997; Argus et al., 2005), hydrological processes (van Camp et al., 2006; Nahmani et al., 2012) and atmospheric dynamics (Virtanen, 2004; Teke et al., 2011).

In all the above applications it is essential to analyse time series of observations or some kind of derived quantities, such as position estimates from GNSS. While for some applications it is the linear long-term movement derived from position time series that is of interest (Fernandes et al., 2004; Bradley et al., 2009), for others it is the non-linear and periodic displacements that want to be studied (Khan et al., 2008; Nielsen et al., 2013). Nevertheless, since the late 1990s it has become clear that geodetic time series need to be described by both deterministic and stochastic models in order to obtain the best parameter estimates and avoid overly optimistic parameter uncertainties (Langbein and Johnson, 1997; Zhang et al., 1997; Mao et al., 1999; Caporali, 2003; Williams, 2003a; Langbein, 2004; Williams et al., 2004; Williams and Willis, 2006; Langbein, 2008; Teferle et al., 2008; Bos et al., 2010, 2012; Santamaría-Gómez et al., 2012; Klos et al., 2018b). The latter, in particular, may affect studies of long-term changes due to, e.g., geodynamic processes or climate change, where only small changes, e.g., at the millimetre per year level, are expected over many years. Over the last two decades the field of geodetic time series analysis has evolved substantially which is reflected in many publications and the fact that this 
book exists. The GPS Coordinate Time Series Analysis software (CATS) (Williams, 2008), using Maximum Likelihood Estimation (MLE), has been widely used within the community to fit both deterministic and stochastic models to GPS position time series (Teferle et al., 2002, 2008), gravity time series (Van Camp et al., 2005) and mean sea level records (Hughes and Williams, 2010; Burgette et al., 2013). Here we develop a Markov Chain Monte Carlo (MCMC) method with similar applications in mind and investigate its benefits and drawbacks when compared to MLE as implemented in CATS.

\section{Markov Chain Monte Carlo as a Parameter Estimation Method}

\subsection{Fundamentals}

Statistical analysis of geophysical data can be performed in two different ways: from a full knowledge of the parameter space, which is equivalent to having the distribution function, or from a data sample that accounts for the estimation of the distribution function.

A full computation of the parameter space can be performed by mesh-like exploration methods. However, when the number of parameters is high, the computational loading can be overwhelming. Under such circumstances, methods that estimate the distribution function are better than the mesh-like ones. One of such methods is the Markov Chain Monte Carlo (MCMC) method. The theory of Markov chains is well developed and further information can be found in Gilks et al. (1996).

A Markov chain is a series of random variables $X^{(0)}, X^{(1)}, X^{(2)}, \ldots$, in which the influence of the values of $X^{(0)}, X^{(1)}, X^{(2)}, \ldots, X^{(n)}$ on the distribution of $X^{(n+1)}$ is mediated by the value of $X^{(n)}$. More formally,

$$
P\left(X^{(n+1)} \mid X^{(0)}, X^{(1)}, X^{(2)}, \ldots, X^{(n)}\right)=P\left(X^{(n+1)} \mid X^{(n)}\right),
$$

where $P(X)$ denotes the probability of $X$, i.e. the probability that the value for the state variable $x$ is $X$.

A Markov chain can be specified by giving the marginal distribution for $X^{(0)}$ the initial probabilities of the various variables - and the conditional distributions for $X^{(n+1)}$ given the possible values of $X^{(n)}$ - the transition probabilities for one state to follow another state. Henceforth, we will write the initial probability of state $x$ as $p_{0}(x)$, and the transition probability for state $x^{\prime}$ at time $n+1$ to follow state $x$ at time $n$ as $T_{n}\left(x, x^{\prime}\right)$. In our case the transition probabilities do not depend on time (as we shall see later), so we will simply write $T\left(x, x^{\prime}\right)$.

Using the transition probabilities, one can find the probability of state $x$ occurring at time $n+1$, denoted by $p_{n+1}(x)$, from the corresponding probabilities at time $n$, as follows:

$$
p_{n+1}(x)=\sum_{x^{\prime}} p_{n}\left(x^{\prime}\right) T\left(x^{\prime}, x\right)
$$


where the summation goes over all possible states $x^{\prime}$ at time $n$. Given the initial probabilities, $p_{0}$, this determines the behaviour of the chain at all times.

We are interested in constructing Markov chains of which the distribution function, given by $\pi$, is invariant. For this, we will use time reversible homogeneous Markov chains that satisfy the more restrictive condition of detailed balanced - that if a transition occurs from a state picked according the probabilities given by $\pi$, then the probability of that transition being from state $x$ to state $x^{\prime}$ is the same probability of it being from state $x^{\prime}$ to state $x$. In other words, for all $x$ and $x^{\prime}$,

$$
\pi(x) T\left(x, x^{\prime}\right)=\pi\left(x^{\prime}\right) T\left(x^{\prime}, x\right),
$$

which implies that $\pi$ is an invariant distribution, since

$$
\sum_{x^{\prime}} \pi\left(x^{\prime}\right) T\left(x^{\prime}, x\right)=\sum_{x^{\prime}} \pi(x) T\left(x, x^{\prime}\right)=\pi(x) \sum_{x^{\prime}} T\left(x, x^{\prime}\right)=\pi(x),
$$

where we have assumed that $T\left(x, x^{\prime}\right)=1$.

For our purposes, it is not enough to find a Markov chain of which the distribution is invariant. We also require that the Markov chain to be ergodic - that the probabilities at time $\mathrm{n}, p_{n}(x)$, converge to this invariant distribution as $n \rightarrow \infty$, regardless of the choice of the initial probabilities $p_{0}(x)$. Clearly, an ergodic Markov chain can have only one invariant distribution, which is also referred to as its equilibrium distribution.

Though we make use of an ergodic Markov chain there is no way to know how long it takes to reach the equilibrium state. Nevertheless, we can estimate quite well when it is very close (as close as we want, in fact) to such an equilibrium state.

\subsection{The Random-Walk Metropolis-Hasting Algorithm}

One of the simplest MCMC is the so-called Metropolis-Hasting algorithm (Metropolis et al., 1953), where starting at a point $x$ of the Markov chain, a new one $x^{\prime}$ is then accepted with probability $\alpha\left(x, x^{\prime}\right)$ given by

$$
\alpha(x, y)= \begin{cases}\min \left\{\frac{\pi(y)}{\pi(x)} \frac{T(y, x)}{T(x, y)}, 1\right\} & \begin{array}{r}
\pi(x) T(x, y)>0 \\
1,
\end{array}(x) T(x, y)=0,\end{cases}
$$

where $\min (a, b)$ stands for the lowest number of the pair $(a, b)$.

One special case is the Random-Walk Metropolis-Hastings (RWMH) algorithm. In this case, the Markov chain depends on $T\left(x, x^{\prime}\right)=T\left(x-x^{\prime}\right)$ and it behaves as a random-walk process as it only depends on the the difference between two consecutive points of the chain. Thus, Eq. (5) simplifies to

$$
\alpha(x, y)=\min \left\{\frac{\pi(y)}{\pi(x)}, 1\right\} .
$$


Defining the acceptance rate (AR) of any Markov chain as the ratio of the number of accepted points (those from the sample of $\pi(x)$ ) and the length of the chain, and following Roberts and Rosenthal (2001), it can be proved that, as the dimension of the space wherein the RWMH chain lies increases, the optimal step size steers the AR to asymptotically converge to $\sim 0.234$. While some argue that the optimal AR of 0.234 may only be valid for Gaussian densities and not for non-linear models, we find that in all the below applications we generally obtained good results.

The AR is a logistic function of the step size (ss), which is proportional to $\left(x-x^{\prime}\right)$. Indeed, as the step size goes to zero all the proposed values of the chain can be accepted, i.e. AR $\sim 1$, whereas for step sizes too long, the new state $x$ may lie too far away from the maxima of the distribution function, hence it is not accepted, or, in other words, $\mathrm{AR} \sim 0$. Consequently, a linear fit of $\log (\mathrm{AR})$ and $\log (\mathrm{ss})$ can provide the optimal value of the latter in order to get $\mathrm{AR} \sim 0.234$. With that automatic estimate of the step size we can improve efficiency and save a lot of time, as the amount and variability of the time series to be analysed can be huge, and for each of them, the ss can be quite different.

\subsection{The Markov Chain Monte Carlo Algorithm}

The expected values of the estimated parameters are conditioned to the observational data. Note that there is only one data set per station as we can not replicate the observations, but it is possible to simulate as many parameter values as needed. So actually, it is the probability function of recovering the observational data given a parameter set that can be sampled. According to the Bayes theorem the former is related with the probability function of the parameters set of dimension $N, \boldsymbol{\theta} \equiv$ $\left(\theta_{1}, \ldots \theta_{N}\right)$, given an observational data set, $M^{o b s}$, as follows:

$$
\mathcal{P}\left(\boldsymbol{\theta} \mid M^{\text {obs }}\right)=\mathcal{L}\left(M^{\text {obs }} \mid M^{\text {th }}(\boldsymbol{\theta})\right) \mathcal{P}(\boldsymbol{\theta}),
$$

where $M^{\text {th }}(\boldsymbol{\theta})$ is the theoretical magnitude that depends on the value of the parameters set $\boldsymbol{\theta}, \mathcal{L}$ is the Likelihood and it yields the probability of the data given the parameters, and, finally, $\mathcal{P}(\boldsymbol{\theta})$ is the a priori probability function of the parameters.

Once the likelihood is well determined, along with the priors, the posterior distribution, $\mathcal{P}\left(\alpha \mid M^{o b s}\right)$, can be computed. Then the expected values of the parameters of the model can be estimated by means of Monte Carlo integration as follows:

$$
<\theta_{i}>=\int \mathcal{L}(\boldsymbol{\theta}) \theta_{i} d \theta_{j}=\sum_{t=1, T} \frac{\theta_{t, i}}{T}, \forall j \neq i,
$$

where $T$ is the number of points in the chain and $\alpha_{t, i}$ denotes the value of the parameter $\alpha_{i}$ at the $t-t h$ step of the chain. The 100(1-2p)\% confidence interval $\left[c_{p}, c_{1-p}\right]$ for a parameter is estimated by setting $c_{p}$ to the $p^{t h}$ quantile of $\alpha_{t, i}$, $t=1, . ., T$ and $c_{1-p}$ to the $(1-p)^{t h}$ quantile. 
The algorithm that steers the MCMC through the posterior distribution surface is summarised below:

1. Start with a set of parameters $\left\{\alpha_{i}\right\}$, compute the trend (linear and seasonal) and the likelihood $\mathcal{L}_{i}=\mathcal{L}\left(y^{i} \mid y^{o b s}\right)$, where $y^{i}$ is the $i^{\text {th }}$ model, and $y^{o b s}$ the observed data.

2. Take a random step in parameter space to obtain a new set of parameters $\left\{\alpha_{i+1}\right\}$. The probability distribution of the step is taken to be Gaussian in each direction $i$ with the r.m.s given by $\sigma_{i}$. We will refer to $\sigma_{i}$ as the step size. The choice of this step size is important in order to optimise the chain efficiency.

3. Compute the $y_{i+1}$ model for the new set of parameters $\left\{\alpha_{i+1}\right\}$ and their likelihood $\mathcal{L}_{i+1}$.

4. If $\mathcal{L}_{i+1} / \mathcal{L}_{i}>1$, take the step, i.e. save the new set of parameters $\left\{\alpha_{i+1}\right\}$ as part of the chain, then go to step 2 after the substitution $\left\{\alpha_{i}\right\} \rightarrow\left\{\alpha_{i+1}\right\}$.

5. If $\mathcal{L}_{i+1} / \mathcal{L}_{i}<1$, draw a random number $x$ from a uniform distribution from 0 to 1. If $x>\mathcal{L}_{i+1} / \mathcal{L}_{i}$ do not take the step, i.e. save the parameter set $\left\{\alpha_{i}\right\}$ as part of the chain and return to step 2. If $x<\mathcal{L}_{i+1} / \mathcal{L}_{i}$ take the step, i.e. do as in 4 .

6. When the Markov chain achieves the equilibrium state (explained in section 3.1) and the chains have enough points to provide reasonable samples from the posterior distributions, i.e. enough points to reconstruct the $1 \sigma$ and $2 \sigma$ levels of the marginalised likelihood for all the parameters, the chains are stopped.

This algorithm admits values for which $\mathcal{L}_{i+1} / \mathcal{L}_{i}<1$ provided the condition $x<\mathcal{L}_{i+1} / \mathcal{L}_{i}$ holds. Therefore, as it does not guarantee convergence towards the maximum value of the likelihood but rather to a region wherein the maximum lies. It is not an asymptotically consistent maximum likelihood estimator. Consequently, with the fifth condition of the algorithm the MCMC method is not a Maximum Likelihood Estimation (MLE) method.

\section{General Considerations for Markov Chain Monte Carlo}

Let us consider a general power-law spectrum such as (Agnew, 1992)

$$
P(f)=P_{0}\left(\frac{f_{0}}{f}\right)^{-\kappa}
$$

where $P_{0}$ and $f_{0}$ are constant, $f$ is the frequency and $-\kappa$ the spectral index.

We note here that throughout this chapter, the spectral index has different sign with respect to other chapters of this book, i.e. Flicker noise corresponds to $-\kappa=1$.

As Hosking (1981) showed that all times series with $-\kappa>1$ are non-stationary, thus there is not an uniqueness relationship between the covariance matrix and any sample vector of length $T$. In other words, the zeroth and first statistical moments (i.e. the mean and the covariance) are not the only non-zero moments. Moreover, the covariance may evolve in time. 
In Fig. (1) the spectral index $-\kappa$, the power-law amplitude $\sigma_{p l, 0}$, the linear trend $V_{0}$ and the ordinate $y_{0}$ of two time series are the same (all in arbitrary units), hence they have the same covariance matrix, though their evolutions are quite dissimilar. Therefore, an estimation of the velocities by means of the sample recovery of the posterior distribution function would yield different values.

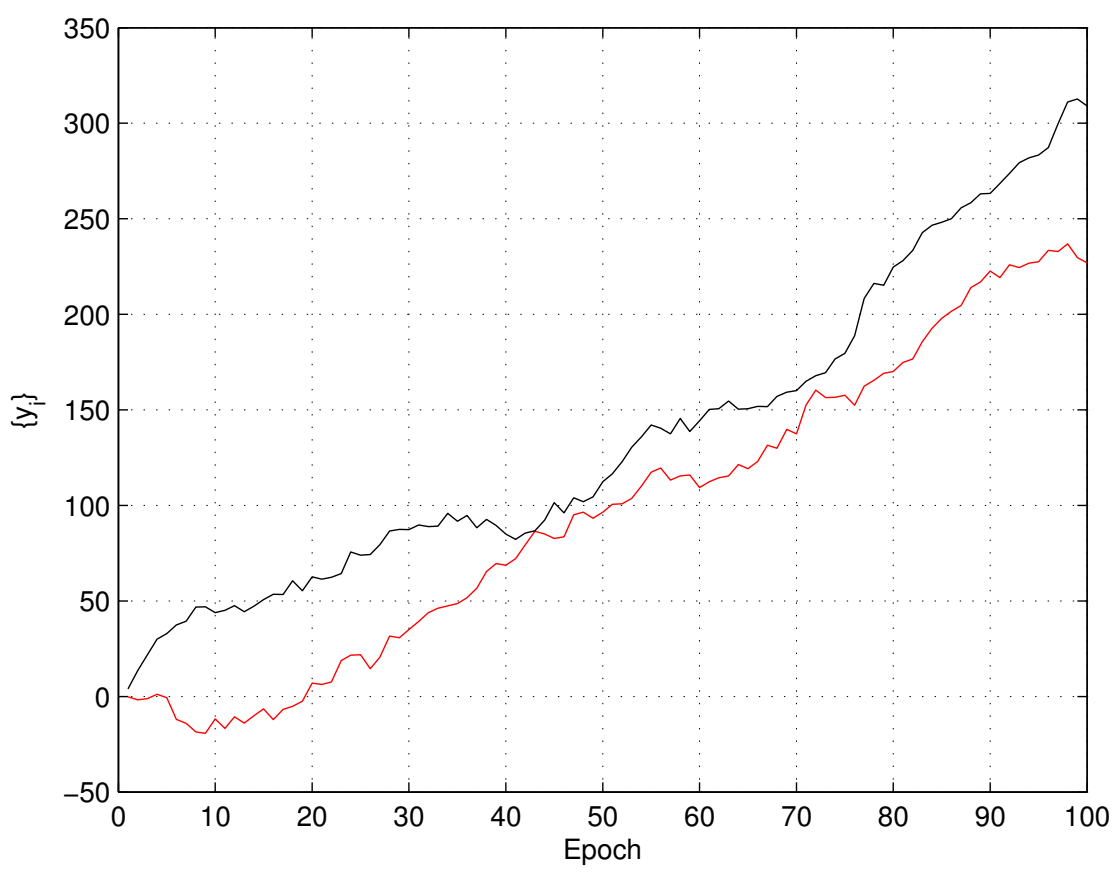

Fig. 1: Two non-stationary time series generated with the same model parameters: $-\kappa_{0}=2.3, \sigma_{p l, 0}=4.5$ and $V_{0}=3.1$.

This is observed in Fig. 2, where the histogram for every parameter is shown. In Fig. (2d), there are several local maxima for the velocity. As a consequence, the estimation of the linear trend might not be uniquely determined for non-stationary processes. Nevertheless, by computing the first difference any non-stationary time series (provided $-\kappa<2$ ) can be transformed into a stationary one, thus the slope would come exclusively from non-stochastic processes.

A similar bias effect on the slope estimation due to seasonal processes was already noticed by Blewitt and Lavallée (2002). The difference is that the seasonal bias effect 

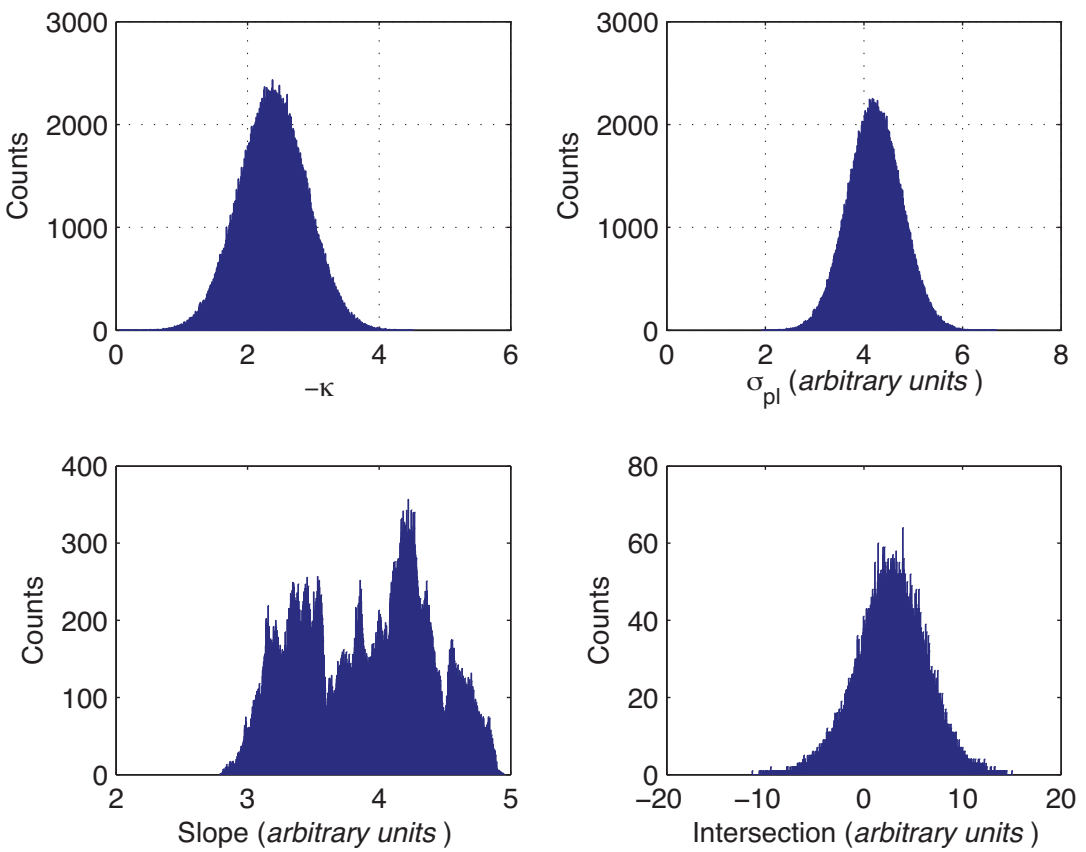

Fig. 2: Histograms of the estimated parameters with $-\kappa_{0}=2.3, \sigma_{p l, 0}=4.5$ and $V_{0}=3.1$ as true values.

decreases as the time series length increases, whereas it does not for non-stationary stochastic processes unless they have been differenced beforehand.

\subsection{The Equilibrium State}

The equilibrium or stationary state of the MCMC method implies that the estimates of the parameters can not be significantly improved (from the statistical standpoint by obtaining more points of the distribution function). There are some common characteristics of the stationary state to take into account:

- For a RWMH algorithm the acceptance rate (AR) or sample density should be 0.234 (Roberts and Rosenthal, 2001).

- The spectrum of the Markov chain at low frequency should be flat, meaning that there are no correlations or these are damped enough for the points to be considered independent of each other, thus minimising any bias on the estimates. 


\subsection{The Acceptance Rate}

The $A R$, which is defined as the ratio between the points from the sample and all the points tried by the Markov chain, depends mainly on the step size. If the step size is too long, it could yield a low acceptance rate; if it is too short, the sample data would not be well mixed, i.e. they might have a big correlation, though the acceptance rate would be high. So, good convergence and good mixing is achieved with a trade off on the step size.

The $A R$ is a logistic function of the inverse of the step size $1 / s s$ :

$$
A R(s)=\frac{1}{1+\gamma e^{-1 / s s}},
$$

with $\gamma$ being a non-dimensional parameter that controls the transition from low to high values of the argument of the logistic function. Indeed, Fig. 3 shows that for small step sizes, i.e. big $1 / s s$ values, most of the the proposed values of the chain are accepted, i.e. $A R \sim 1$, whereas for step sizes too long (small 1/ss values) $A R \sim 0$. According to that, a linear fit of $\log (A R)$ and $\log (s s)$ can estimate the optimal value for the latter in order to get $A R \sim 0.234$ (horizontal vertical line in 3 ), which is a necessary condition for an optimal performance of the MCMC method so as to obtain an unbiased sample of the parameters distribution.

\subsection{The Spectrum of the Markov Chain}

At small scale, the Markov chain is mainly a random-walk process, therefore the points therein are correlated and any estimate for the parameters at that scale will be biased. In order to get a non-biased estimate, the Markov chain has to reach the stationary state and a good mixing. By the ergodic theorem (Gilks et al., 1996), the Markov chain at long-scale provides a homogeneous sample of the distribution function, i.e. when its length is long enough it yields a white-noise-like spectrum. Following Dunkley et al. (2005) this spectrum can be written as

$$
P(k)=P_{0} \frac{\left(k^{*} / k\right)^{\beta}}{\left(k^{*} / k\right)^{\beta}+1},
$$

where $\beta>0$ is the spectral index of the spectrum of the Markov chain (it has nothing to do with the spectral index $-\kappa$ of the time series itself $), k=j(2 \pi / M)$ is the scale (with $j \in \mathbf{N}$ ), $M$ the length of the chain, $k^{*}$ the cross-over scale, i.e. the inverse of the length for which two points of the Markov chain that distance apart (at least) are uncorrelated, and $P_{0}=P(k \rightarrow 0)$. 


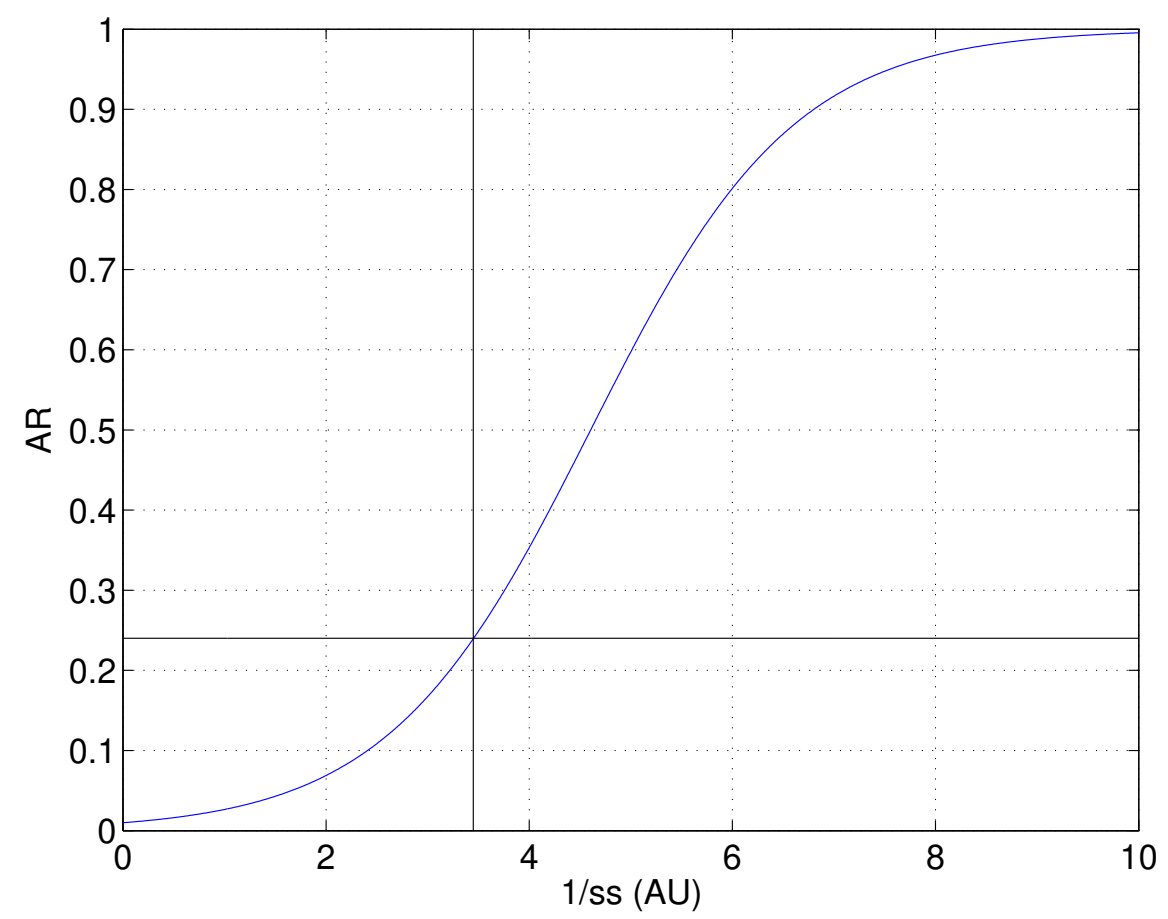

Fig. 3: Logistic function for $A R$ vs. the inverse of the step size 1/ss (in AU). Black solid lines correspond to $A R=0.234$ (horizontal) and optimal step size $s s=0.30 A U$ (vertical).

Fig. 4 shows the theoretical spectrum above for a long Markov chain obtained from a Random-Walk Metropolis-Hasting algorithm. The values of the parameters are $P_{0}=100, \beta=2$ and $k^{*}=1$ (all in AU). At low frequency $\left(k<k^{*}\right)$ there is a plateau, meaning that points in the Markov chain at distances longer than $1 / k^{*}$ are uncorrelated. On the other hand, at high frequency $\left(k>k^{*}\right)$ the points are correlated, giving rise to biased parameter estimates. Therefore, the spectral analysis of the Markov chain provides a tool to estimate the length for which the bias on the estimated parameters is minimised.

According to Dunkley et al. (2005), the Markov chain has a good mixing when $k_{\text {min }} \equiv 1 / M$ is in the white noise regime, i.e. $k_{\text {min }}<k^{*}$, as it guarantees that the chain is long enough to minimize correlations between Markov chain points, hence obtaining unbiased estimates.

Finally, as it takes some time for the chain to achieve the stationary state which guarantees the ergodic theorem to hold (Gilks et al., 1996), 33\% of the first points are usually burned. 


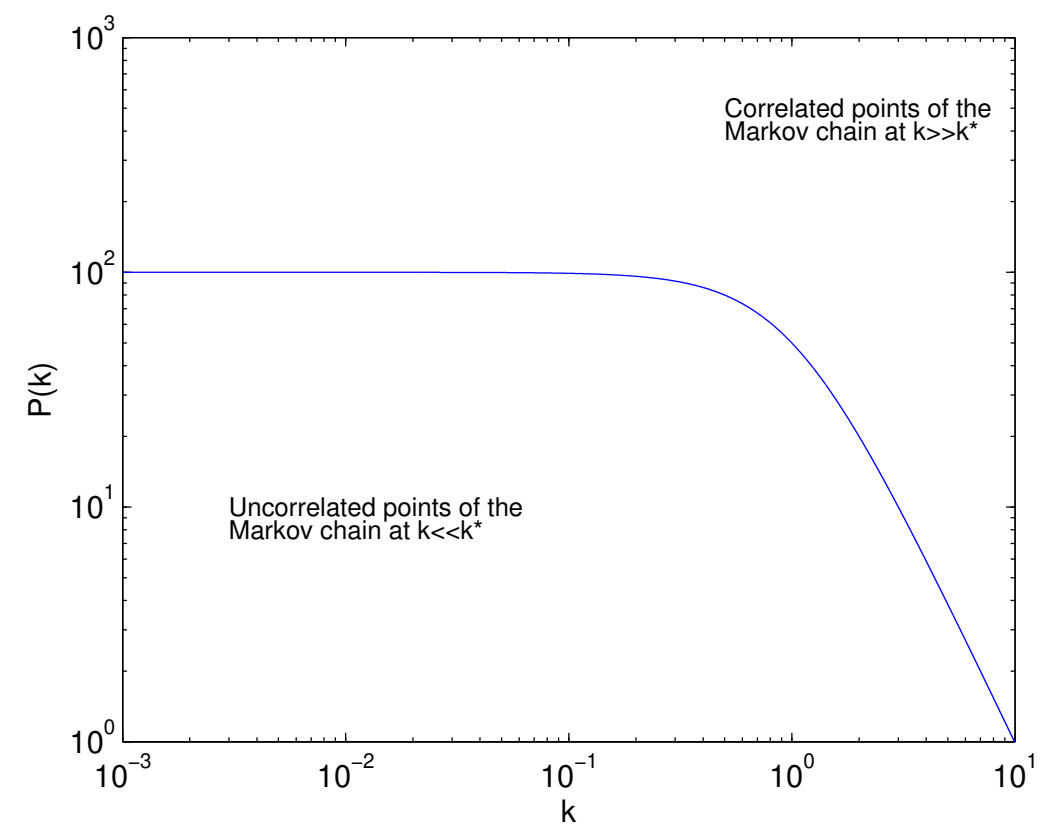

Fig. 4: Power spectrum for long Markov chains of Random-Walk Metropolis-Hasting algorithms.

\section{Applications}

In this section, time series from different geodetic data sets were analysed using the MCMC method described previously and implemented in our in-house MCMC software. The data sets comprise a set of synthetic time series as well as measurements and solutions from three different geodetic techniques: GPS, superconducting gravity and tide gauge records.

The synthetic time series were generated in order to assess the MCMC method through investigating the ability of the algorithm to recover the input values when generating the time series. Aspects of this analysis were published in Olivares and Teferle (2013).

The analysis of the first real data set consists in applying the MCMC method to GPS position time series from the Jet Propulsion Laboratory (JPL). Moreover, plate motion models were computed using the velocities and uncertainties obtained from the MCMC and MLE methods, the latter as implemented in CATS (Williams, 2008), in order to assess their differences and the impact on the plate-motion model parameter estimates.

The second real data set comprises gravity measurements from the superconducting gravimeter at Membach, Belgium, which are analysed in order to estimate the 
noise and the trend (Van Camp et al., 2005, 2016). These gravity time series show largely different characteristics from the position estimates derived from continuous GPS measurements.

Finally, the third real data set comprises tide gauge records from the Permanent Service of Mean Sea Level (PSMSL) as archived in its Revised Local Reference (LRL) database (Holgate et al., 2013). Again the characteristics of this data set are significantly different from the other two sets. Furthermore, the PSMSL only provides trend estimates based on a white noise only stochastic model.

\subsection{Position Time Series}

In this section we employ daily position time series that were 1) synthetically generated and 2) obtained from the Jet Propulsion Laboratory (JPL) GPS time series website ${ }^{1}$.

\subsubsection{Synthetic Data}

Firstly, 100 synthetic time series with different real parameters are analysed with our MCMC software and CATS (using CATS v3.1.2). Thus we can investigate and describe their common features and differences. We used CATS as the benchmark for the performance of the MCMC method.

A combination of linear and periodic terms, and time-correlated noise with length $N=500$ is considered in order to assess the performance of the new MCMC method:

$$
y(t)=y_{0}+v\left(t-t_{0}\right)+\sum_{k=1}^{k=H}\left(A_{k}^{c} \cos \left(2 \pi f_{k} t\right)+A_{k}^{s} \sin \left(2 \pi f_{k} t\right)\right)+r(t),
$$

where the parameters to be estimated are the ordinate $y_{0}$, the velocity $v$, the periodic amplitudes $A_{k}^{c}$ and $A_{k}^{s}$ of the $k^{t h}$ harmonic, and the stochastic noise $r(t)$ components: the spectral index $-\kappa$, the power amplitude of the power-law process $\sigma_{p l}$ and that of the white noise $\sigma_{w n}$, all of them in artificial units (AU). The frequencies $f_{k}$ of the harmonics, the number of harmonics $H$ and the initial epoch $t_{0}$ are input values. Table 1 shows the true values of the parameters, which are typical of real GPS time series (Zhang et al., 1997; Mao et al., 1999; Williams et al., 2004; Hack1 et al., 2011).

Then, another set of 100 synthetic time series is analysed with MCMC and CATS in order to assess the performance of both, thus highlighting their differences and similarities. For this case, semi-annual terms were also included in Eq. 12. The first time series was generated with the initial true values listed in the second column of

\footnotetext{
${ }^{1}$ http://sideshow.jpl.nasa.gov/post/series.html
} 
Table 1: True values (in AU) of the parameters of the synthetic time series.

\begin{tabular}{lc}
\hline Parameter & True Value \\
\hline$-\kappa$ & 1.10 \\
$\sigma_{p l}$ & 1.00 \\
$\sigma_{w n}$ & 0.20 \\
$v$ & 20.00 \\
$y_{0}$ & 0.00 \\
$A^{c}$ & 10.00 \\
$A^{s}$ & 5.00 \\
\hline
\end{tabular}

Table 2: Initial true values and Gaussian generators of the parameters of the 100 synthetic time series data set. All parameters are in AU.

\begin{tabular}{lcl}
\hline Parameter & Initial true value & Gaussian generator \\
\hline$-_{i}$ & 1.10 & $\mathcal{N}\left(-\kappa_{i-1}, 0.1\right)$ \\
$\sigma_{p l, i}$ & 2.30 & $\mathcal{N}\left(\sigma_{p l, i-1}, 0.01\right)$ \\
$\sigma_{w n, i}$ & 2.00 & $\mathcal{N}\left(\sigma_{w n, i-1}, 0.01\right)$ \\
$v_{i}$ & 20.00 & $\mathcal{N}\left(v_{i-1}, 10\right)$ \\
$y_{0, i}$ & 0.00 & $\mathcal{N}\left(y_{0, i-1}, 0.02\right)$ \\
$A_{1 y r, i}^{c}$ & 1.00 & $\mathcal{N}\left(A_{1 y r, i-1}^{c}, 0.1\right)$ \\
$A_{1 y r}^{s}$ & 5.00 & $\mathcal{N}\left(A_{1 y r, i-1}^{s}, 0.1\right)$ \\
$A_{0.5 y r}^{c}$ & 1.00 & $\mathcal{N}\left(A_{0.5 y r, i-1}^{c}, 0.1\right)$ \\
$A_{0.5 y r}^{s}$ & 2.00 & $\mathcal{N}\left(A_{0.5 y r, i-1}^{s}, 0.1\right)$ \\
\hline
\end{tabular}

Table 2. Then, for the other 99 time series, their true values were generated with a random-walk process starting at the initial true values with Gaussian generators listed in the third column of Table 2.

Fig. 5 shows a synthetic time series representative of the data set. For all of them the period spans around 10 years. The periodic amplitudes have been enhanced (compared, for example, with those in Fig. 11) in order to assess the robustness of both methods.

\section{Parameter Estimates.}

The analysis carried out on the synthetic time series shows that the model parameter estimates from both methods agree very well, with some differences in their stochastic parameters. Ideally, as both implementations assume a Gaussian likelihood, they should lead to equivalent results. Nevertheless, in general, the MCMC software estimates larger $-\kappa$ and $\sigma_{w n}$, and slightly smaller $\sigma_{p l}$ than the MLE implementation in CATS. As for the model parameters, the MCMC method yields larger values for all of them. 


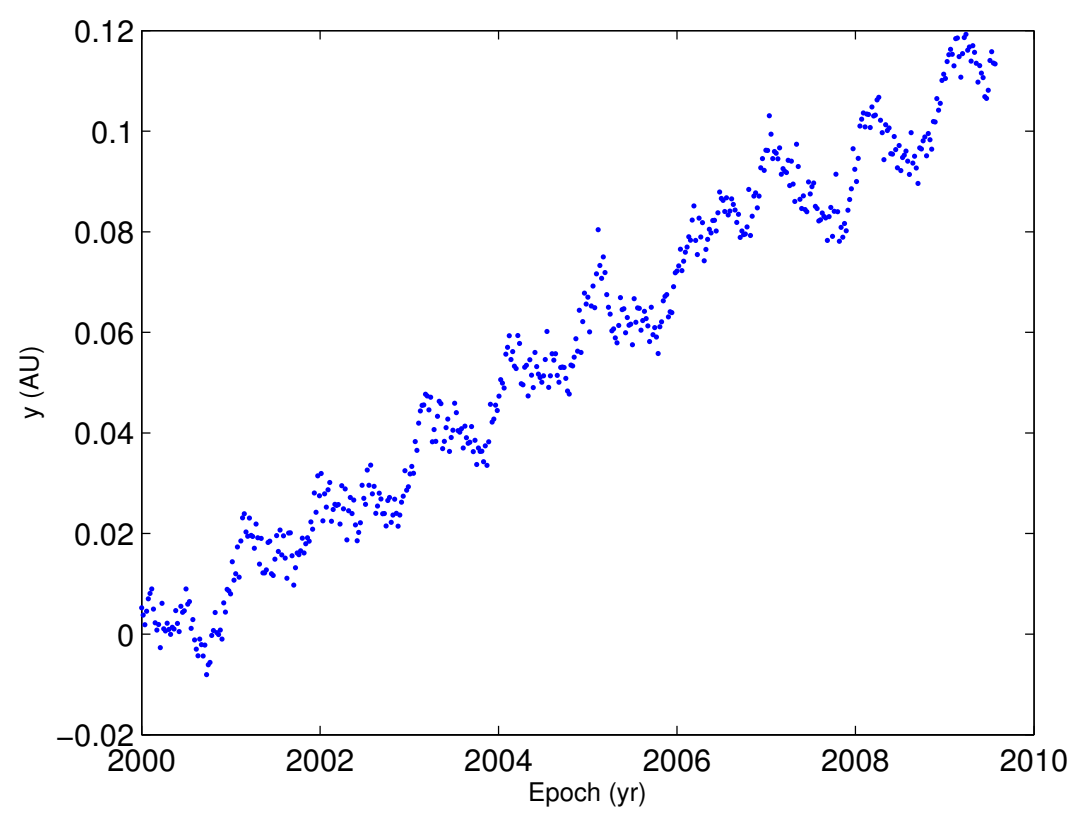

Fig. 5: Synthetic data generated with parameters from Table 2.

The correlation plots in Fig. 6 show the estimates for $-\kappa$ (a), $\sigma_{p l}$ (b) and $\sigma_{w n}$ (c). The $-\kappa$ estimates from MCMC are larger than for CATS, whereas Fig. $6 \mathrm{~b}$ shows larger $\sigma_{p l}$ estimates from CATS. Fig. 6a indicates a systematic bias between the $-\kappa$ estimates of both implementations. These differences are further investigated later in the analysis with real GPS position time series.

A Linear Least Squares (LLS) fit estimates the correlation (the slope of the fit) and the bias (the ordinate of the fit). Thus the following linear relationship between estimates and uncertainties of both methods is assumed

$$
\operatorname{par}_{M C M C}=a \times p a r_{C A T S}+b,
$$

where $\operatorname{par}_{M C M C}\left(\operatorname{par}_{C A T S}\right)$ is either an estimated parameter or its uncertainty from the MCMC (CATS) method, $a$ is the slope, and $b$ the bias. This formula allows transformation of all the analysis carried out with CATS into MCMC values.

Table 3 summarises the results for all parameters but $\sigma_{w n}$. For $-\kappa$, the values of the slope $(a=0.78)$ and the bias $(b=0.37)$ account for the disagreement between both methods at low values of the spectral index, whereas the estimates meet at high values. 
Another analysis carried out on a data set of 100 synthetic time series for which the true value of the spectral index ranges from 0 to 5 supports this value of the slope. Fig. 7 shows the parameter estimates for $-\kappa$ from MCMC vs. CATS of this last synthetic data set. The figure suggests that the estimates agree much better at high values. In this case, $a=0.91$ and $b=0.28$. The better correlation shown at high values in Fig. 7 suggests that, like the MCMC method, CATS is also a good estimator of the spectral index for non-stationary time series (Olivares and Teferle, 2013).

The red-circled points in Fig. 7 are estimates for which CATS sets $\sigma_{w n}=0$. The fact that they cluster at low value suggests that these discrepancies of $-\kappa$ stem from the way CATS deals with low spectral index values. When these are too small, CATS considers there is only power-law noise, thus setting the white noise amplitude equal to zero $^{2}$ (red-circled points along the vertical axis in Fig. 6c). Consequently, the spectral index is whitened in order to account for the amplitude of the white noise process, hence the larger positive bias (marked as red-circled points) in Fig. 6a. Moreover, the power-law amplitude from CATS is shifted up to include the power from the white noise process, thus introducing larger (more negative) bias between both estimates at high values as it is observed in Fig 6b (red-circled points).

As for the model parameters, the correlation plots in Fig. 8 show that the estimates from both methods agree very well. Apart from a few outliers (any point beyond $3 \sigma$ confidence level), which come either from a Markov chain that did not achieved the stationary state, or from numerical issues in CATS, the data cluster along the diagonal. Their values for the LLS fit parameters in Table 3 also show good agreement with $a \sim 1$ for all and, in general, small biases, except for $A_{1 y r}^{s}$.

In order to numerically assess their agreements, the RMS with respect to the true value was computed for both methods. Table 4 summarises the RMS in AU for the estimates from both methods as implemented in MCMC and CATS. It shows that the RMS for the $-\kappa$ estimates are in good agreement: $R M S=0.20$ and $R M S=0.22$ for the estimate from MCMC and CATS, respectively. Nevertheless, the values from the $\sigma_{p l}$ estimates differ further: $R M S=0.44$ and $R M S=0.51$, i.e. an improvement of $14 \%$ by the MCMC method. And, due to the way CATS sets $\sigma_{w n}=0$ for low values of $\sigma_{p l}$, the RMS from both methods are even more different: $R M S=0.47$ for the MCMC, and RMS = 1.01 for the CATS method, i.e. 53\% smaller for the MCMC than for the CATS method.

On the other hand, the values of the RMS of the estimates of the model parameters shown in Table 4 do not differ that much with $R M S=0.41,0.30,0.30$ for the estimates of $v, A_{0.5 y r}^{c}$ and $A_{0.5 y r}^{s}$, respectively, from the MCMC method; and $R M S=$ $0.40,0.30,0.32$ for their CATS counterparts. The other three model estimates from CATS, though, show better fit, with $R M S=1.61,0.44,0.39$, for $y_{0}, A_{1 y r}^{c}, A_{1 y r}^{s}$,

${ }^{2}$ S.D.P. Williams, personal communication, 2012. 


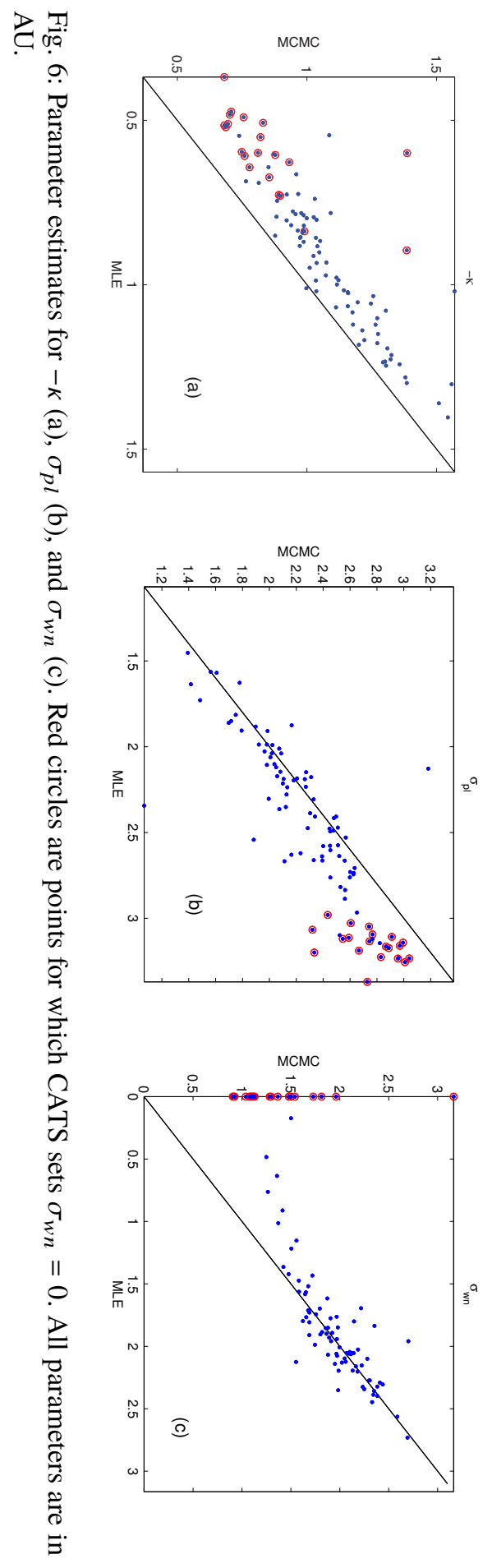




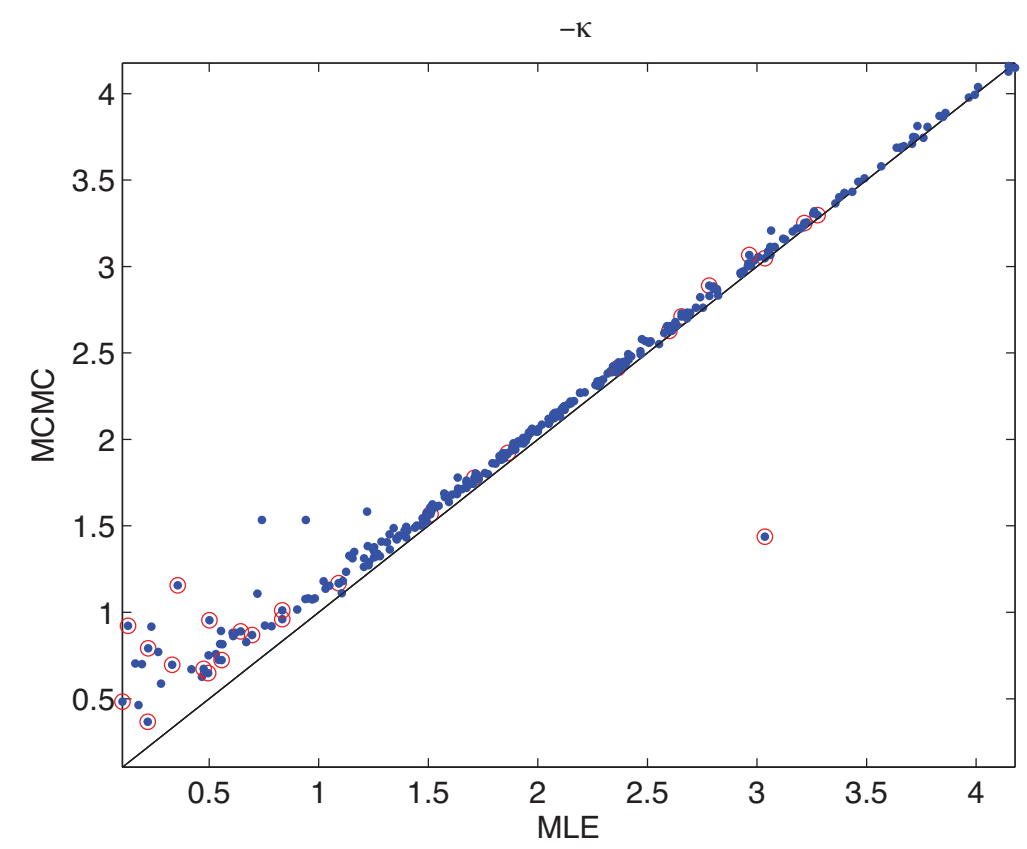

Fig. 7: Parameter estimates for $-\kappa$. Red circles are time series for which CATS sets $\sigma_{w n}=0$.

Table 3: Table of LLS fit parameters, as defined in Eq. 13 (synthetic data).

\begin{tabular}{lll}
\hline Parameter & \multicolumn{2}{c}{$b$} \\
\cline { 2 - 3 } & $a$ & 0.37 \\
\hline$-\kappa$ & 0.78 & 0.46 \\
$\sigma_{p l}$ & 0.75 & -0.03 \\
$\nu$ & 1.00 & 0.14 \\
$y_{0}$ & 0.96 & 0.00 \\
$A_{1 y r}^{c}$ & 1.01 & 0.32 \\
$A_{1 y r}^{s}$ & 0.92 & 0.02 \\
$A_{0.5 y r}^{c}$ & 0.97 & 0.12 \\
$A_{0.5 y r}^{s}$ & 0.93 & \\
\hline
\end{tabular}

respectively; whereas the estimates from the MCMC are larger, namely $R M S=$ $1.76,0.52,0.59$.

To summarise, according to the results for the RMS, both methods perform alike, except for the estimate of the amplitude of the white noise, which is clearly underestimated by the CATS method. 


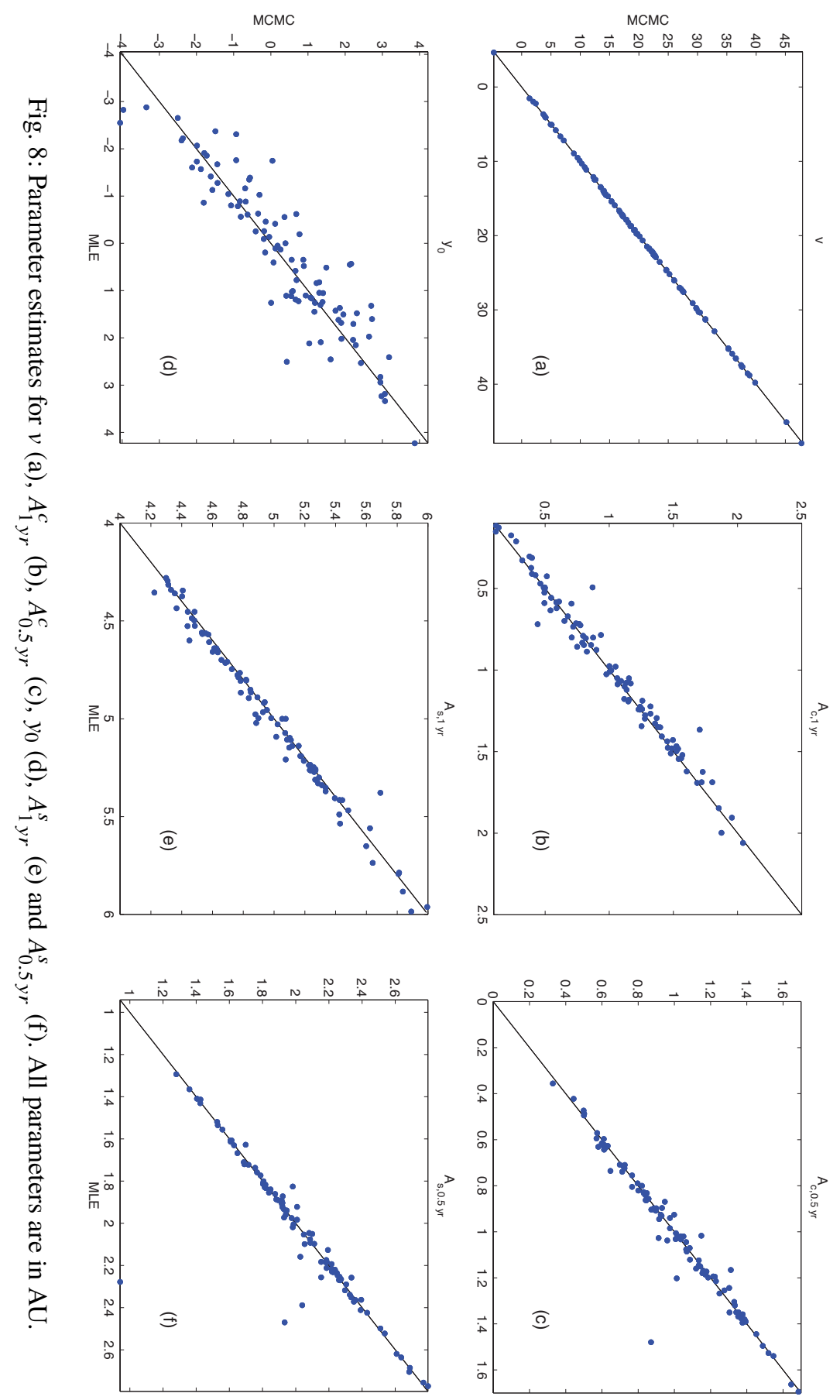


Table 4: RMS values (in AU) for parameter estimates from the MCMC and CATS methods.

\begin{tabular}{lccccccccc}
\hline Method & $-\kappa$ & $\sigma_{p l}$ & $\sigma_{w n}$ & $v$ & $y_{0}$ & $A_{1 y r}^{c}$ & $A_{1 y r}^{s}$ & $A_{0.5 y r}^{c}$ & $A_{0.5 y r}^{s}$ \\
\hline MCMC & 0.20 & 0.44 & 0.47 & 0.41 & 1.76 & 0.52 & 0.59 & 0.30 & 0.30 \\
CATS & 0.22 & 0.51 & 1.01 & 0.40 & 1.61 & 0.44 & 0.39 & 0.30 & 0.32 \\
\hline
\end{tabular}

Table 5: Table of LLS fit parameters, as defined in Eq. 13, for uncertainties (synthetic data). All parameters are in AU.

\begin{tabular}{lll}
\hline Parameter & \multicolumn{2}{l}{} \\
\cline { 2 - 3 } & $a$ & $b$ \\
\hline$\sigma_{v}$ & 1.22 & 0.11 \\
$\sigma_{y_{0}}$ & 0.16 & 1.41 \\
$\sigma_{A_{1 y r}^{c}}$ & 0.62 & 0.40 \\
$\sigma_{A_{1 y r}^{s}}$ & 1.01 & 0.03 \\
$\sigma_{A_{0.5 y r}^{c}}$ & 0.95 & 0.06 \\
$\sigma_{A_{0.5 y r}^{s}}$ & 0.96 & 0.05 \\
\hline
\end{tabular}

\section{Uncertainties.}

Fig. 9 shows the correlation of the uncertainties for the model parameters, i.e. $\sigma_{v}$, $\sigma_{y_{0}}, \sigma_{A_{1 y r}^{c}}, \sigma_{A_{1 y r}^{s}}, \sigma_{A_{0.5 y r}^{c}}$ and $\sigma_{A_{0.5 y r}^{s}}$, from both methods. In general, the velocity (Fig. 9a) and periodic terms (Figs. 9b, 9c, 9e and 9f) uncertainties align along and above the diagonal, meaning that they clearly correlate with larger values from the MCMC method. The exception is $\sigma_{y_{0}}$, in Fig. 9d, for which the uncertainties from the CATS method are larger than from the MCMC method. Besides showing a low correlation, Fig. $9 \mathrm{~d}$ also indicates that for large $\sigma_{y_{0}}$ values, the difference between these from the CATS and MCMC methods increases.

Table 5 summarises the values of the LLS fit that transforms the values from the CATS method. The uncertainties $\sigma_{v}, \sigma_{A_{1 y r}^{s}}, \sigma_{A_{0.5 y r}^{c}}$ and $\sigma_{A_{0.5 y r}^{s}}$ are well correlated along the diagonal with $a=1.22,1.01,0.95$, and 0.96 , respectively, with small biases, namely $b=0.11,0.03,0.06$, and 0.05 . Though $a=0.16$ for $\sigma_{y_{0}}$, its bias is larger, $b=1.41$. The outliers at the head and tail of Fig. $9 \mathrm{~b}$ lead to a smaller slope, $a=0.62$, and higher bias, $b=0.40$, on $\sigma_{A_{1 y r}^{c}}$.

As the parameter space is wider for the MCMC method, it explores the surroundings of the maximum of the likelihood for all parameters, including $-\kappa$, hence the larger uncertainties for all parameters except for $y_{0}$. The stochastic amplitudes $-\kappa$, $\sigma_{p l}$ and $\sigma_{w n}$ estimated from MCMC reduce the RMS (see Table 4), therefore the estimated uncertainties, though larger, provide a more comprehensive estimate of the noise.

Other statistic variables that provide a comprehensive analysis of both methods are the median of the ratio of the uncertainties from both methods of the estimate of $p$, i.e. 


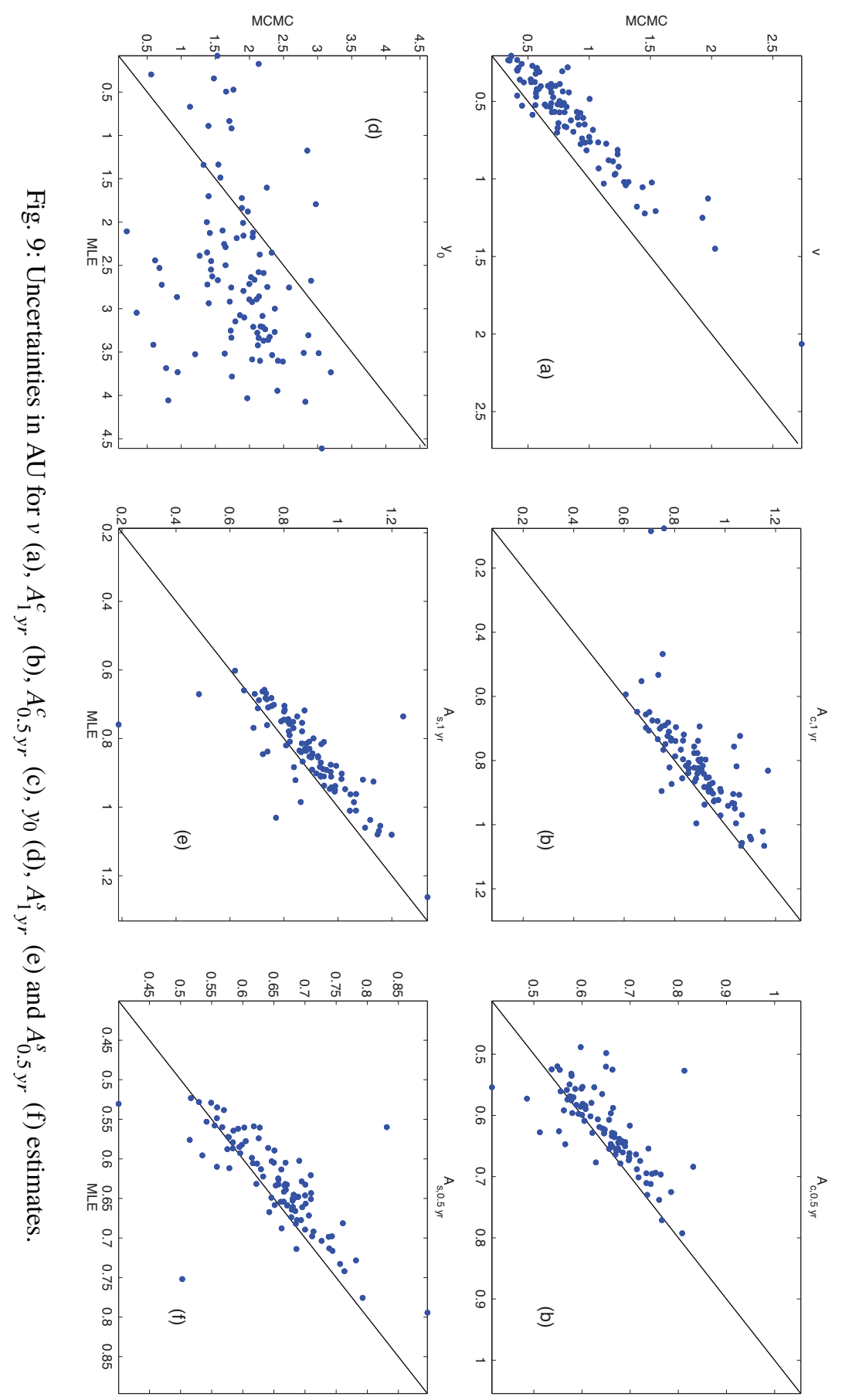


Table 6: Values in $\mathrm{AU}$ of the median of the ratio of the uncertainties $R_{p}$, and the median of the differences $\Delta_{p}$ (synthetic data).

\begin{tabular}{llc}
\hline Uncertainty & $R_{p}$ & $\Delta_{p}$ \\
\hline$\sigma_{v}$ & 1.40 & 0.23 \\
$\sigma_{y_{0}}$ & 0.69 & -0.88 \\
$\sigma_{A_{1 y r}^{c}}$ & 1.08 & 0.06 \\
$\sigma_{A_{1 y r}^{s}}$ & 1.07 & 0.06 \\
$\sigma_{A_{0.5 y r}^{c}}$ & 1.04 & 0.02 \\
$\sigma_{A_{0.5 y r}^{s}}^{s}$ & 1.03 & 0.02 \\
\hline
\end{tabular}

$$
R_{p} \equiv \operatorname{median}\left(\frac{\sigma_{p}^{M C M C}}{\sigma_{p}^{C A T S}}\right),
$$

and the median of the differences, namely

$$
\Delta_{p} \equiv \operatorname{median}\left(p_{M C M C}-p_{C A T S}\right) .
$$

These are listed in Table 6 for the synthetic data. Concerning the ratios (second column in Table 6), the largest absolute median is that for $\sigma_{v}$, namely $R_{v}=1.40$, whereas the smallest is for $y_{0}: R_{y_{0}}=0.69$. On the other hand, the uncertainties of the periodic terms from both methods are quite similar ranging from 1.03 to 1.08. As for the differences (third column in Table 6), the largest one is for the ordinate uncertainty, namely -0.88 . This also makes the largest difference between the bias (third column in Table 5) and $\Delta_{y_{0}}$. For the other parameters their $\Delta_{p}$ values are of the same order of magnitude as their ratio counterparts.

Although $R_{p}\left(\Delta_{p}\right)$ and $a(b)$ (see Table 5) for the velocity and periodic terms estimates are similar, the latter is less robust with outliers, as the large difference between $R_{y_{0}}=0.69\left(\Delta_{y_{0}}=-0.88\right)$ and $a=0.16(b=1.41)$ indicates. Therefore, in order to provide a more robust assessment it is advised to use $R_{p}$ and $\Delta_{p}$ rather than $a$ and $b$ for rescaling from CATS onto MCMC values, as the former provide more robust statistical information.

\subsubsection{Real Data}

At the time of this study JPL provided 2381 daily position time series processed using the Precise Point Position (PPP) strategy in the GIPSY-OASIS II software ${ }^{3}$ (Zumberge et al., 1997). Out of them, 90 GPS stations (shown in Fig. 10) from the International GNSS Service (IGS) have been selected in order to perform the analysis. Fig. 11 shows the North, East and Up components of station ALIC as a representative GPS position time series from this data set. Also shown are the root

${ }^{3}$ The software is available in http://sideshow.jpl.nasa.gov/post/series.html 


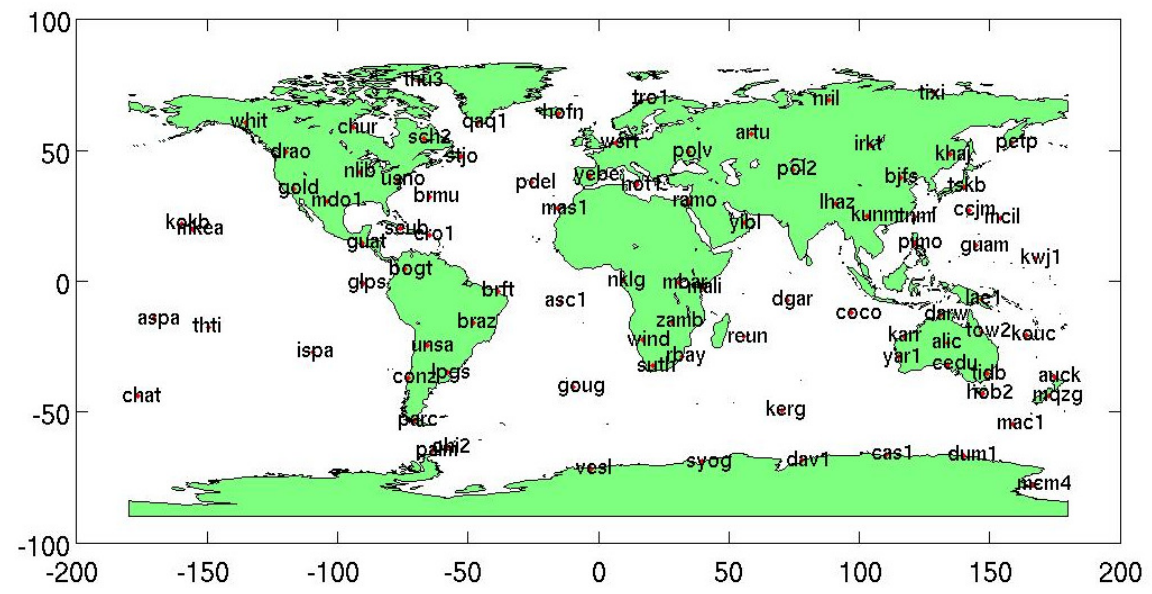

Fig. 10: Map of the GPS stations of the IGS core network.

mean square (RMS) values, which are $1.26 \mathrm{~mm}$ and $1.25 \mathrm{~mm}$ for the North and East components, respectively; whereas for the UP component it is $3.66 \mathrm{~mm}$. These are typical values of the RMS of GPS time series and in this case, but also in general, the RMS for the Up component is $~ 3$ times larger than the RMS for the North and East components.

Alongside the trended (and detrended) time series, JPL also provides the epochs of the discontinuities within the time series. In total JPL reported 4078 offsets for this data set, meaning 1.7 offsets per station on average. As such offsets introduce additional coloured noise (Williams, 2003b), the time series were corrected before the analysis of the stochastic properties. Furthermore, discontinuities in the position time series may have significant affects on the parameter estimates (Williams, 2003b; Gazeaux et al., 2013).

Fig. 12(a) shows the spans of the weekly time series. Note than the spans of JPL time series range from over 6 years to about 19 years. In Fig. 12(b) the histograms of the gaps in the time series show short periods of gaps as more than $87 \%$ of them have less than $25 \%$ of epoch discontinuities, i.e. gaps. Gaps introduce zeroes in the inverse of the covariance matrix thereby making it sparser and shifting the spectral index estimate. The problem with a sparse matrix is that it may have a high conditioning number, thus leading to a biased likelihood when its inverse (i.e. the covariance matrix) is computed.

These 90 daily position time series from the IGS core network were converted into weekly position time series in order to boost the computational speed. Though the 

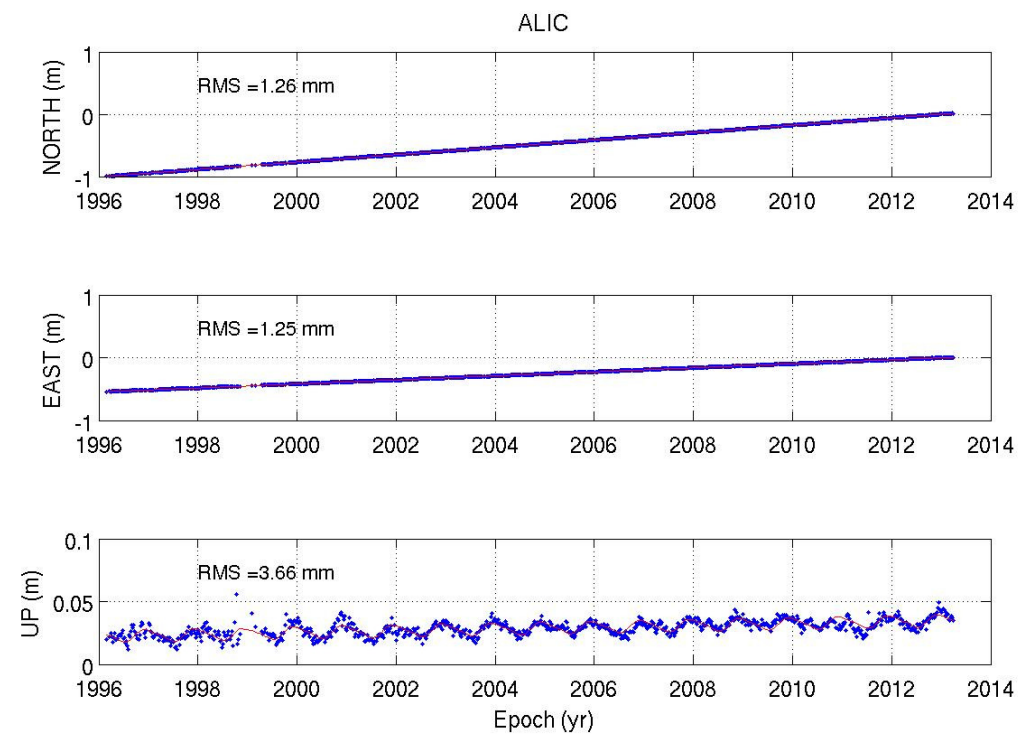

Fig. 11: GPS time series for ALIC. From top to bottom: North, East and Up components.

conversion from daily to weekly time series might modify the stochastic parameters (Kirchner, 2005), it does not affect the comparison between the MCMC and CATS methods, as long as the time series are the same for both.

Parameter Estimates. The results for the analysis of the JPL data set are similar to those for the synthetic data set. In general, $-\kappa$ for the MCMC method is larger than for CATS, whereas the estimated model parameters for both methods agree very well.

On the other hand, $\sigma_{p l}$ for the CATS method is larger than for MCMC, in good agreement with the results for the synthetic time series.

Regarding $\sigma_{w n}$, most of the values for CATS $(82 \%, 77 \%, 70 \%$, for the North, East and Up components, respectively) are set to zero.

According to Figs. 13a, 13b and 13c, the estimates of the spectral index $-\kappa$ for all three components are above the diagonal, i.e. the MCMC method yields larger estimates for this parameter than CATS. On the other hand, Figs. 14a, 14b and 14c show that $\sigma_{p l}$ for all three components are larger for CATS than for MCMC, also in good agreement with the results obtained for the synthetic time series shown in Fig. (6). Nevertheless, as the noise depends geometrically on $-\kappa$ (only linearly on $\sigma_{p l}$ ), and it is larger for MCMC, the uncertainties of the estimates from the MCMC method are expected to be larger. 

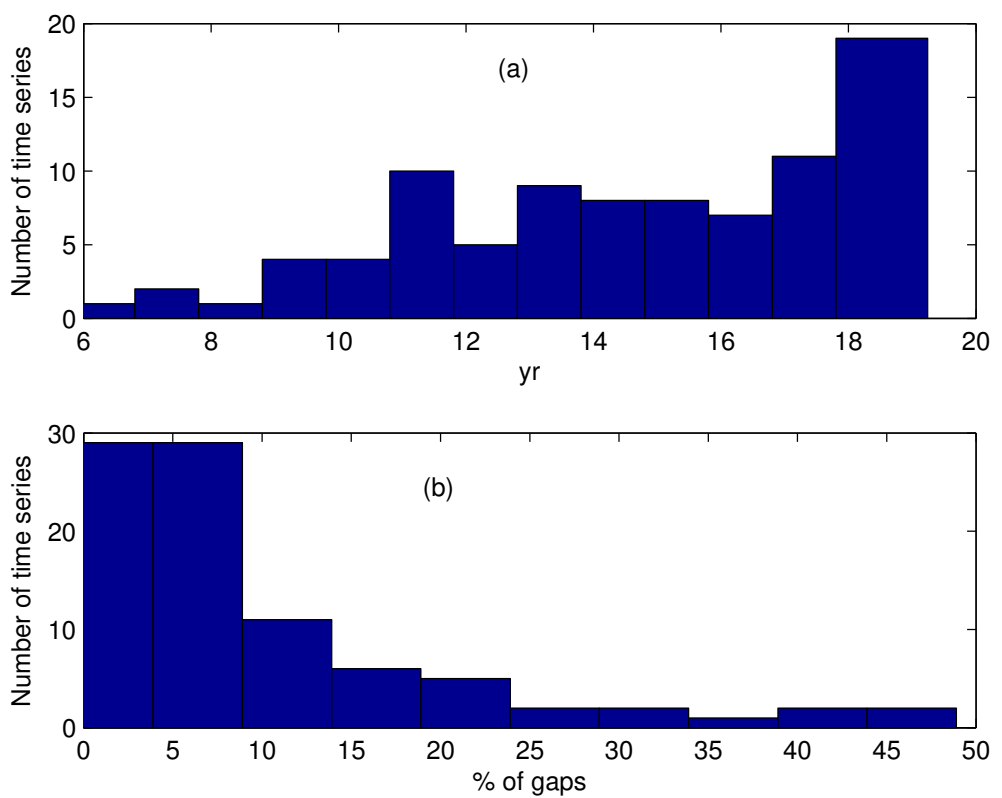

Fig. 12: Histograms for time series (JPL): Time series lengths (a) and \% of gaps (b).

Figs. 14d-f show the difference of MCMC and CATS estimating the white noise amplitude $\sigma_{w n}$. For some time series where the estimated $\sigma_{w n}$ is very small, CATS considers it to be null, i.e. all the noise is a pure power-law process. These are the points aligned at the vertical axis in all three Figs. 14d, 14e and 14f.

Sometimes when CATS sets $\sigma_{w n}$ to zero, it yields $N a N$ values for the uncertainties as it happens for the estimated East component velocity of THU3. The cause for this is a bad numerical behaviour of the computed Fisher matrix ${ }^{4}$. Another consequence of setting $\sigma_{w n}=0$ is that it shifts $-\kappa$ towards smaller values, thus diminishing the correlation within the noise and underestimating the uncertainties of all parameters of the model. Moreover, it makes $\sigma_{p l}$ larger, as the results for the synthetic data set suggested. As MCMC does not deal with derivatives these numerical issues are avoided, and it performs well even with a combination of power-law and white noise.

Finally, Figs. 15, 16, and 17, which correspond to the correlation between estimates for both methods, i.e. $v, y_{0}, A_{1 y r}^{c}, A_{1 y r}^{s}, A_{0.5 y r}^{c}$ and $A_{0.5 y r}^{s}$, respectively, show that both methods are in good agreement.

As the aforementioned figures suggest, estimates from both methods seem to align linearly, hence the consideration of a linear least-squares fit. Table 7 summarises the fit of the points (after removing outliers beyond $3 \sigma$ ) showing the slope and the ordinate for each parameter and all three components.

${ }^{4}$ S.D.P. Williams, personal communication, 2013. 

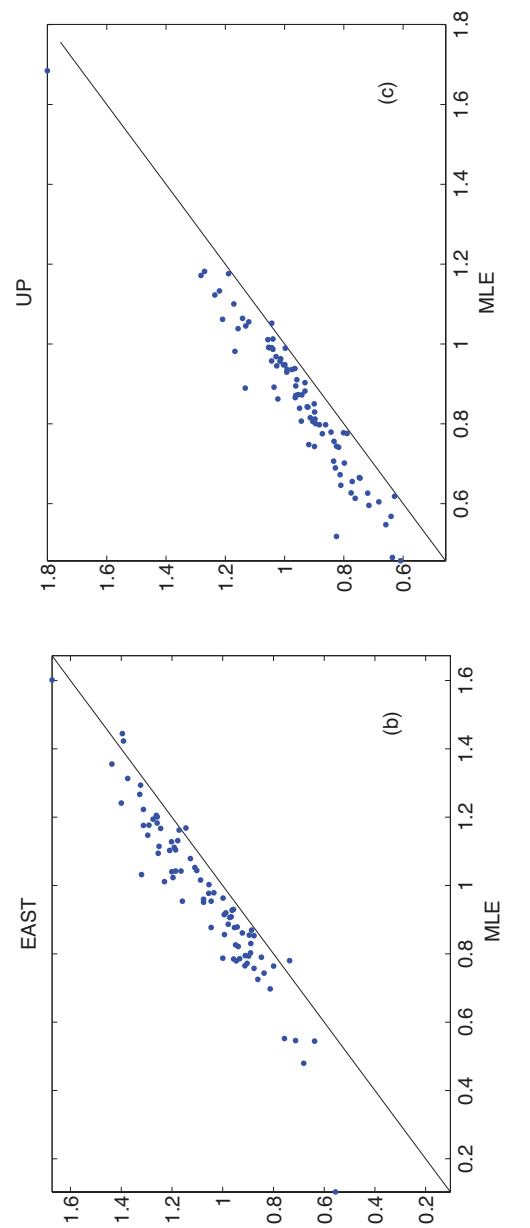

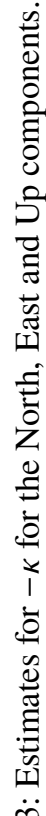

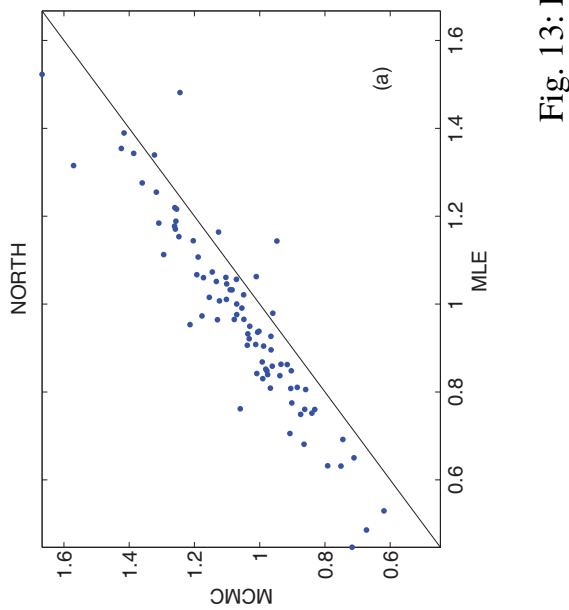




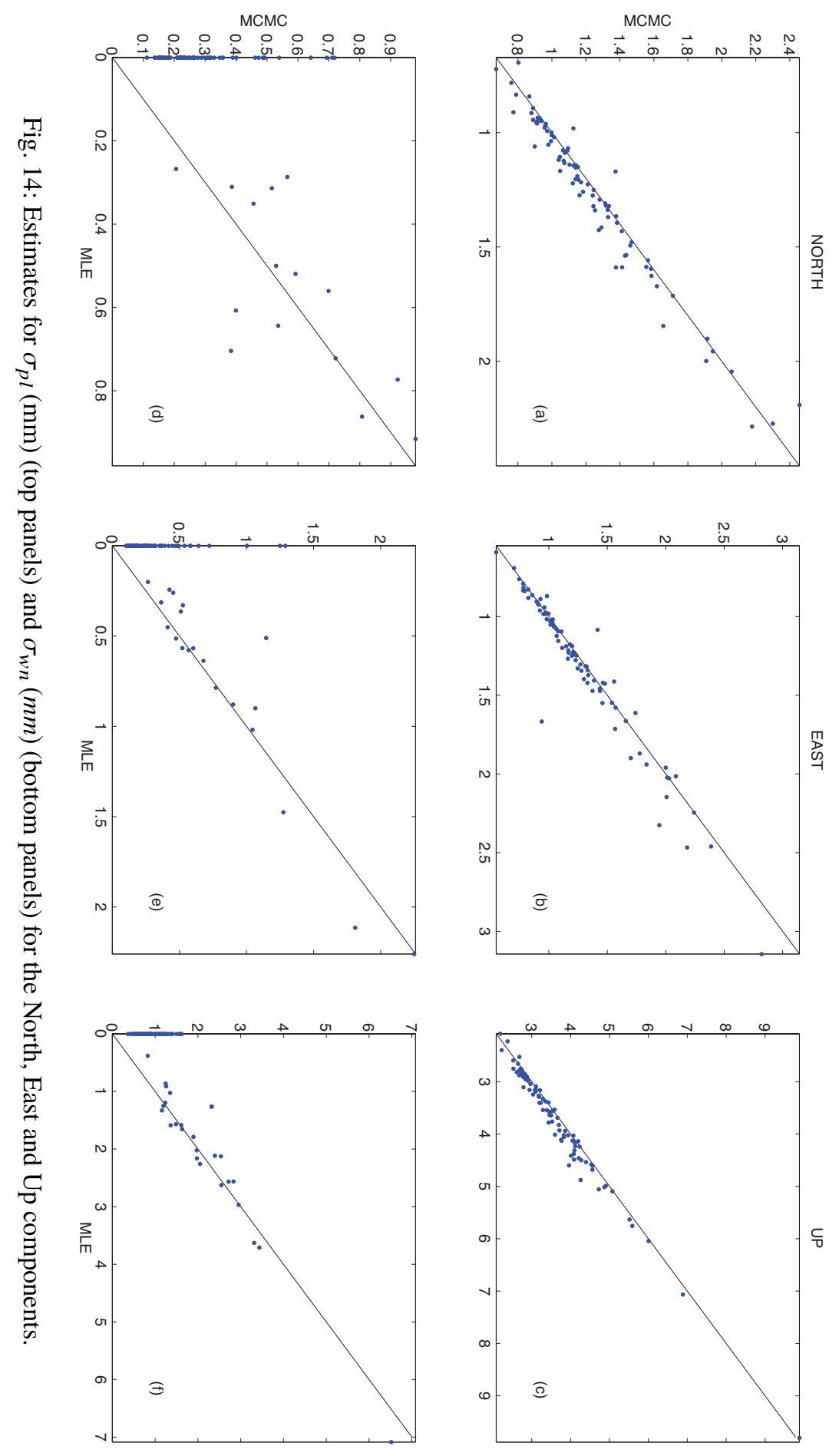



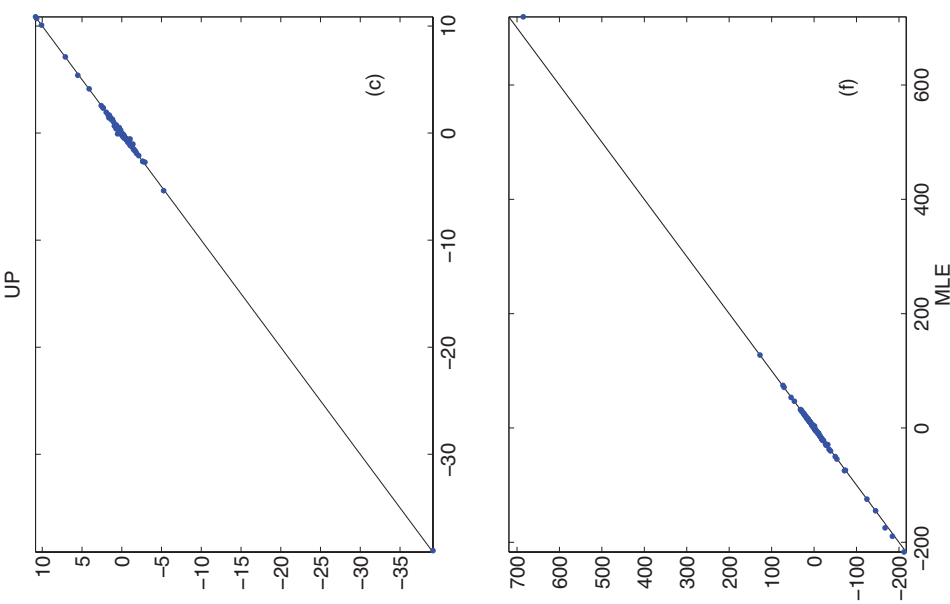

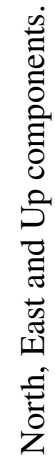
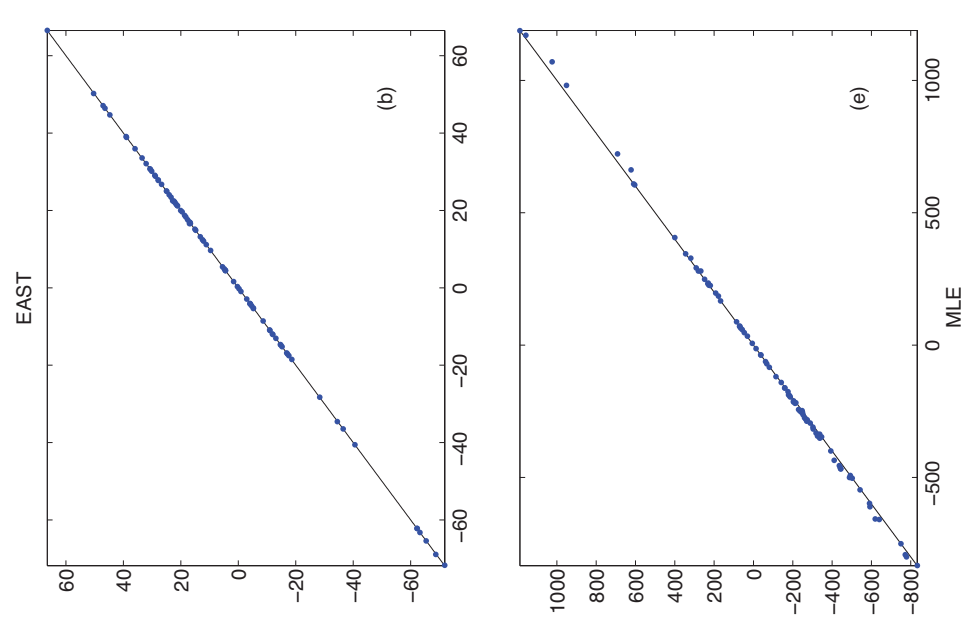

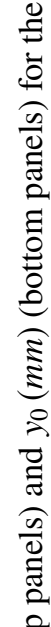
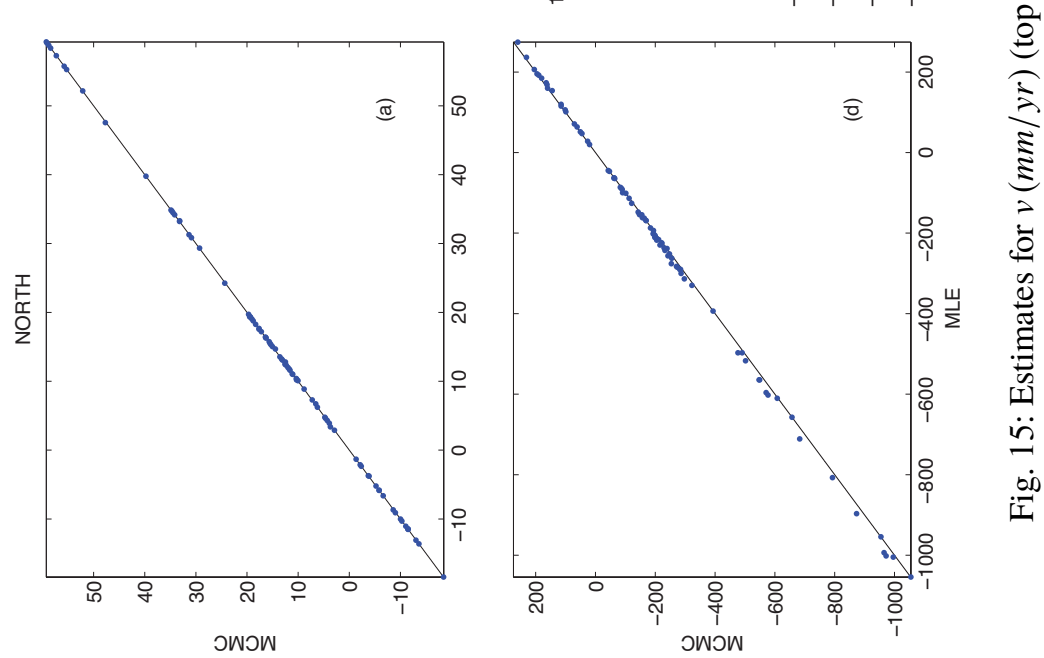


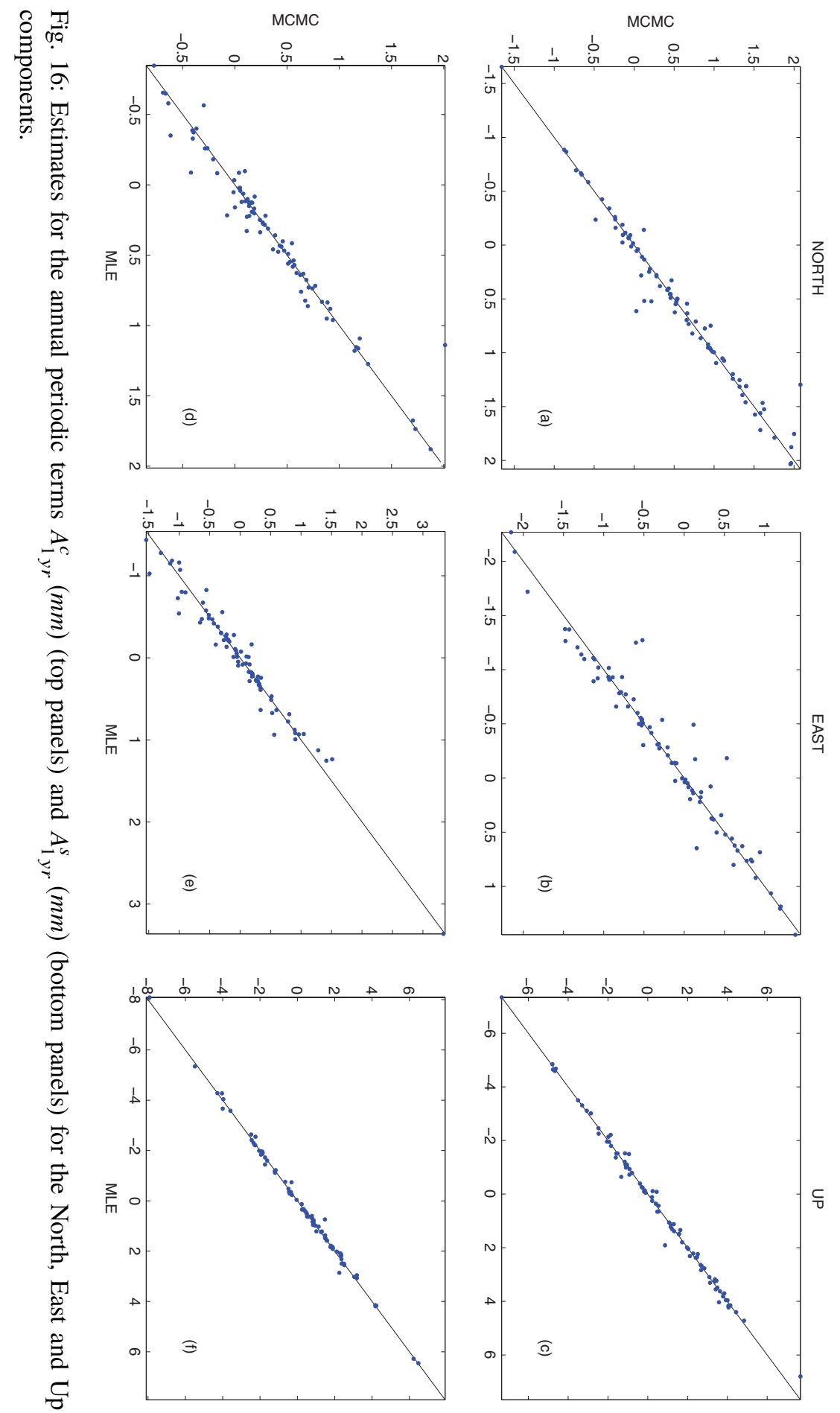



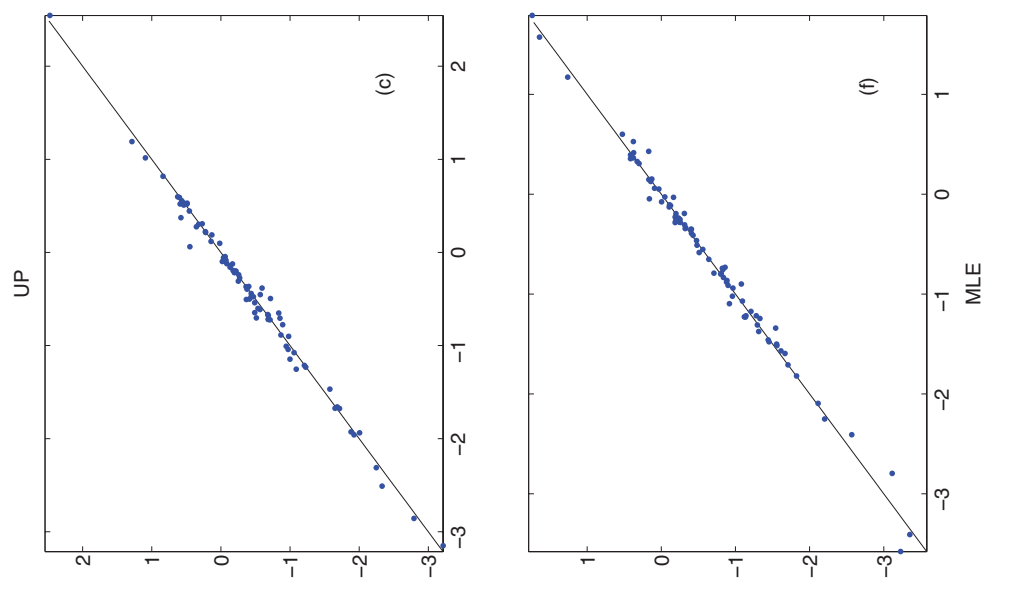

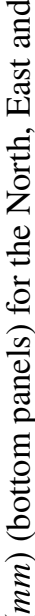
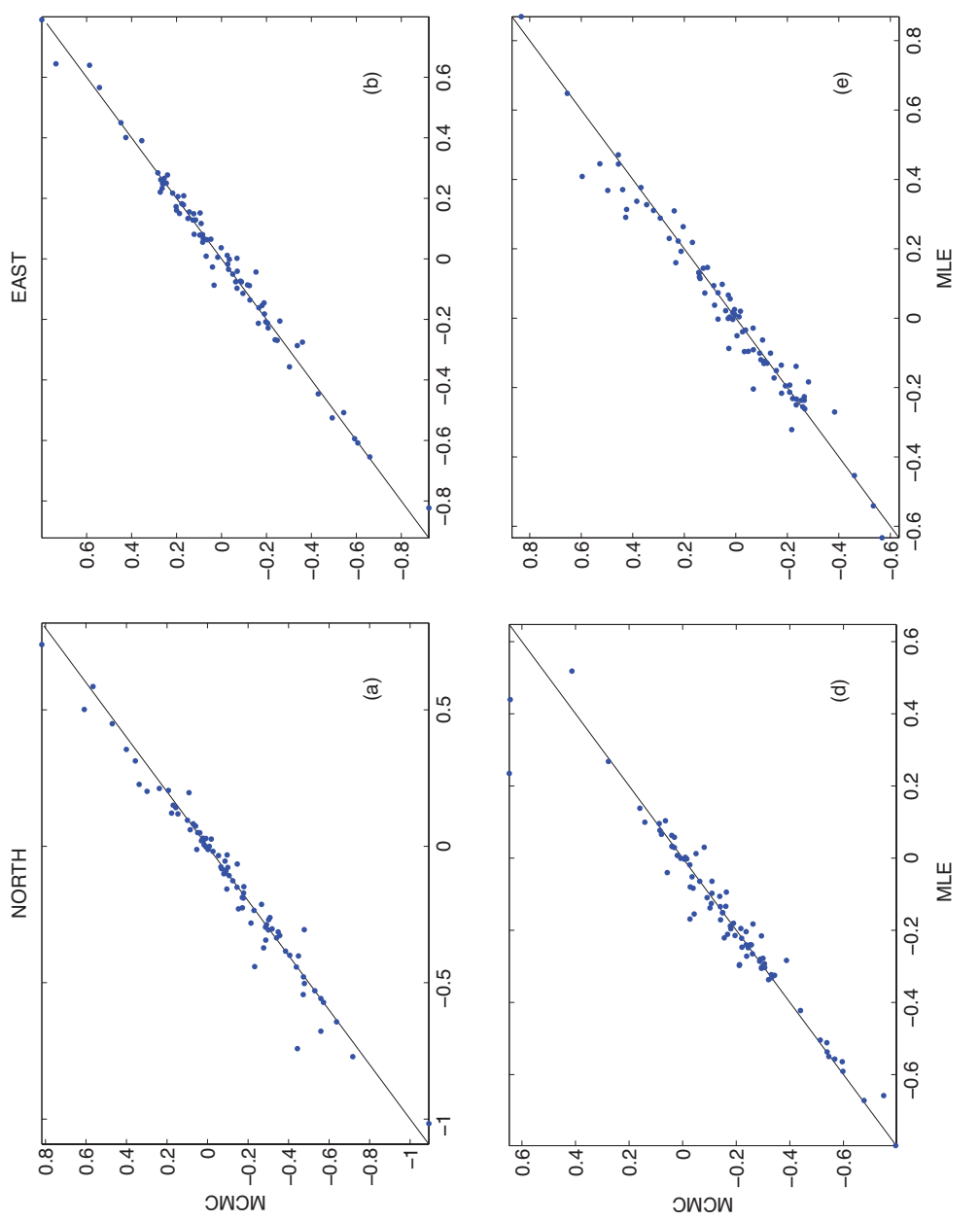

0
0
0
0
0

iุ 
Table 7: Parameter estimates for the LLS fit, as defined in Eq. 13, between MCMC and CATS results for the estimates parameters (JPL data).

\begin{tabular}{lllllll}
\hline \multirow{2}{*}{ Parameter } & \multicolumn{3}{c}{$\mathrm{N}$} & \multicolumn{2}{c}{$\mathrm{E}$} & \multicolumn{2}{c}{$\mathrm{U}$} \\
\cline { 2 - 7 } & \multicolumn{1}{c}{$a$} & \multicolumn{1}{c}{$b$} & $a$ & \multicolumn{1}{l}{$b$} & $a$ & \multicolumn{1}{l}{$b$} \\
\hline$-\kappa$ & 0.94 & 0.16 & 0.85 & 0.25 & 0.80 & 0.26 \\
$\sigma_{p l}(\mathrm{~mm})$ & 0.99 & -0.02 & 0.93 & 0.06 & 0.97 & -0.03 \\
$v(\mathrm{~mm} / \mathrm{yr})$ & 1.00 & 0.01 & 1.00 & 0.01 & 1.00 & 0.03 \\
$y_{0}(\mathrm{~mm})$ & 0.98 & 1.14 & 0.97 & 0.72 & 0.96 & -0.35 \\
$A_{1 y r}^{c}(\mathrm{~mm})$ & 1.04 & -0.01 & 0.98 & 0.01 & 1.00 & 0.01 \\
$A_{1 y r}^{s}(\mathrm{~mm})$ & 1.03 & -0.02 & 1.00 & -0.02 & 1.00 & 0.03 \\
$A_{0.5 y r}^{c}(\mathrm{~mm})$ & 0.97 & 0.01 & 1.02 & 0.00 & 0.99 & 0.01 \\
$A_{0.5 y r}^{s}(\mathrm{~mm})$ & 1.06 & 0.02 & 1.03 & 0.01 & 0.99 & 0.00 \\
\hline
\end{tabular}

The slopes for $v, \sigma_{p l}, A_{1 y r}^{c}, A_{1 y r}^{s}, A_{0.5 y r}^{c}$ and $A_{0.5 y r}^{s}$ are $a \sim 1$ which proves that both methods perform alike at estimating these parameters. Their ordinate values are at submillimetre level: $b \sim 10^{-2} \mathrm{~mm} / \mathrm{yr}$ and $b \sim 10^{-2} \mathrm{~mm}$, respectively.

The estimates of $y_{0}$, though $a \sim 1$, show differences at $\mathrm{mm}$ (North component in Table 7) and sub- $m m$ levels (East and Up components in Table 7).

The major differences are found among the $-\kappa$ estimates. The slopes for $-\kappa$ in all components are $a<1$, though the estimates from MCMC are larger. This is because at low values, the differences are larger. This was found in the analysis of the synthetic data set as well (see Figs. 6a and 7). Though it might be related to the way CATS estimates the white noise, in this case this was not possible to confirm because there were zero-white-noise values all along the diagonal of Figs. 13 and 14.

Uncertainties. The differences between both methods are shown in Fig. 18 for the uncertainties of the estimates of $v$ and $y_{0}$, and Figs. 19 and 20 for the uncertainties in the annual $\left(\sigma_{A_{1 y r}^{c}}\right.$ and $\left.\sigma_{A_{1 y r}^{s}}\right)$ and semi-annual $\left(\sigma_{A_{0.5 y r}^{c}}\right.$ and $\left.\sigma_{A_{0.5 y r}^{s}}\right)$ periodic terms, respectively.

The uncertainties of the estimated spectral index $-\kappa$ are not computed by the public code of CATS, therefore they are not shown here. Similarly, as CATS yields $\sigma_{\sigma_{w n}}=N a N$ when $\sigma_{w n}=0$ for some GPS time series, they are not plotted either.

In general, most of the uncertainties of $v$ and the periodic terms are larger for MCMC than for CATS. Figs. 18a-c, 19 and 20, show a linear correlation with most of the values from MCMC above the diagonal. Only $\sigma_{y_{0}}$ gets larger uncertainties for CATS than for the MCMC method, as Figs. 18d-f show.

Table 8 summarises the values for the parameters of the LLS fit. The variety of values for the slope $a$, indicates less agreement between the uncertainties of both methods than their respective parameters had. The slope ranges from $a=0.51$ for the North component of $\sigma_{y_{0}}$, up to $a=1.35$ (for the East component of $\sigma_{v}$ ).

The bias values range from $0.56 \mathrm{~mm}$ for the $y_{0}$ Up component to $0.01 \mathrm{~mm} / \mathrm{yr}$ for velocity East component. 

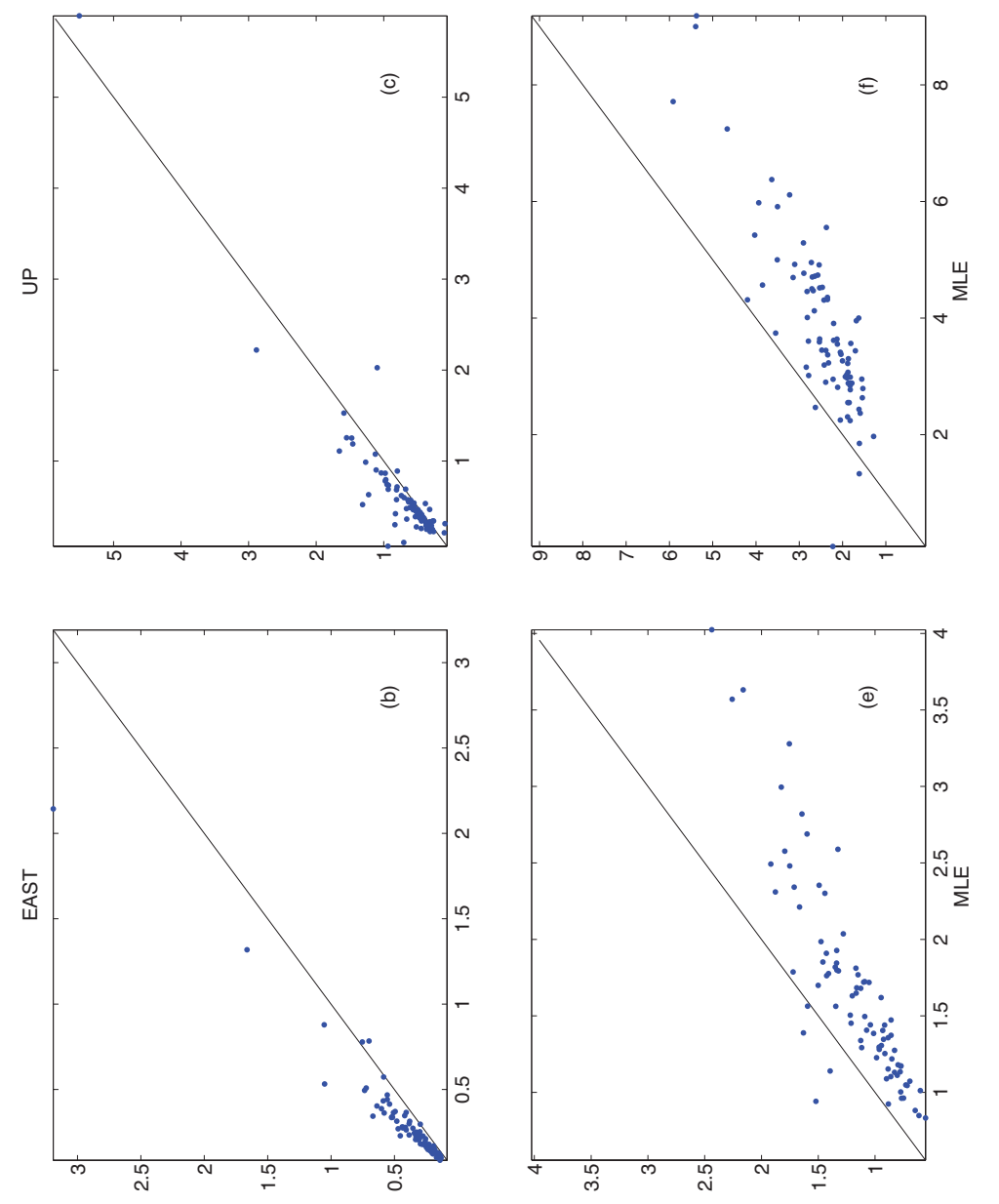

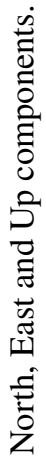
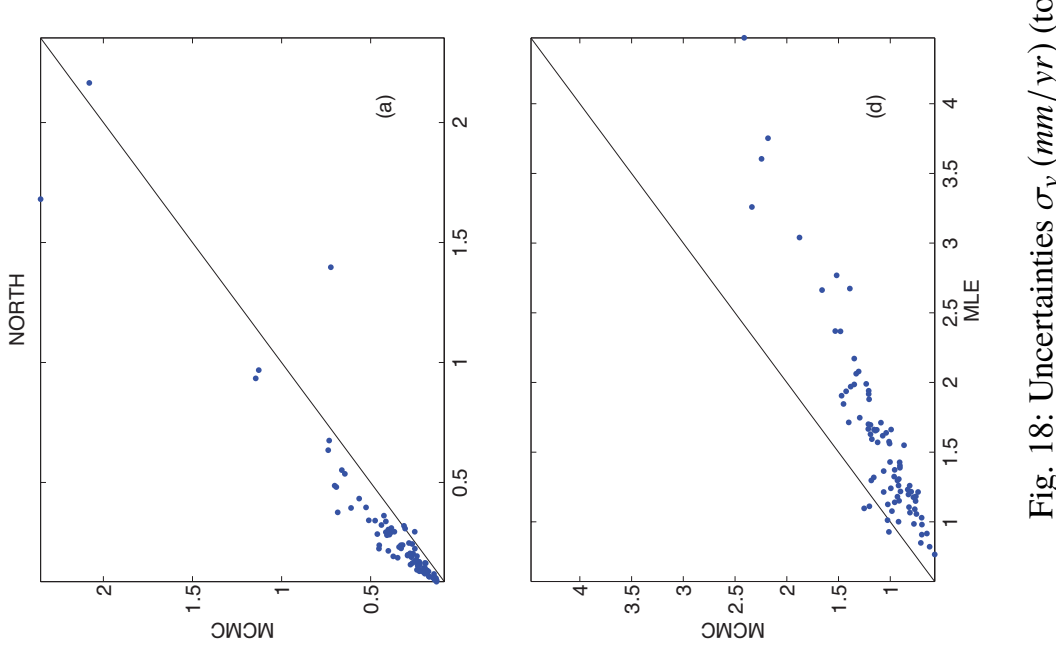


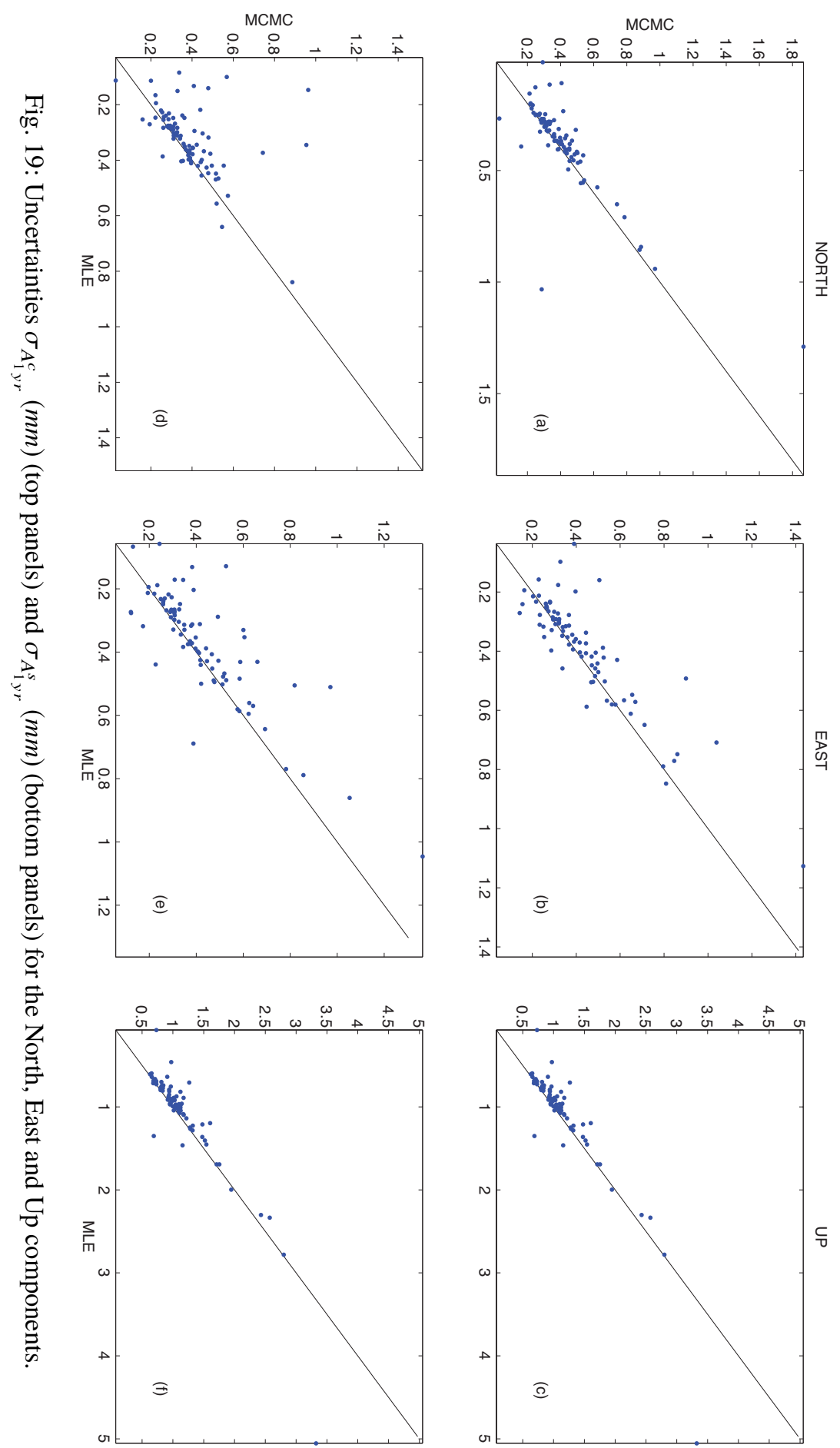



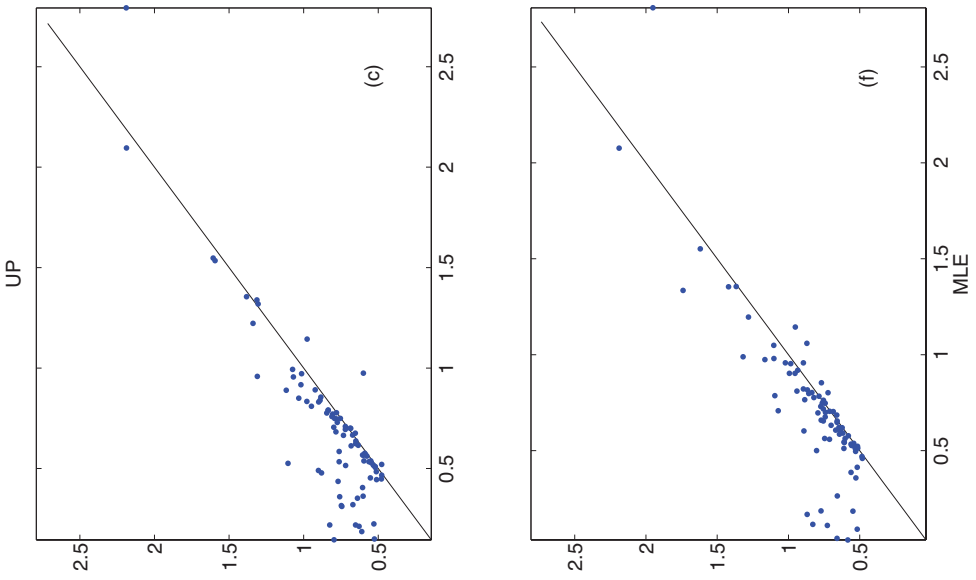

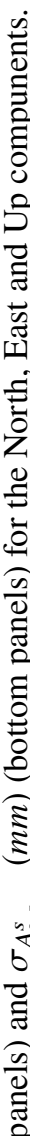
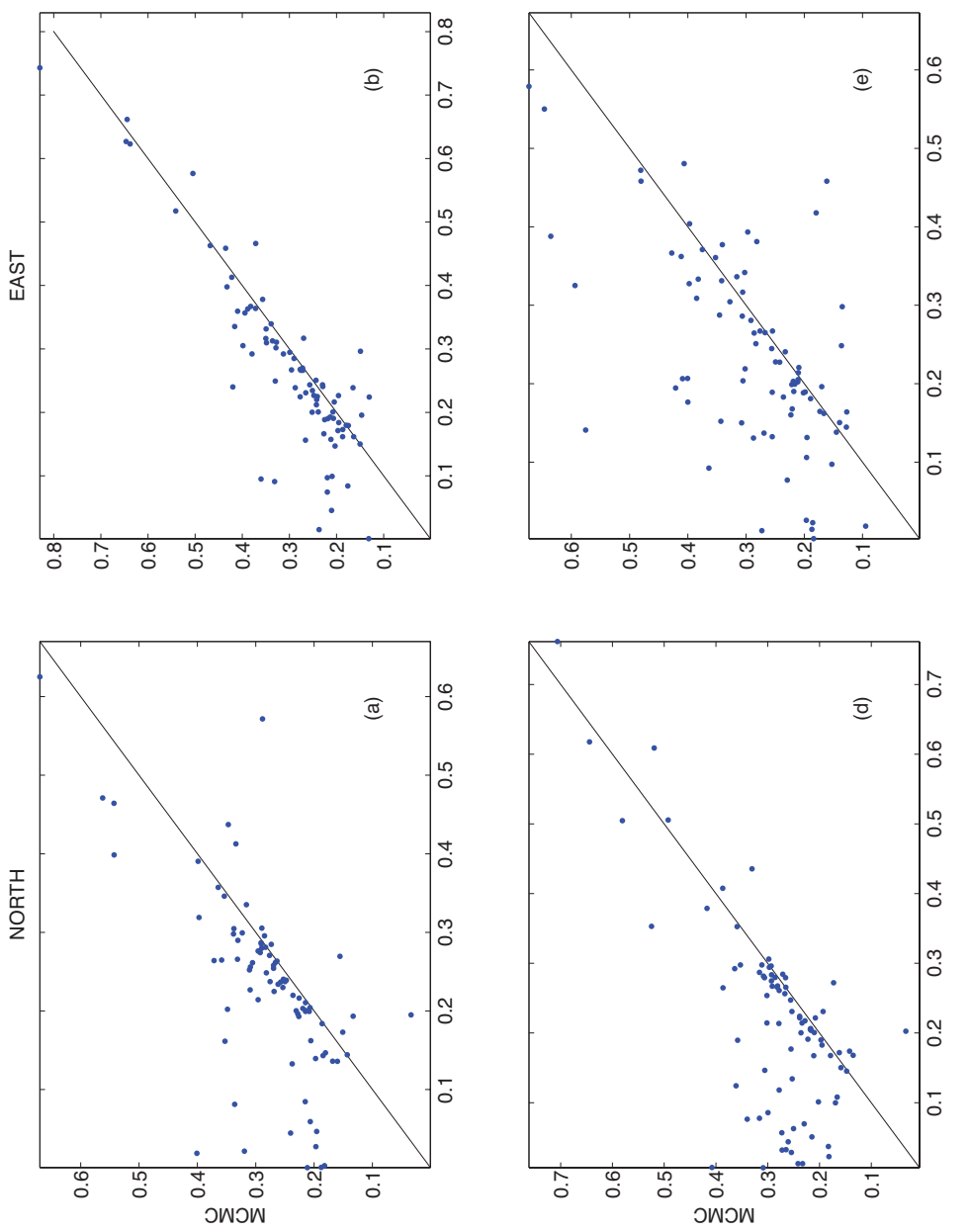

है 
Table 8: Parameter estimates of LLS fit, as defined in Eq. 13, for the MCMC and CATS results for the parameter uncertainties (JPL data).

\begin{tabular}{lllllll}
\hline Parameter & \multicolumn{3}{c}{$\mathrm{N}$} & \multicolumn{2}{l}{$\mathrm{E}$} & \multicolumn{2}{l}{$\mathrm{U}$} \\
\cline { 2 - 7 } & $a$ & $b$ & $a$ & $b$ & $a$ & $b$ \\
\hline$\sigma_{v}(\mathrm{~mm} / \mathrm{yr})$ & 1.00 & 0.09 & 1.35 & 0.01 & 0.93 & 0.16 \\
$\sigma_{y_{0}}(\mathrm{~mm})$ & 0.51 & 0.30 & 0.54 & 0.30 & 0.51 & 0.56 \\
$\sigma_{A_{1 y r}^{c}}(\mathrm{~mm})$ & 0.93 & 0.06 & 1.04 & 0.03 & 0.72 & 0.35 \\
$\sigma_{A_{1 y r}^{s}}(\mathrm{~mm})$ & 0.85 & 0.11 & 1.00 & 0.05 & 1.00 & 0.10 \\
$\sigma_{A_{0.5 y r}^{c}}(\mathrm{~mm})$ & 0.54 & 0.15 & 0.80 & 0.09 & 0.71 & 0.32 \\
$\sigma_{A_{0.5 y r}^{s}(\mathrm{~mm})}$ & 0.53 & 0.17 & 0.60 & 0.15 & 0.67 & 0.34 \\
\hline
\end{tabular}

Table 9: Values of the medians of the ratio $R_{p}$ (Eq. 14) and the difference $\Delta_{p}$ (Eq. 15)of the uncertainties (JPL data).

\begin{tabular}{lllllll}
\hline Parameter & \multicolumn{3}{c}{$\mathrm{N}$} & \multicolumn{2}{c}{$\mathrm{E}$} \\
\cline { 2 - 7 } & $R_{p}$ & \multicolumn{1}{c}{$\Delta_{p}$} & \multicolumn{1}{c}{$R_{p}$} & \multicolumn{1}{c}{$\Delta_{p}$} & \multicolumn{1}{c}{$R_{p}$} & \multicolumn{1}{c}{$\Delta_{p}$} \\
\hline$\sigma_{v}(\mathrm{~mm} / \mathrm{yr})$ & 1.40 & 0.08 & 1.40 & 0.08 & 1.18 & 0.08 \\
$\sigma_{y_{0}}(\mathrm{~mm})$ & 0.70 & -0.43 & 0.72 & -0.40 & 0.63 & -1.34 \\
$\sigma_{A_{1 y r}^{c}(\mathrm{~mm})}$ & 1.07 & 0.02 & 1.06 & 0.02 & 1.06 & 0.05 \\
$\sigma_{A_{1 y r}^{s}}(\mathrm{~mm})$ & 1.09 & 0.03 & 1.09 & 0.03 & 1.07 & 0.06 \\
$\sigma_{A_{0.5 y r}^{c}}(\mathrm{~mm})$ & 1.11 & 0.03 & 1.08 & 0.02 & 1.06 & 0.05 \\
$\sigma_{A_{0.5 y r}^{s}}(\mathrm{~mm})$ & 1.09 & 0.03 & 1.09 & 0.02 & 1.06 & 0.05 \\
\hline
\end{tabular}

Table 9 shows the median of the ratio and the difference of the uncertainties for the JPL data set. The values for the ratios are quite similar to those obtained for the synthetic time series (see Table 6), with $R_{v}=1.40$ being the biggest value (North and East components) and $R_{y_{0}}=0.63$ the smallest one (Up component). The periodic terms are also quite similar as their values range from 1.06 up to 1.11 . The most noticeable is that the median of the ratio of the velocities and periodic terms are larger than 1 for all three components, meaning that, unlike the results for $a$, the uncertainty estimates from MCMC are larger than those from CATS, namely $40 \%$ larger for the North and East components, and 18\% for the Up component, thus showing good agreement with the results for the synthetic data set.

The uncertainty of the estimated spectral index, $\sigma_{-\kappa}$, is not computed by CATS. This entails that CATS performs with one less parameter than the MCMC method, therefore, smaller uncertainties for the velocity estimates are expected from CATS. It is possible to check out this statement by setting $-\kappa$ as an input for both methods.

Therefore, it is reasonable to state that the main difference in the velocity uncertainties between both methods stems from the fact that CATS does not estimate the uncertainty of the spectral index, and, by doing so, the velocity uncertainties for all components are underestimated. 
On the other hand, CATS offers a quicker method than MCMC to estimate the model parameters and their uncertainties: CATS is around one order of magnitude faster than the MCMC method, therefore, if the difference in these uncertainties is not measurable, i.e. (so far) at sub-millimetre level, CATS is more time-efficient than MCMC.

Another argument in favour of the spectral index estimate (and its uncertainty) is provided by the Bayesian Information Criterion (BIC) (Schwarz, 1978). This criterion states the following: Given two models with different amount of parameters to estimate, the BIC favours the one with the largest maximum likelihood estimate $\left(L_{\max }\right)$ and penalises the amount of parameters $k$, or, equivalently:

$$
B I C=-2 \log \left(L_{\max }\right)+k \ln (N)
$$

where $N$ is the amount of data. According to Eq. 16, the smaller the BIC value, the better the model.

The BIC parameter is computed using the same method (firstly CATS, then MCMC) on the JPL data set for two models: One that estimates $-\kappa$, and another one that consider the spectral index as an input, namely, Flicker noise, i.e. $-\kappa=1$. Table 10 summarises the results for $\triangle B I C=B I C_{p l}-B I C_{\text {Flicker }}$, i.e. the difference between the BIC value from the power-law model and that from the Flicker-noise model. The second column shows that for 5\% of the stations, in the North and East components the power-law model provides a smaller BIC than the Flickernoise model. For the Up component $7 \%$ of the stations are better modelled with Flicker noise, whereas for $30 \%$ of the stations both models are equivalent, i.e. $-2<\Delta B I C<0$. A positive evidence, i.e. $-6<\Delta B I C<-2$, (third column) is shown for 54\% of the stations in the North component, and 55\% of them in the East component. Again, the Up component shows a smaller percentage of $45 \%$. Finally, a strong evidence is found in the fourth column of Table 10 for $41 \%, 40 \%$ and $28 \%$ of the stations for the North, East and Up components, respectively.

For the MCMC method it is assumed that the likelihood is Gaussian, then the mean and the maximum of the likelihood would be similar and the BIC criterion can be applied too. Table 11 shows the results for $\triangle B I C$ for the power-law model and the Flicker-noise model. For $2 \%$ and $1 \%$ of the stations in the North and East components, respectively, the power-law and the Flicker-noise model are considered equivalent $(-2<\triangle B I C<0$, second column). There is positive evidence for $7 \%, 4 \%$ and $4 \%$, and strong evidence for $80 \%, 82 \%$ and $81 \%$ of the stations for the North, East and Up components, respectively. The Flicker-noise model is better considered for $11 \%, 13 \%$ and $15 \%$ of the stations for the North, East and Up components. The results summarised in Tables 10 and 11 are in good agreement, denoting that for the majority of the stations there is positive evidence in favour of the power-law model for the MCMC and CATS methods.

To summarise:

- Both methods estimate parameters in good agreement as Tables 3 and 7 show. 
Table 10: Values of $\triangle B I C \equiv B I C_{p l}$ for the North, East and Up components for a power-law model and a Flicker-noise model using CATS (JPL data set).

\begin{tabular}{cccc}
\hline & $-2<\Delta B I C<0$ & $-6<\Delta B I C<-2$ & $\Delta B I C<-6$ \\
\hline $\mathrm{N}$ & $5 \%$ & $54 \%$ & $41 \%$ \\
$\mathrm{E}$ & $5 \%$ & $55 \%$ & $40 \%$ \\
$\mathrm{U}$ & $30 \%$ & $45 \%$ & $28 \%$ \\
\hline
\end{tabular}

Table 11: Values of $\triangle B I C \equiv B I C_{p l}$ for the North, East and Up components for a power-law model and a Flicker-noise model using MCMC (JPL data set).

\begin{tabular}{cccc}
\hline & $-2<\Delta B I C<0$ & $-6<\Delta B I C<-2$ & $\Delta B I C<-6$ \\
\hline $\mathrm{N}$ & $2 \%$ & $7 \%$ & $80 \%$ \\
$\mathrm{E}$ & $1 \%$ & $4 \%$ & $82 \%$ \\
$\mathrm{U}$ & $0 \%$ & $4 \%$ & $81 \%$ \\
\hline
\end{tabular}

- As the MCMC method simultaneously estimates all parameters, including the spectral index, it yields [1.18 - 1.40] times larger uncertainties for the model parameters than CATS (see Table 8).

- According to Tables 3, 7, and 6, 8, estimated parameters and their uncertainty ratios for real data sets show great consistency with those for the synthetic data.

- The BIC criterion denotes that the power-law model is better than the Flicker-noise model for most of the stations analysed with the MCMC and CATS methods.

- As a consequence of the BIC results, it is necessary to compute the spectral index estimate uncertainty in order to get more realistic uncertainties for all model parameters.

\subsubsection{Computational Time}

CATS computes the covariance matrix just once, and its computation is the most memory-demanding computational process, thus slowing down the estimation process. On the other hand, the MCMC method computes the covariance matrix for each value of $\alpha$ within the Markov chain. Therefore, the MCMC method requires more computational time. Indeed, Fig. 21 shows the difference between the CPU time needed for the MCMC (red points) and the CATS (blue points) methods. These are CPU times for each of GPS position time series from the JPL data set.

Both methods scale with the number of epochs as $N^{-\kappa}$, where $-\kappa=2.5$ for MCMC, and $-\kappa=2.8$ for CATS. CATS is around one order of magnitude faster than the MCMC method. Therefore, if the difference in these uncertainties is not measurable (at sub-millimetre level), CATS would be more time-efficient than the MCMC method. 


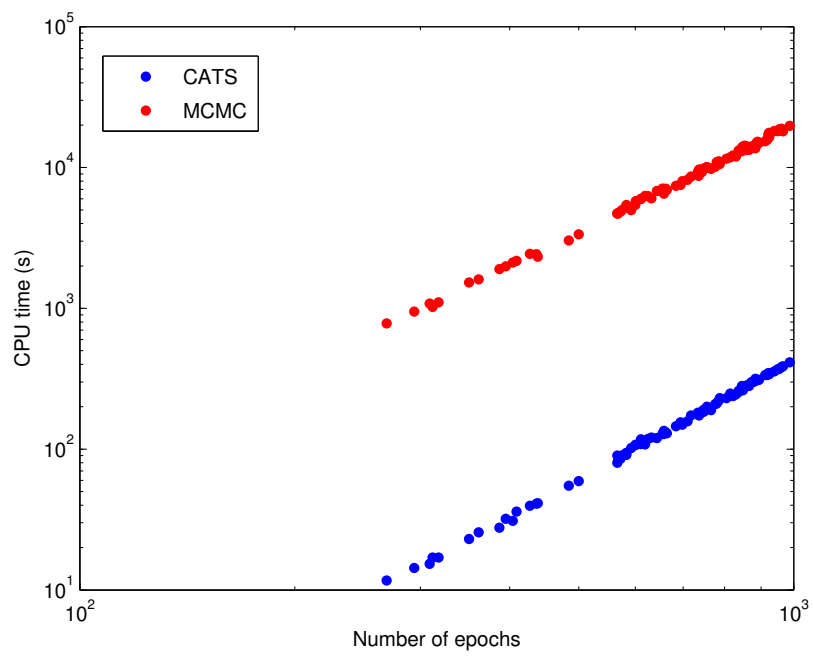

Fig. 21: Computational time of the estimation of parameters with the MCMC method (red) and CATS (blue) for the JPL data set.

With the development of faster implementations of the MLE method in more recent versions of CATS or the Hector software (Bos et al., 2012), the computational time is reduced even further than when we carried out this analysis. It is apparent that the time requirement for the MCMC method in its current implementation would be prohibitive for many applications.

\subsection{Plate Motion Models}

The analysis with MCMC and CATS carried out on the JPL position time series was repeated on 171 GPS stations from JPL in order to estimate an absolute plate motion model (PMM) for each method (MCMC-PMM and CATS-PMM for the MCMC and CATS methods, respectively), thus assessing the performance of both methods and how their differences would affect the constraints on plate motion models.

A comprehensive analysis on plate motion is beyond the scope of this chapter, and the following subsections just show how the differences of these two statistical methods lead to different constraints on any geophysical model, e.g. plate motion. 


\subsubsection{Station selection}

Following Altamimi et al. (2012) for the station selection criteria only those stations far away from the plate boundaries and without significant glacial isostatic adjustment were used. Listed in the second column in Tables 15 and 16 in Appendix A, 171 stations were used to estimate the PMM for each method.

Unlike Altamimi et al. (2012), where GPS, SLR, DORIS and VLBI techniques were considered, only GPS stations were analysed herein, therefore some differences are expected with respect to their results. Another consequence of not using the same techniques is that the number of stations is different to Altamimi et al. (2012), wherein 206 sites were analysed. Therefore small differences in the derived plate motion models are expected.

\subsubsection{Plate Motion Model Results}

Station velocities and their $2 \sigma$ uncertainties (black ellipses showing $95 \%$ confidence level) from both methods are shown in Fig. 22. Both methods are in good agreement with the North American plate moving westwards and Eurasia moving eastwards. These two plates contain around $57 \%$ of sites. The Nubia and Somalia plates jointly move north-eastwards. The South American plate has eight sites moving northwards and Antartica shows more stability than the other plates, though the directions of the vector velocities are more varied. The largest velocities are those on the Australian (moving north-eastwards) and the Pacific (moving north-westwards) plates.

Tables 12 and 13 summarise the results for the PMM from the MCMC and CATS methods, respectively. The first column in both Tables stands for the code of the plates as it follows: AMUR for Amurian; ANTA for Antartica; ARAB for Arabia; AUST for Australia; CARB for Caribbean; EURA for Eurasia; INDI for India; NAZC for Nazca; NOAM for North America; NUBI for Nubia; PCFC for Pacific; SOAM for South America; SOMA for Somalia, and SUND for Sunda.

The second column shows the number of stations $(N S)$ on each plate, whereas the next three columns summarise the results for angular velocities in the three directions of the coordinate axes $\omega_{x}, \omega_{y}$ and $\omega_{z}$.

The Euler pole components are in the sixth and seventh columns, respectively, with the Euler pole angular velocity in the eighth column. Finally, the last two columns summarise the weighted root mean square (WRMS) of the residuals for each plate. The last line shows the global WRMS of the PMM considered for each method.

Concerning the global WRMS, CATS-PMM gives $0.72 \mathrm{~mm} / \mathrm{yr}$ and $0.80 \mathrm{~mm} / \mathrm{yr}$ for the North and East components, respectively; whereas MCMC-PMM gives $0.73 \mathrm{~mm} / \mathrm{yr}$ and $0.76 \mathrm{~mm} / \mathrm{yr}$ for the North and East components, respectively.

Taking into account all nine parameters involved, the RMS computed for the MCMC is usually smaller than that for CATS as Fig. 23 shows for all components. 

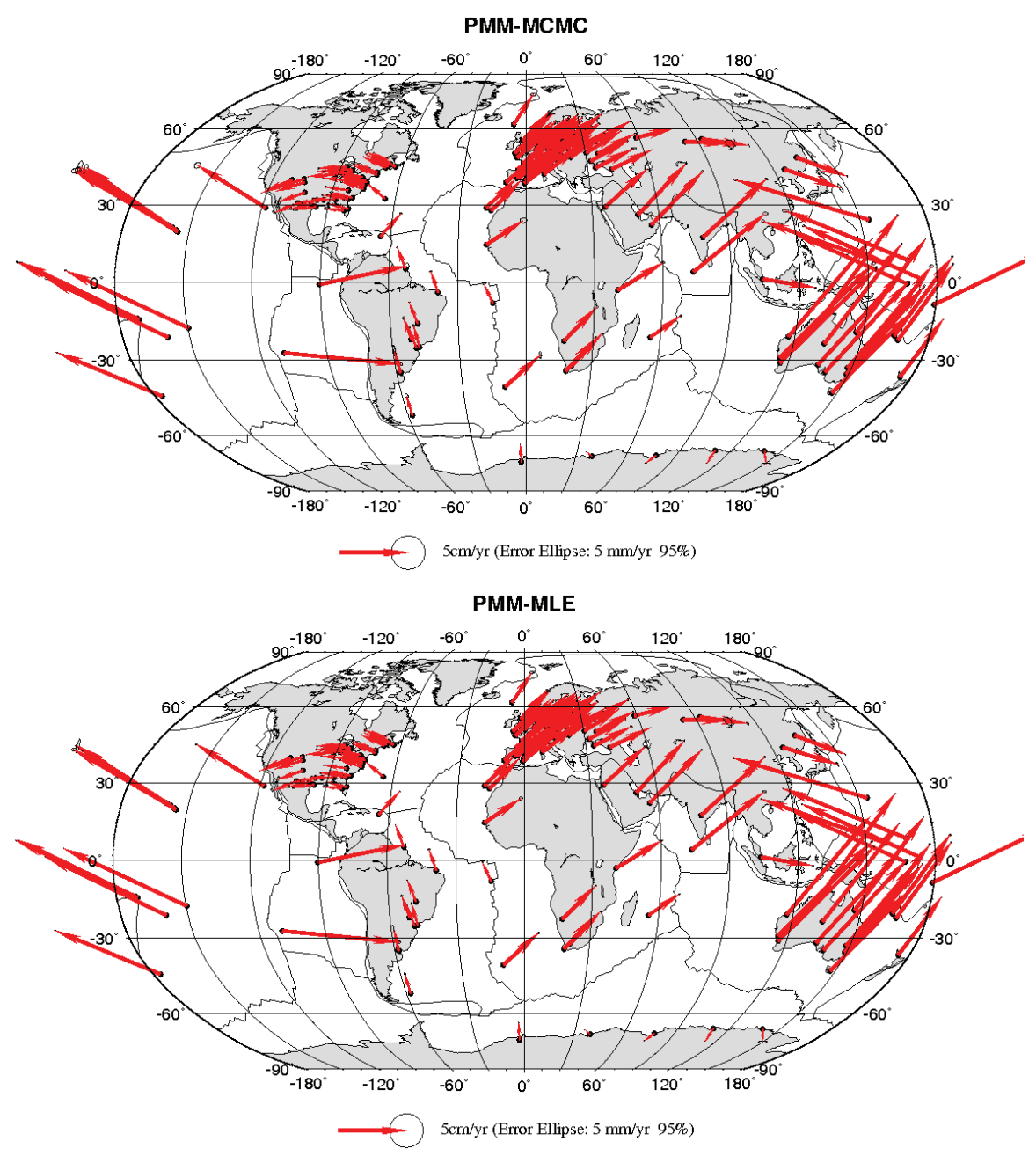

Fig. 22: Site velocities from the MCMC (top panel) and CATS (bottom panel) methods.

In this figure, the differences between the RMS for the MCMC and the CATS methods for all three components are shown. Systematically, for most of the stations analysed, MCMC provides slightly better estimated parameters, though the differences for the North and East components are at sub-millimetre level. As for the Up components, there is almost no difference, as the histogram is centred around $0 \mathrm{~mm}$ with $\sim 90 \%$ of the stations being in the range from $-0.002 \mathrm{~mm}$ to $0.002 \mathrm{~mm}$.

In general, the cartesian components of the angular velocities ( $3^{\text {rd }}-5^{\text {th }}$ columns) for both methods are similar. Angular velocities for AMUR, ANTA, AUST, CARB, INDI, NAZC, NUBI, SOAM and SOMA plates show good agreement at $1 \sigma$ confi- 
dence level. For EURA only $\omega_{y}$ from both methods agree at $1 \sigma$, whereas for SUND $\omega_{x}$ and $\omega_{y}$ agree at the $1 \sigma$ confidence level. Only for three plates, ARAB, NOAM and PCFC, the estimated angular velocities disagree at $1 \sigma$.

In general, the uncertainties for the MCMC method are larger than for CATS. This is consistent with previous results concerning the linear velocities obtained for the synthetic and JPL time series. There are though, some exceptions, namely for the AUST, EURA (all three components) and SUND ( $\mathrm{x}$ and $\mathrm{z}$ components) plates. These three plates also showed disagreements at the $1 \sigma$ confidence level concerning their angular velocities.

As for the Euler poles, all the previous plates which were in good agreement for the angular velocities at the $1 \sigma$ confidence level, show the same agreement for the Euler pole coordinates and angular velocities, except for the SUND plate. Concerning ARAB, NOAM and PCFC, once more, they do not agree at $1 \sigma$ confidence level.

Results for the angular velocity from the MCMC method are in good agreement with the ITRF2008-PMM from Altamimi et al. (2012) (see Tab. 3 therein). All three components of the angular velocities for the following plates are consistent with each other at the $2 \sigma$ (95\%) confidence level: AMUR, ANTA, ARAB, AUST, NAZC, NOAM, NUBI, PCFC, SOAM and SOMA. For the other plates, i.e. CARB, EURA, INDI and SUND, at least two out of three components showed good agreement at the $2 \sigma$ confidence level.

The CATS-PMM showed larger differences than the MCMC-PMM with the ITRF2008-PMM: only results for AMUR, ANTA, AUST, NAZC and SOAM agreed at the $2 \sigma$ confidence level. The uncertainties of MCMC-PMM, CATS-PMM and ITRF2008-PMM were at the same level of magnitude but those from the former two methods showed more discrepancies with those from ITRF2008-PMM. The reason for this is that ITRF2008 stems from the composition of different geodetic techniques and uses a different number of sites.

In general, the reduced chi-square $\chi_{\text {red }}^{2}=r^{\prime} \mathbf{C}_{\mathbf{v v}} r / f$ is computed as a tool to compare models, where $f=N S-N P$ is the number of degrees of freedom, with $N P$ being the number of plates.

For MCMC, $\chi_{\text {red }}^{2} \sim 501$; whereas for CATS, $\chi_{\text {red }}^{2} \sim 802$. The best model should be that closer to the ideal value, i.e. $\chi_{\text {red }}^{2}=1$, thus each degree of freedom would contribute with the same amount of uncertainty. In order to get a better model, i.e. with more realistic uncertainties, the covariance matrix is rescaled in such a way that $\chi_{\text {red }}^{2}=1$. Considering the $\chi^{2}$ values above for each model, uncertainties from the MCMC method should be 22.4 times larger, and 28.3 times larger for the CATS method. This would suggest that the uncertainties from the MCMC method are less underestimated than those from CATS. The ratio of these two scale factors is $\sim 1.30$, which is consistent with the ratio of the uncertainties for the estimated velocities from the synthetic and JPL data sets. 


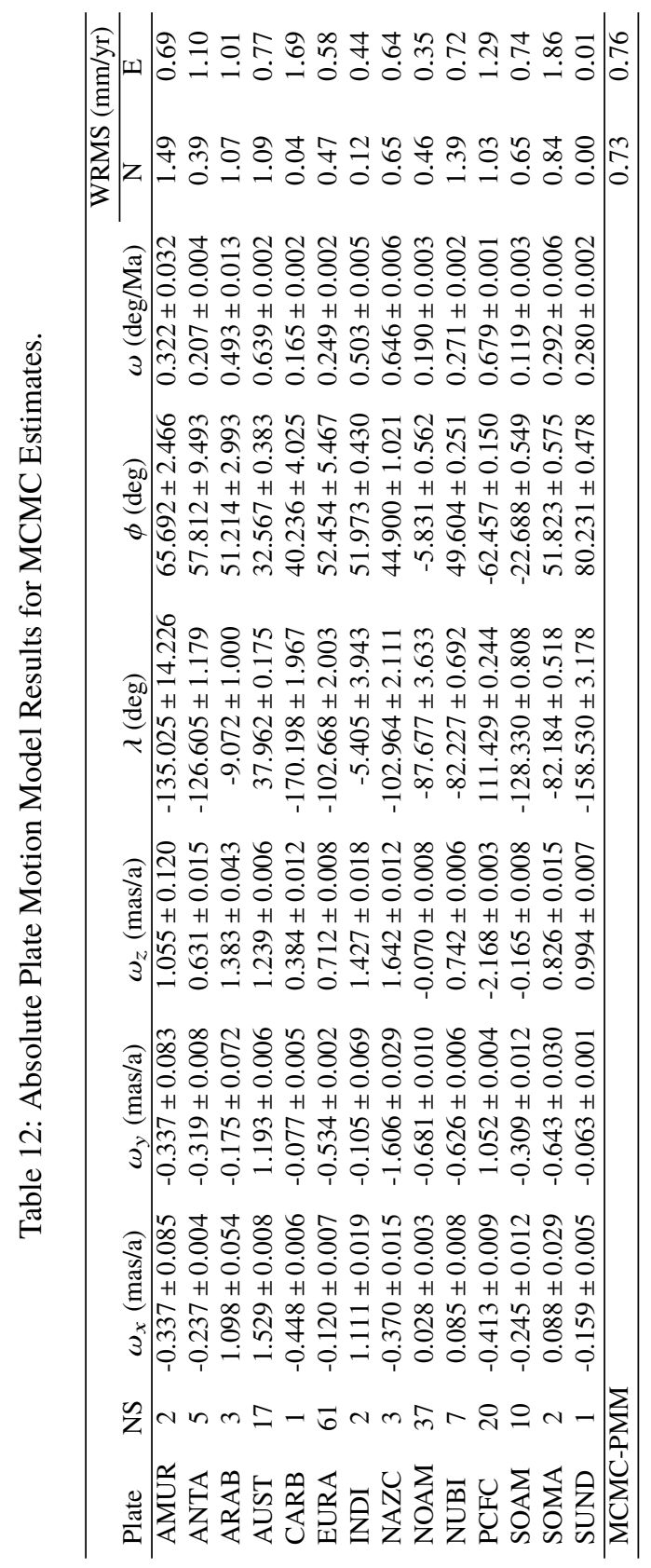




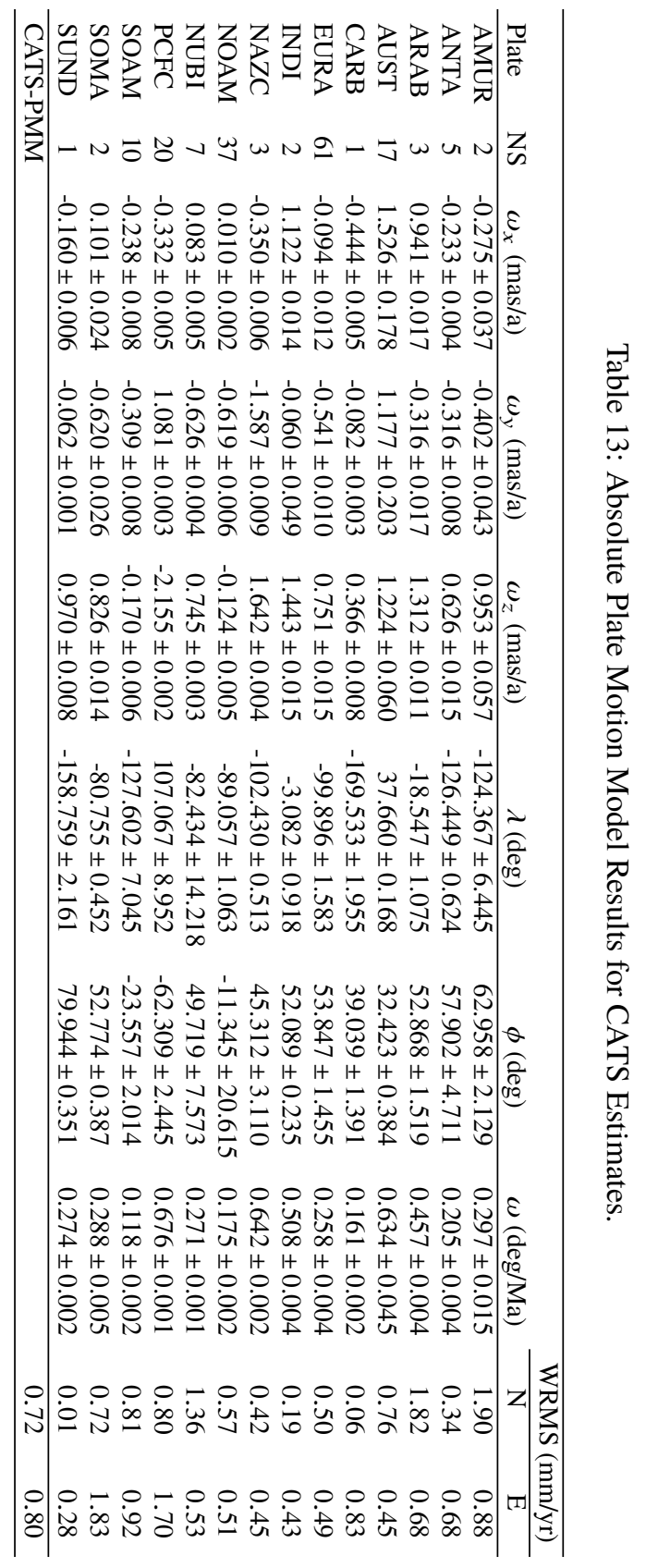



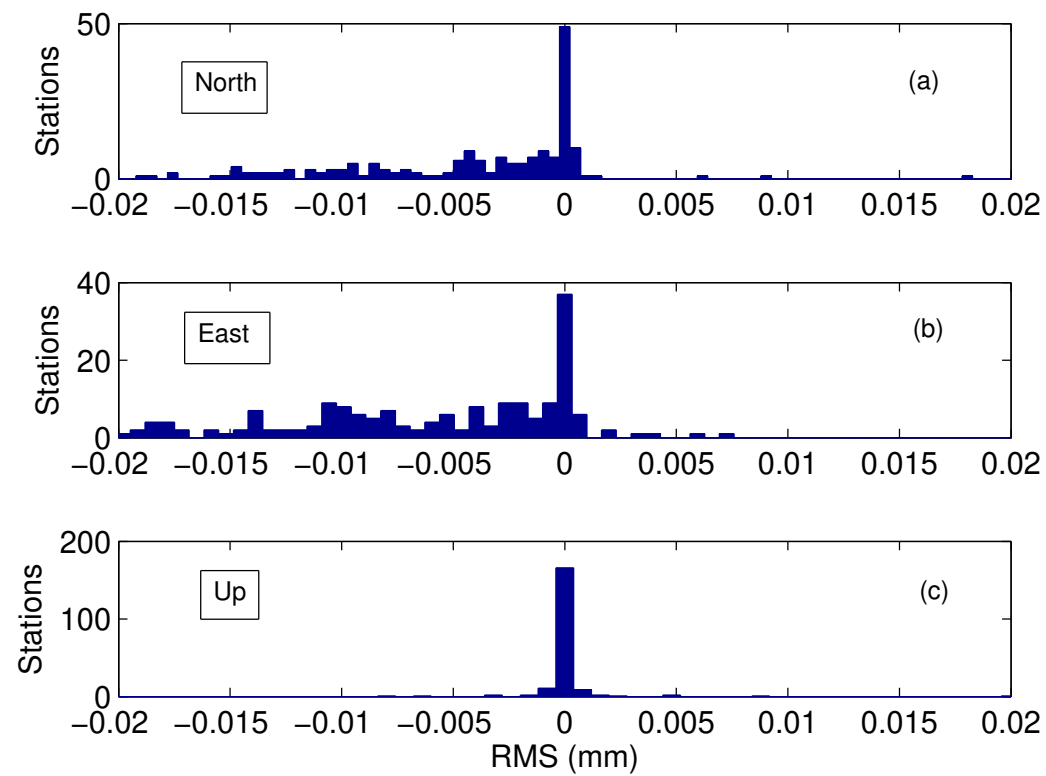

Fig. 23: Differences in $\mathrm{mm}$ between RMS for MCMC and CATS methods for all three components.

\subsection{Gravity Time Series}

Superconducting gravimeter data are measurements of the local relative variations of the gravity field. These variations are derived from vertical displacements of a hollow superconducting sphere that levitates in a persistent magnetic field (Goodkind, 1999). The gravity measurements at Membach, Belgium, provided by Olivier Francis and Michel van Camp, are shown in Fig. 24. The time series of the drift-corrected data spans from August 1995 until October 2011. For further details concerning the measurements, please see Van Camp et al. (2005).

The trend of the time series provides information about changes in the gravity field due to mass displacements, e.g. hydrological flows, and vertical displacements. This time series is a good example of highly time-correlated noise and its influence on estimating the trend and its uncertainty. Compared to the position time series from GPS the variability in the gravity series relative to the magnitude of the trend is significantly different. Therefore it provides an independent data set to evaluate the MCMC method. 


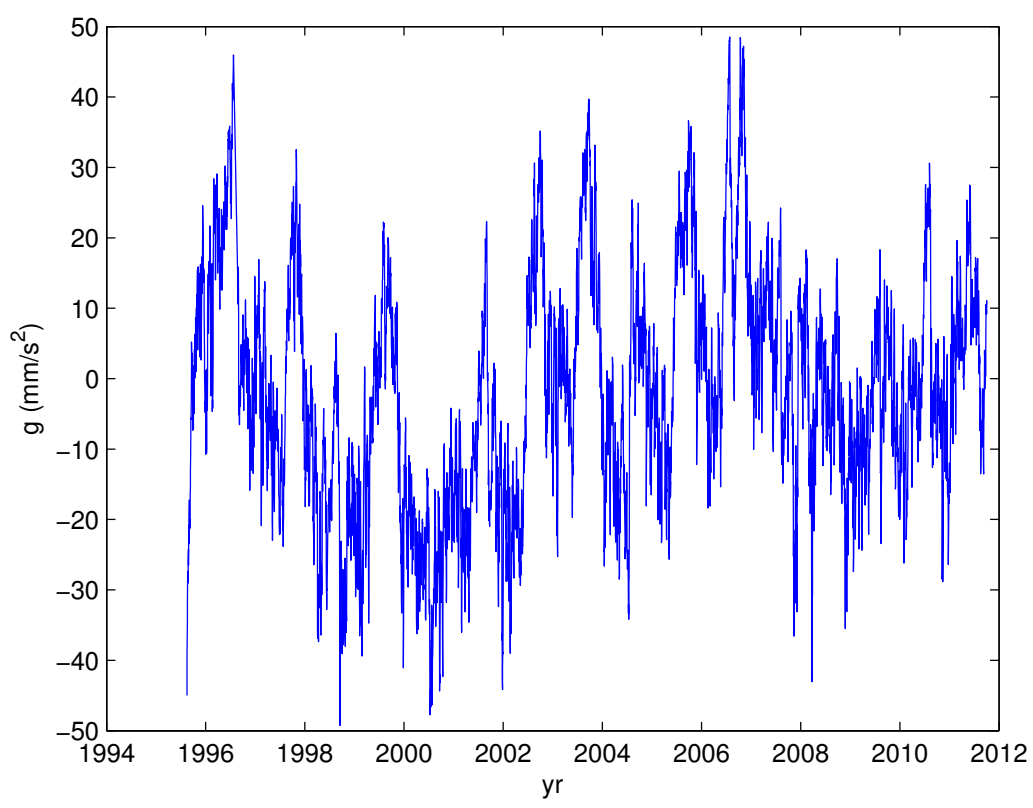

Fig. 24: Gravity field measurements at Membach.

Table 14: Parameter estimates and uncertainties for the superconducting gravity measurements at Membach station, Belgium.

\begin{tabular}{cccc}
\hline$-\kappa$ & $\sigma_{p l}\left(\mathrm{~nm} / \mathrm{s}^{2}\right)$ & $v\left(\mathrm{~nm} / \mathrm{s}^{2} / \mathrm{yr}\right)$ & $y_{0}\left(\mathrm{~nm} / \mathrm{s}^{2}\right)$ \\
\hline $2.24 \pm 0.02$ & $3.40 \pm 0.02$ & $0.81 \pm 0.12$ & $3.72_{-0.68}^{+0.64}$ \\
\hline
\end{tabular}

The analysis performed with MCMC yielded the results summarised in Table 14. The model assumed was a linear combination of linear trend plus a time-correlated noise process.

The first thing to note is the high value of the spectral index, $-\kappa=2.24$. This clearly indicates that the gravity time series contains a non-stationary process. A similar result $(-\kappa=2.4)$ was already obtained from a shorter sample of the time series which spanned to 2004 (Van Camp et al., 2005). It is a Random-Walk process, meaning that the gravity field is randomly evolving in time due to stochastic changes of mass distribution.

Fig. 25 shows the histogram of the estimated parameters. Figs. 25a and 25b show that $-\kappa$ and $\sigma_{p l}$, respectively, have Gaussian distributions.

The velocity although it does not seem Gaussian has an absolute maximum.

On the other hand, the ordinate $y_{0}$ is multimodal. This is typical of non-stationary stochastic processes, where the noise adds some velocity into the trend. This feature 

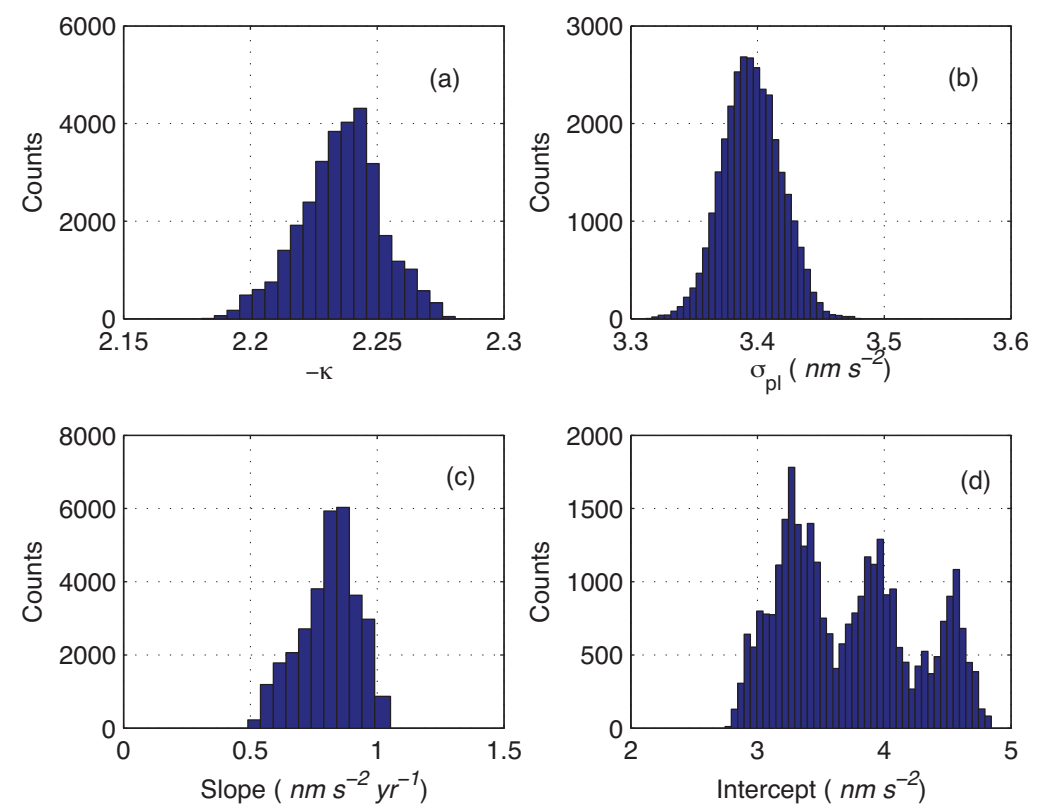

Fig. 25: Histograms for $-\kappa, \sigma_{p l}, v$ and $y_{0}$.

could be difficult to detect with an optimisation method as, for example, MLE as implemented in CATS, as it could have ended up at any of the maxima, and not necessarily at the absolute one.

The MCMC analysis of the time series of the superconducting gravity measurements has shown another advantage of using an integrator method such as MCMC rather than an optimisation method such as MLE. Due to the characteristics of the algorithm, it explores the surrounding areas of a maximum, thereby spotting other local maxima. This is the case for the ordinate parameter $y_{0}$ (see Fig. 25d). This is typical of non-stationary stochastic processes, where the noise adds some velocity into the trend. Therefore, it is advisable to use the MCMC method for time series with high spectral index, e.g. $-\kappa \sim 2$.

\subsection{Mean Sea Level Time Series}

The third real data set to be analysed with the MCMC method were the monthly mean sea level (MSL) records from the Revised Local Reference (RLR) data base 

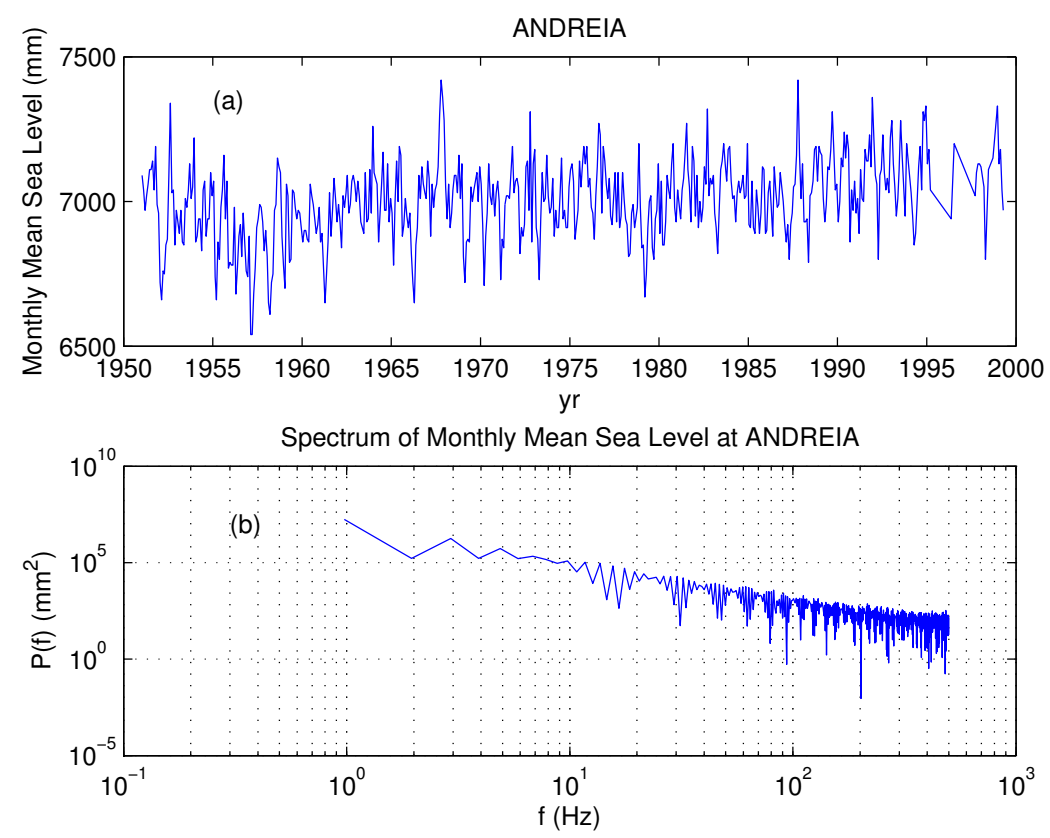

Fig. 26: Monthly mean sea level at Andreia, Russian Federation.

provided by the Permanent Service for Mean Sea Level (PSMSL) ${ }^{5}$ (Holgate et al., 2013).

For example, Fig. 26a shows the monthly MSL in $\mathrm{mm}$ from the tide gauge at Andreia in the Russian Federation. While it is easily noticed that the time series differs from a GPS position time series, this difference is less evident when comparing it to the gravity time series in Fig. 24. This comparison suggest the presence of timecorrelated noise also in the MSL record which is confirmed when looking at the power spectrum in Fig. 26b, showing a power-law spectrum. However, at the time of this study the PSMSL did not provide a full stochastic analysis of the MSL records and only considered white noise when estimating the parameters and associated uncertainties. As this spectrum is not an isolated case, rather it is representative of many stations in the PSMSL database, the PSMSL has updated its analysis strategy recently, see https://www.psmsl.org/products/trends/methods.php. Nevertheless, for demonstration purposes only we will use here the trend estimates from the PSMSL assuming randomness.

Fig. 27 shows the distribution of all tide gauges contributing to the PSMSL as in 2014. The differences in coverage between the Northern and Southern Hemispheres

${ }^{5}$ Data available at http://www.psmsl.org/ 


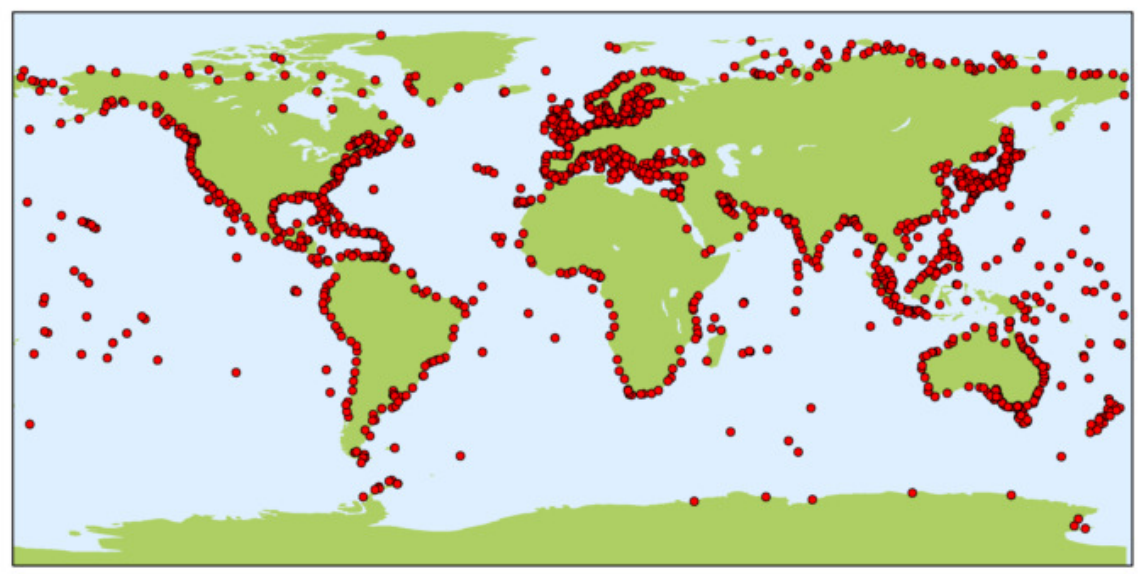

Fig. 27: Distribution of PSMSL tide gauge stations as in 2014. (Reproduced from https://www.psmsl.org.)

are clearly evident. Furthermore, it is known that most of the long MSL records (40+ years) are located around Northern Europe and the Baltic Sea with a few stations in North America, Asia and Australia.

In order to construct time series of sea level measurements at each station, the monthly means have to be reduced to a common datum. This reduction is performed by the PSMSL making use of the tide gauge datum history provided by the supplying authority. To date, approximately two thirds of the stations in the PSMSL database have had their data adjusted in this way, forming the RLR dataset. Only the RLR data set was used in this analysis as suggested by the PSMSL.

The histogram of the time series lengths in Fig. 28 shows they span from a few months to around 200 years, although the mode is suggested to be centred around 20 years.

Besides the annual and semi-annual periodic terms observed in GPS time series, the MSL records are also influenced by other time-scale phenomena, some of them spanning several years like the Rossby wave propagation from open ocean towards the shore (Douglas et al., 2001; Holgate and Woodworth, 2004), the El Niño phenomenon in the Pacific Ocean (White et al., 2005; Church and White, 2006) or the 18.6 year Lunar Nodal Cycle (Baart et al., 2012).

There are few sea level studies which have considered time-correlation within the noise of the MSL records (Harrison, 2002; Mazzotti et al., 2008; Hughes and Williams, 2010; Burgette et al., 2013), hence the interest of the MCMC analysis on these time series.

Besides a purely scientific goal, sea-level rise is of importance nowadays due to the socio-economic impact it will have on millions of people who live in coastal 


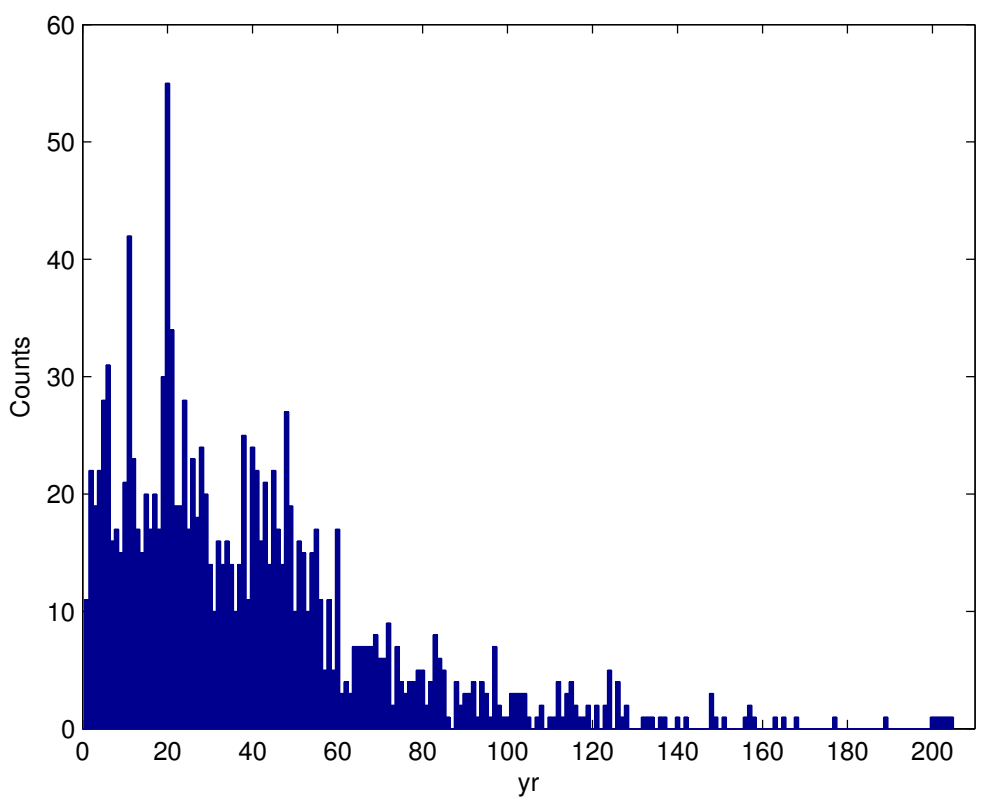

Fig. 28: Histograms for all the monthly RLR MSL records from PSMSL.

regions around the world. Projections of sea level for the $21^{s t}$ century help to prepare governments and people in these regions. The projections have uncertainties which depend to a degree on the noise within the time series, therefore, it is necessary to understand the nature of this noise.

Like other geophysical time series, MSL records have a power-law spectrum (Agnew, 1992; Harrison, 2002; Mazzotti et al., 2008). Nevertheless, so far it has not been common to use models of white noise plus power-law in the analyses of MSL records, with a few exceptions (Mazzotti et al., 2008; Hughes and Williams, 2010; Burgette et al., 2013). In order to estimate the spectral index and its effect on the estimated parameters and their uncertainties, an analysis using the MCMC method implemented has been carried out on the MSL records provided by the PSMSL. As in Hughes and Williams (2010), the chosen deterministic model includes linear plus annual and semi-annual terms.

As Zhang et al. (1997); Mao et al. (1999) and Williams et al. (2004) showed for GPS position time series, the hypothesis of a pure white noise process clearly underestimates the uncertainties of the parameters. Moreover, unlike white noise, long memory processes have a power-law spectrum and, consequently, as the amplitude changes in time, even the estimated parameters themselves may be different (Harrison, 2002). 


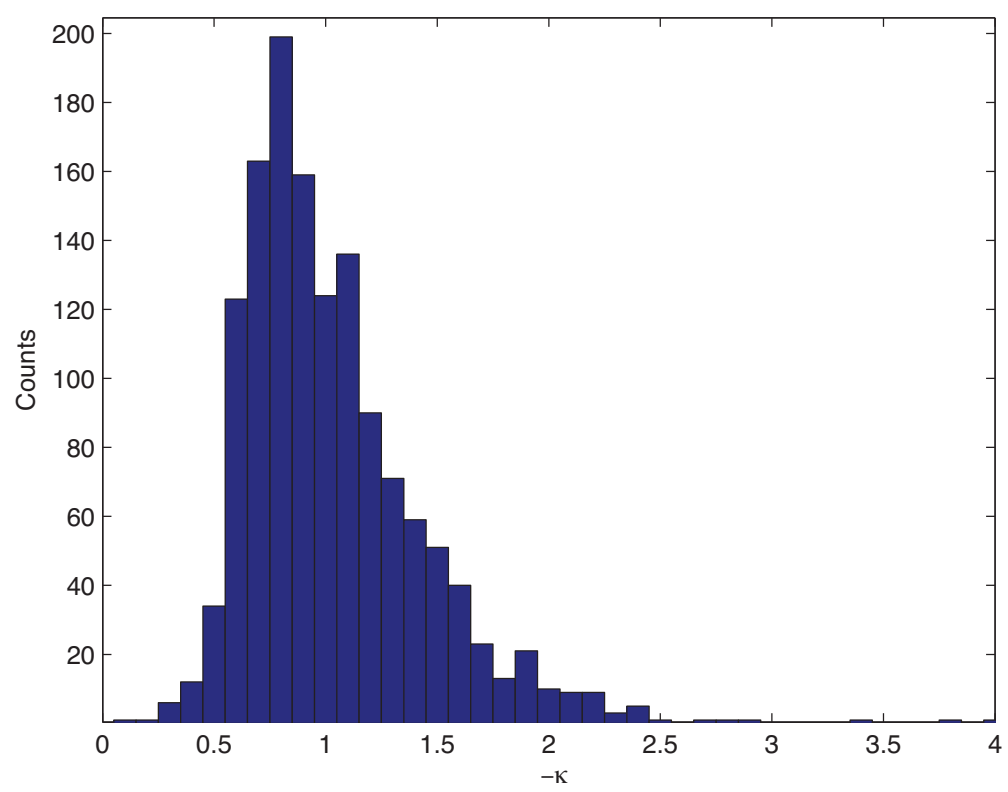

Fig. 29: Estimates for $-\kappa$ for monthly MSL records from the PSMSL RLR data base.

Therefore, differences coming from this analysis on the MSL time series are expected, and they may affect conclusions of other applications where their trends are used, as, for example, the computation of vertical land movements from GPS time series for the correction of tide gauge records in sea level studies, e.g. Wöppelmann et al. (2007).

Fig. 29 shows the distribution of the spectral index $-\kappa$ for the MSL data set. Clearly, most of their values do contain coloured noise. They are centred near $-\kappa=1$ (i.e. Flicker noise) and, in general, the value of $-\kappa$ ranges from $\sim 0$ to above 2 , spanning the stationary and non-stationarity regimes. This is in good agreement with Burgette et al. (2013) where they carried out an analysis on MSL records from Australia with CATS, and found that most of the MSL spectrums fit either a combination of white noise and power-law, or a First Order Gauss Markov process (which is equivalent to a Random-Walk at middle frequency).

The empirical cumulative density function (ECDF) of $-\kappa$ is shown in Fig. 30. Most remarkable is that $99 \%$ of the MSL records have an $-\kappa>0.5$, with $56 \%$ in the interval $[0.5-1)$ (stationary regimes) and $44 \%$ in the non-stationary regimes, i.e. with $-\kappa \geq 1$. 


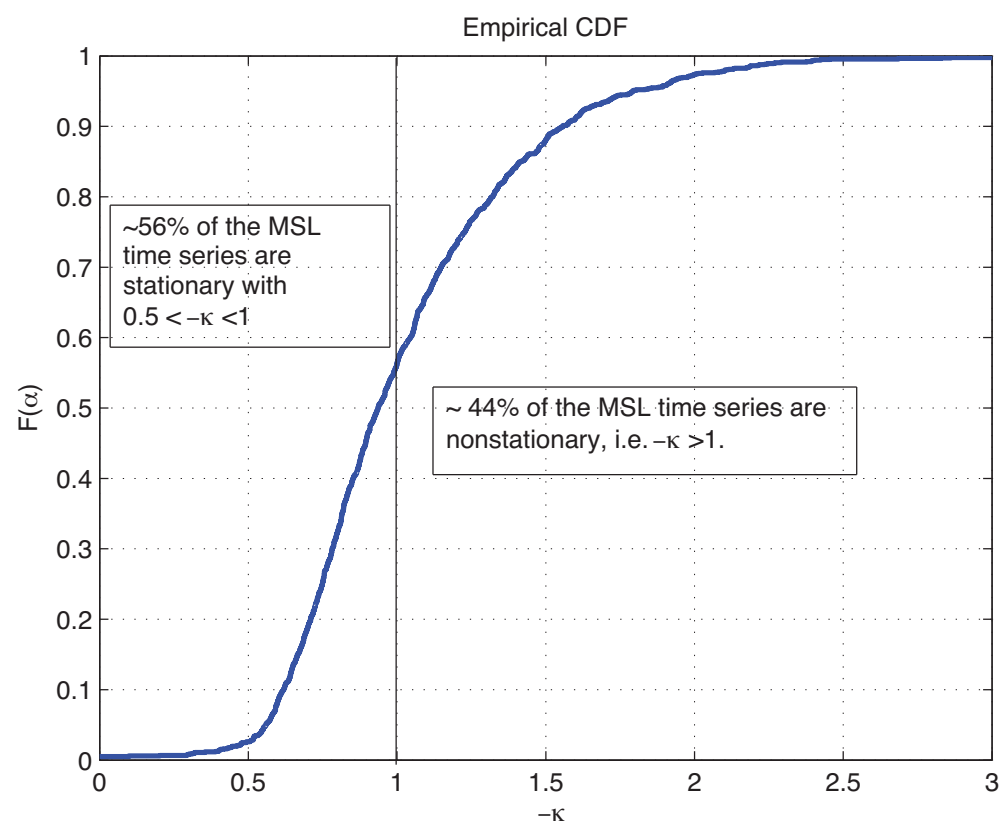

Fig. 30: Empirical cumulative density function of $-\kappa$.

In order to estimate the trends, only monthly MSL records longer than 30 years were analysed, assuming most of the seasonal effects are sub-monthly (therefore they are filtered out), semi-annual and annual, though there are still some discrepancies about oscillations of longer periods within the time series (Chambers et al., 2012). According to Woodworth et al. $(1999,2009)$ and cites therein, this is the length required to estimate sea level trends with a standard error of the order of $0.5 \mathrm{~mm} / \mathrm{yr}$ or less.

A histogram of the estimated velocity $v$ is shown in Fig. 31. Although most of the estimated values are centered around $v=1 \mathrm{~mm} / \mathrm{yr}$, they range from $-10 \mathrm{~mm} / \mathrm{yr}$ to $10 \mathrm{~mm} / \mathrm{yr}$, with some extreme cases at $-20 \mathrm{~mm} / \mathrm{yr}$ and $20 \mathrm{~mm} / \mathrm{yr}$. The median is $v_{\text {med }}=1.26 \mathrm{~mm} / \mathrm{yr}$ and $v_{\text {med }}=1.41 \mathrm{~mm} / \mathrm{yr}$ for the MCMC and PSMSL cases, respectively. Their differences in their standard deviation are also sub-millimetre, namely $\sigma_{v}=3.21 \mathrm{~mm} / y r$ and $\sigma_{v}=2.83 \mathrm{~mm} / \mathrm{yr}$ for MCMC and PSMSL, respectively. As these values are corresponding to sea level variations potentially affected by vertical land movements, they cannot be compared with a globally averaged sea level rise estimate. 


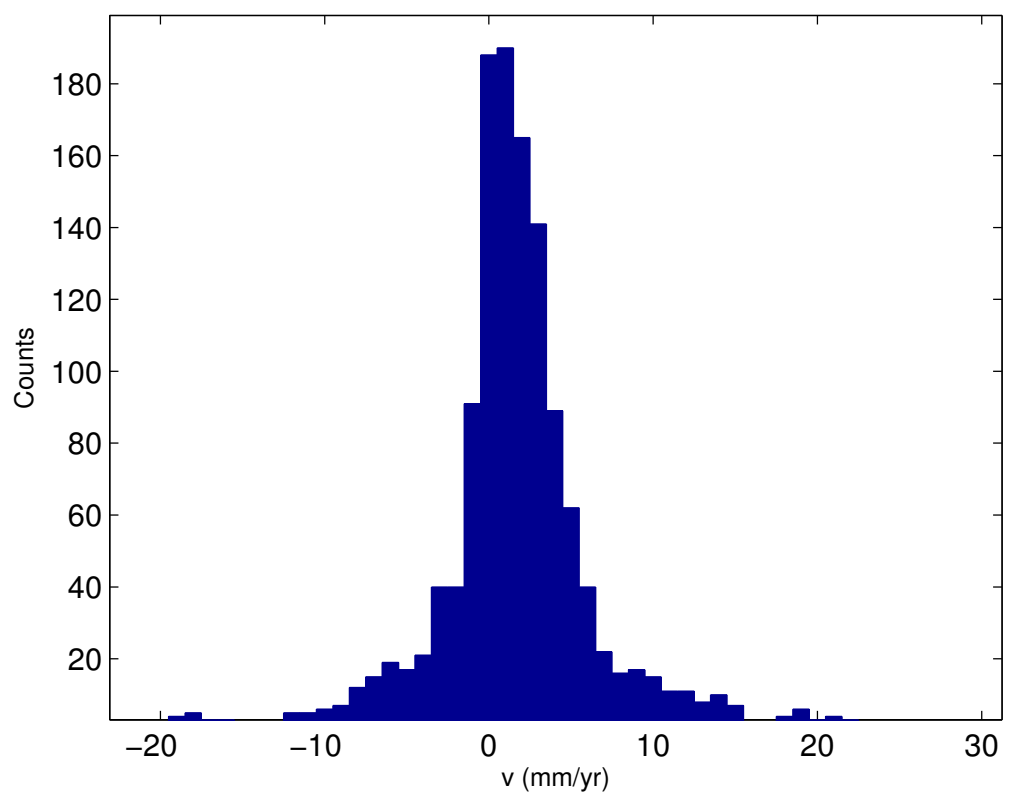

Fig. 31: Estimates of $v$ for monthly MSL records from the PSMSL RLR data base.

Finally, the uncertainties of the estimated $v$ from the MCMC method are shown in Fig. 32. Although the estimates range from 0 to $35 \mathrm{~mm} / \mathrm{yr}$, they cluster at the few millimetre level, mainly between 0 and $5 \mathrm{~mm} / \mathrm{yr}$.

In order to compare the results from the MCMC analysis with the values from PSMSL (white noise model), the same stations for which the trends are given in the PSMSL web page ${ }^{6}$ were selected. Fig. 33 shows the differences. In the vertical axis, the estimates from MCMC (coloured noise hypothesis); in the horizontal axis, white noise model is assumed. Although most of them align along the diagonal, there are some noticeable differences with some points around $(5,17) \mathrm{mm} / \mathrm{yr}$ and $(5,-20) \mathrm{mm} / \mathrm{yr}$. It is worth mentioning that most of the distant points from the diagonal have $-\kappa \geq 1$ (red-circled points in Fig. 33), i.e. indicating non-stationary processes. This is in good agreement with the fact that such processes, i.e. those for which $-\kappa \geq 1$, contribute to the velocity. This could be the reason for those extreme values below the diagonal, as the velocity from the long-memory process could be negative. Moreover, as it was explained in Harrison (2002), due to the power-law spectrum, as the perturbations at low-frequency are included with longer time series, they naturally contribute to increase the estimated velocity.

${ }^{6}$ Data available at http://www.psmsl.org/ 


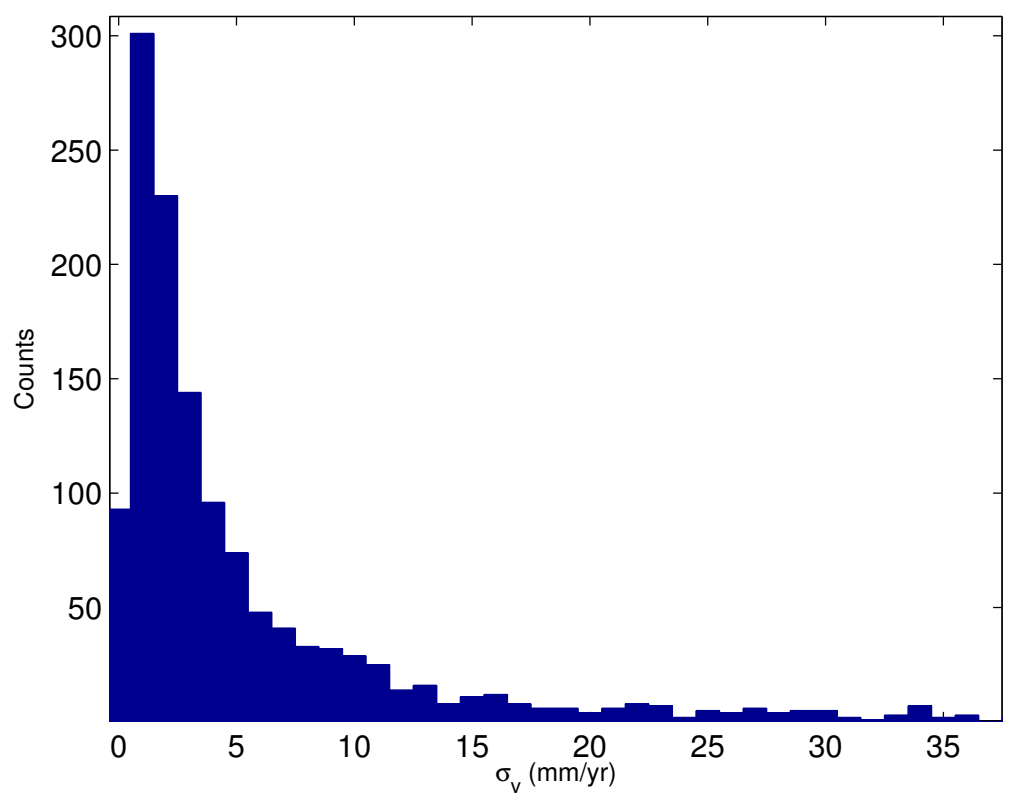

Fig. 32: Estimates of $\sigma_{v}$ for monthly MSL records from the PSMSL RLR data base.

The uncertainties from both methods are compared in Fig. 34. In general, as expected with a few exceptions, the estimates from MCMC are larger than those from the PSMSL model, i.e. they are well above the diagonal. A plot of the ECDF of the ratio of both uncertainties $R_{\sigma_{v}} \equiv \sigma_{v}(M C M C) / \sigma_{v}(P S M S L)$ in Fig. 35 provides more information. Around $87 \%$ of the uncertainties estimated by the MCMC method are larger than those from the PSMSL model. Moreover, $86 \%$ of the uncertainties estimated with the MCMC method are [1 - 10] times larger than those obtained with a pure white-noise model.

According to these results, to consider a coloured noise instead of white noise model, and analyse the monthly MSL records with the MCMC model, yields some quantitative differences, namely:

- Due to the contribution of the non-stationary noise to the trend of some MSL records larger absolute velocities are estimated (see Fig. 33).

- $86 \%$ of the stations have uncertainties which are [1 - 10] times larger than those from the white noise model. 


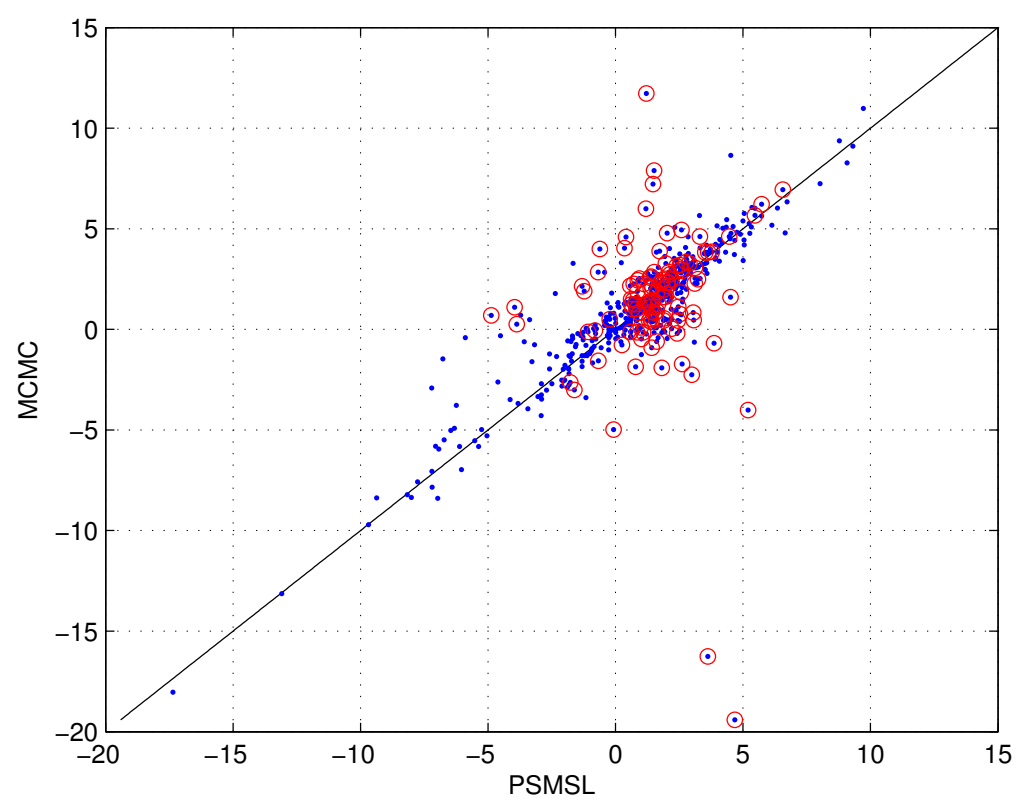

Fig. 33: Estimates for $v(\mathrm{~mm} / \mathrm{yr})$ from MCMC vs PSMSL models. Red-circled points stem from MSL records with $-\kappa \geq 1$.

The uncertainties here just consider the effect from the coloured noise. Corrections with GPS velocities would increase the uncertainty of any "averaged" sea level change estimate (Wöppelmann et al., 2007, 2009; Santamaría-Gómez et al., 2012).

The MCMC analysis carried out on the monthly MSL records available in the PSMSL RLR data base confirms that the assumption of white noise, as it was considered in the past, does not hold for these either. Most of the time series have $-\kappa \sim 1$ (see Fig. 29), and around 3\% of them have $-\kappa \geq 2$ (see Fig 30). Moreover, $44 \%$ of them are non-stationary as their spectral indices are $-\kappa \geq 1$. Consequently, different velocities were found with the coloured noise model (see red-circled points in Fig. 33).

Once again, the uncertainties from the MCMC method are larger as Fig. 34 shows. Actually, around $86 \%$ of the MSL records analysed with MCMC have uncertainties [1 - 10] times larger than for the white noise-only model.

Moreover, the analysis of MSL records has shown that a pure white-noise model underestimates the uncertainties ([1 - 10] times smaller). Unlike for the analysis of GPS time series, the range of the ratio for the uncertainties is very wide, hence the lack of any transformation method. Therefore, the authors suggest to analyse all MSL records with the MCMC method. 


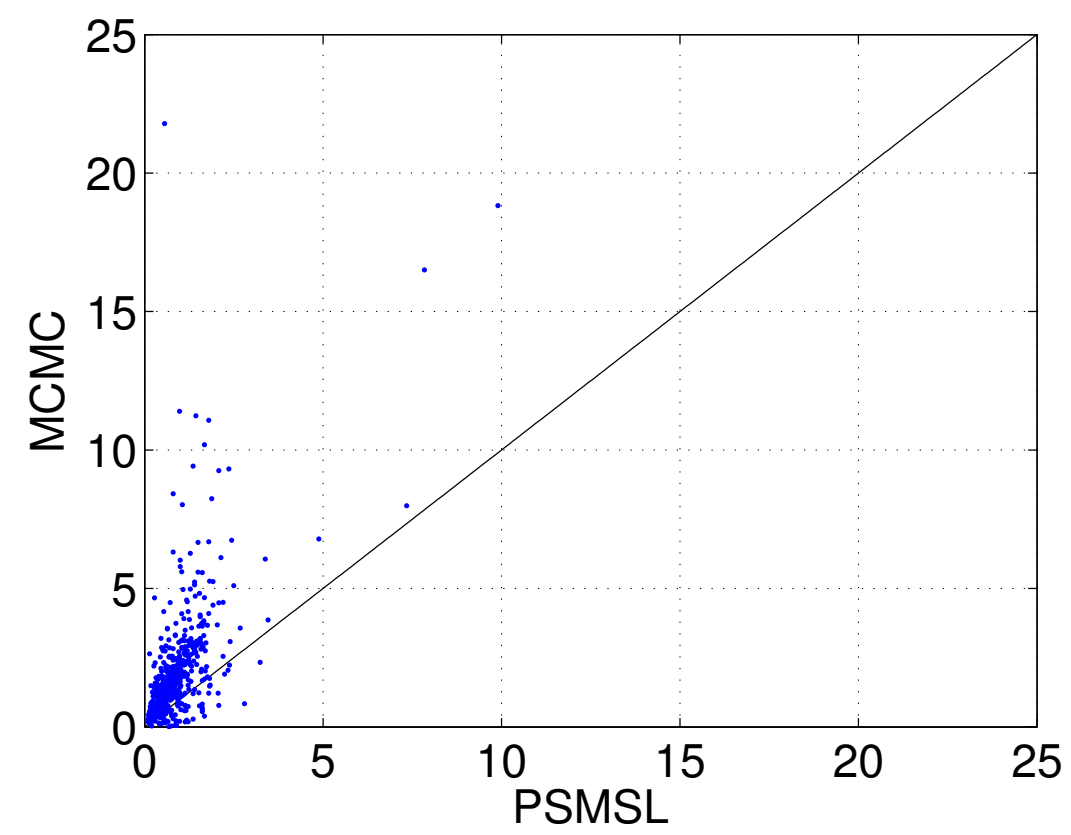

Fig. 34: Estimates for $\sigma_{v}(\mathrm{~mm} / \mathrm{yr})$ from MCMC vs PSMSL models.

The different trends estimated for some MSL records are the consequence of the contribution of the non-stationary power-law noise, i.e. those for which $-\kappa \geq 1$. Indeed, if the model used to fit the records does not contain any velocity term that comes from the noise it will yield an underestimated spectral index.

The analysis with the MCMC method carried out on the MSL records from the PSMSL RLR data set shows that considering coloured noise instead of a pure white noise model yields some quantitative differences, namely:

- Larger absolute velocities due to the contribution of the non-stationary processes to the trend (see Fig. 33).

- Larger uncertainties which are $[1-10]$ times larger than those from the white noise model for $86 \%$ of the analysed MSL records. This is a similar range as was stated for GPS time series in Mao et al. (1999).

From these results it is advisable to take into account the temporally-correlated noise within the MSL records, otherwise, it would yield biased estimated trends and underestimated uncertainties for sea level studies. 


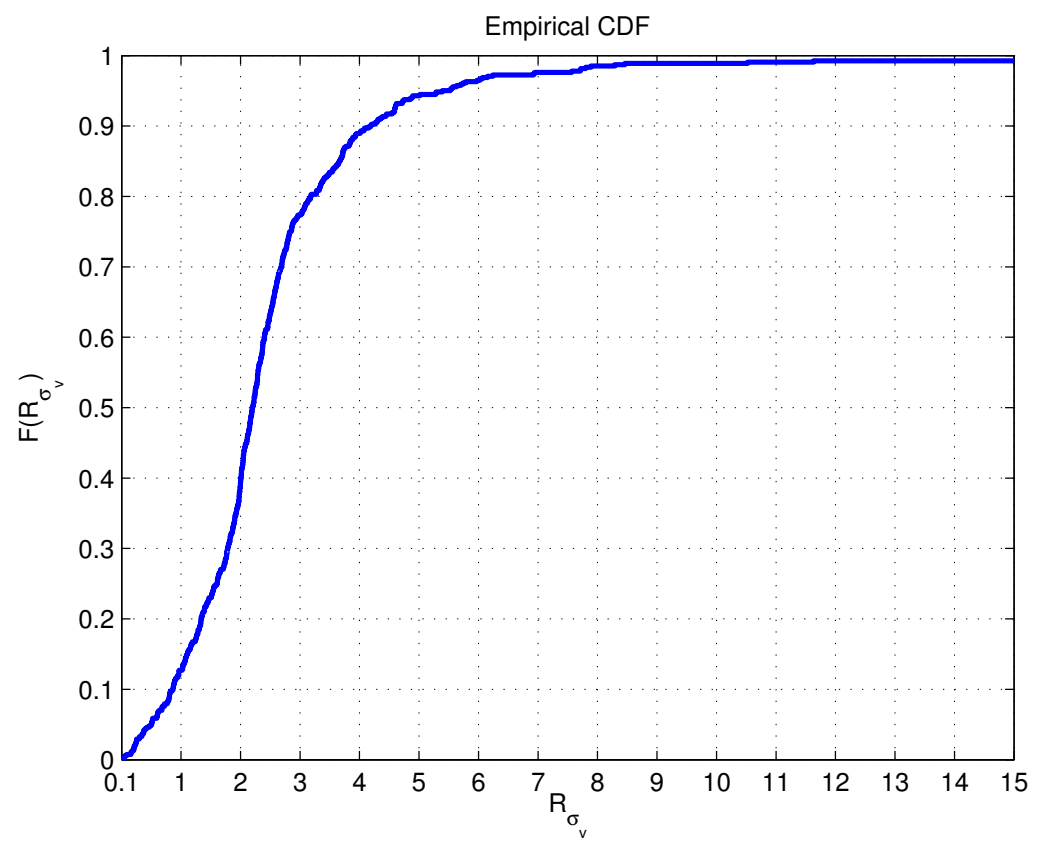

Fig. 35: Empirical CDF of $\sigma_{v}^{M C M C} / \sigma_{v}^{P S M S L}$. 


\section{Summary}

In order to better constrain geophysical models using time series of geodetic observations, e.g., GPS-derived positions, superconducting gravity and mean sea level, it is necessary to have an estimate of the stochastic properties of the series. Due to the fractal nature of different geophysical phenomena (Agnew, 1992; Mandelbrot, 1982), both the deterministic and stochastic models have to be estimated and the a priori assumption of white noise is no longer valid. Doing so would underestimate the uncertainties of the parameter estimates (Zhang et al., 1997; Mao et al., 1999; Williams, 2003a; Williams and Willis, 2006). Moreover, even the trend of the time series, which provides insight about geophysical variable rates, could be affected should the noise be non-stationary (Harrison, 2002).

In this regard methods, such as the presented Markov Chain Monte Carlo (MCMC) method, that provide a sample of the distribution function of the parameters and the noise are valid for geodetic time series of stochastic phenomena.

In this chapter several examples as to how to implement statistical analysis of geodetic time series by means of the MCMC method have been presented, namely, GPS position time series (with synthetic and real data sets), superconducting gravity time series and mean sea level records. Furthermore, the impact of the MCMCderived GPS station velocities and uncertainties on constraints of plate motion models was demonstrated.

The results from synthetic data prove that the MCMC method performs well. In general, the true values are within the $1 \sigma$ confidence level. It can also be stated from those results that the wider the parameter space, i.e. the more parameters, the larger the uncertainties.

The MCMC method provides samples of the distributions of the estimates, thus through histograms it is easy to obtain statistical information about them as, for example, the mean, the median, the uncertainties at different confidence levels, and the cross-correlation between them.

It has also been noted that the MCMC method seems to be a good estimator for non-stationary time series, though without giving a mathematical proof for this in this study.

According to Fig. 7, CATS performs alike in the non-stationary regime, though at low values provides biased $-\kappa$ estimates as a consequence of setting $\sigma_{w n}$ to zero when it is difficult for the estimator to distinguish one noise source from the other.

Another advantage of MCMC upon CATS is that the former does not deal with derivatives of the covariance matrix as, for example, the Fisher matrix, thus avoiding numerical issues present in some results from CATS (e.g. a $\mathrm{NaN}$ for the uncertainty of the East component velocity for THU3).

The analysis carried out on the synthetic data leads to the following conclusions:

- Overall, the model parameter estimates from both methods are in good agreement.

- The MCMC method estimates similar $\sigma_{p l}$ and larger $-\kappa, \sigma_{w n}$ with smaller RMS than CATS. 
- The MCMC method provides larger uncertainties for the model parameters, e.g. $\sigma_{v}(M C M C) \sim 1.40 \times \sigma_{v}(C A T S)$.

- Though the correlation parameters $a$ and $b$ are useful to transform estimates from one method into the other, in the order to compare both methods, it is better (more robust) to compute the median than the mean, since the latter is more easily corrupted by the presence of outliers.

The larger estimates for the spectral index $-\kappa$ from MCMC than from CATS indicate that, according to the MCMC method, there is more time-correlation within the noise than what CATS suggests. On the other hand, the values of $\sigma_{p l}$ obtained from CATS are overestimated, for, even though they are larger than those from MCMC, the RMS for $\sigma_{p l}$ from CATS is $\sim 20 \%$ larger than that for the MCMC estimate.

Finally, the RMS of the estimates of the white noise amplitude $\sigma_{w n}$ from the MCMC method is around half the value of its counterpart from CATS. CATS sets $\sigma_{w n}$ to zero for low values of $-\kappa$ (red-circled points in various figures), thus underestimating the temporal-correlation within the time series and, consequently, underestimating the uncertainties of the model parameters as well. Despite of the larger values for $\sigma_{p l}$ from CATS than from MCMC, as $\sigma_{v}$ increases geometrically with larger $-\kappa$ and linearly with the power amplitude, the larger $-\kappa$ estimates from MCMC lead to larger uncertainties for the velocity estimate.

Concerning the uncertainties for the other model parameters, $y_{0}$ is smaller $R_{y_{0}}=$ 0.70 for MCMC than for CATS, and the periodic terms have uncertainties that range between 1.03 and 1.08 times larger for the MCMC than for the CATS method. Except for $\sigma_{v}$ and $\sigma_{y_{0}}$ with $\Delta_{v}=0.23 \mathrm{~mm} / \mathrm{yr}$ and $\Delta_{y_{0}}=-0.88 \mathrm{~mm}$, the differences of the periodic terms are sub-millimetre.

Results obtained from the JPL data set are in good agreement with those for the synthetic data. As the estimated $-\kappa$ are larger for the MCMC method than for CATS, the former method yields larger uncertainties for the parameters of the model. Namely, for the uncertainties of the estimated velocity of the JPL data sets

- $\sigma_{v}(M C M C) \sim[1.18-1.40] \times \sigma_{v}(C A T S)$, which is in good agreement with the results for the synthetic data set.

Similar results are obtained for the estimates of the amplitudes of the periodic terms in the JPL time series. All the uncertainties are larger for the MCMC than for the CATS method. In general, the uncertainties are $1.03-1.11$ times larger for MCMC than for CATS. This is in consistent with the results from the synthetic data set too.

Finally, as the estimates of both methods cluster along a straight line, a method to transform estimated parameters and uncertainties from CATS to MCMC has been introduced. Nevertheless, it is a more robust method to use the median of the ratio and of the differences instead of the mean.

Constraints of Plate Motion Models is another example of applications of MCMC methods for geodetic time series. Both methods, i.e. MCMC and CATS, yield results with differences at $1 \sigma$ confidence level. Such differences may imply different values for the constraints in geophysical models. Moreover, according to the reduced $\chi^{2}$, the MCMC method yields less underestimated uncertainties than CATS, as the 
uncertainties of the former should be 22.4 times larger, whereas for the latter they should be 28.3 larger, in order to get the best fit possible, i.e. $\chi^{2}=1$.

The MCMC analysis of the time series of the superconducting gravity measurements has shown another advantage of using an integrator method such as MCMC. Due to the characteristics of the algorithm, it explores the surrounding areas of a maximum, thereby spotting other local maxima. This is the case for the ordinate parameter $y_{0}$ of the gravity time series. This is typical of non-stationary stochastic processes, where the noise adds some velocity into the trend. Therefore, it us suggested to use the MCMC method in time series with high spectral index, e.g. $-\kappa>1$.

Finally, the analysis of tide gauge records has confirmed other findings that in order to take into account the temporally-correlated noise within the series, it is necessary to estimate the spectral index and its uncertainty, otherwise it may yield biased trend estimates and underestimated uncertainties for sea level studies. 


\section{Appendices}

The appendices first present the station-specific information used in the plate motion model estimation with the horizontal station velocities from both the MCMC and CATS methods as described in Section 4.2. Then in a second appendix a crossevaluation of the MCMC and Hector methods using the Benchmark Synthetic GNSS (BSG) time series (Chapter 2 of this book) is detailed and discussed.

\section{A Station Information for Plate Motion Model Estimation}


Table 15: Station Information and Velocity Estimates from the MCMC method.

\begin{tabular}{|c|c|c|c|c|c|c|c|c|c|}
\hline \multirow[b]{2}{*}{ Plate } & \multicolumn{3}{|c|}{ Station } & \multicolumn{4}{|c|}{ Horizontal Velocities (mm/yr) } & \multicolumn{2}{|c|}{ Residuals (mm/yr) } \\
\hline & ID & $\lambda(\mathrm{deg})$ & $\phi(\mathrm{deg})$ & $V_{N}$ & $V_{E}$ & $\sigma_{V_{N}}$ & $\sigma_{V_{E}}$ & $\mathrm{~N}$ & $\mathrm{E}$ \\
\hline Amurian & CHAN & 125.44 & 43.79 & -12.71 & 26.24 & 1.22 & 0.33 & 1.81 & 0.97 \\
\hline Amurian & KHAJ & 135.05 & 48.52 & -13.63 & 21.65 & 0.29 & 0.14 & 1.08 & 0.02 \\
\hline Antartica & SYOG & 39.58 & -69.01 & 2.89 & -4.04 & 0.07 & 0.08 & -0.03 & 0.04 \\
\hline Antartica & DAV1 & 77.97 & -68.58 & -5.29 & -3.10 & 0.07 & 0.21 & -0.19 & 0.11 \\
\hline Antartica & CAS1 & 110.52 & -66.28 & -10.03 & 1.86 & 0.14 & 0.13 & 0.26 & 0.07 \\
\hline Antartica & DUM1 & 140.00 & -66.67 & -11.42 & 9.54 & 0.39 & 1.06 & 0.82 & 2.45 \\
\hline Antartica & VESL & -2.84 & -71.67 & 10.28 & -0.30 & 0.09 & 0.06 & 0.09 & 0.01 \\
\hline Arabia & HALY & 36.10 & 29.14 & 22.59 & 26.73 & 0.79 & 0.57 & -1.77 & 1.07 \\
\hline Arabia & BHR2 & 50.61 & 26.21 & 30.04 & 31.39 & 0.14 & 0.21 & 0.39 & 0.62 \\
\hline Arabia & YIBL & 56.11 & 22.19 & 31.57 & 32.97 & 0.21 & 0.30 & 0.39 & -1.24 \\
\hline Australia & YAR1 & 115.35 & -29.05 & 57.30 & 39.02 & 0.31 & 0.50 & -1.19 & -0.83 \\
\hline Australia & NNOR & 116.19 & -31.05 & 57.94 & 38.41 & 0.54 & 0.16 & -0.73 & -0.72 \\
\hline Australia & KARR & 117.10 & -20.98 & 58.36 & 38.93 & 0.20 & 0.12 & -0.53 & -0.89 \\
\hline Australia & DARW & 131.13 & -12.84 & 59.28 & 36.23 & 0.14 & 0.20 & -0.60 & -0.40 \\
\hline Australia & CEDU & 133.81 & -31.87 & 58.81 & 29.08 & 0.18 & 0.20 & -0.81 & -0.28 \\
\hline Australia & ALIC & 133.89 & -23.67 & 59.67 & 32.11 & 0.32 & 0.11 & 0.03 & -0.54 \\
\hline Australia & ADE1 & 138.65 & -34.73 & 58.35 & 24.98 & 0.18 & 0.21 & -0.53 & -0.24 \\
\hline Australia & TOW2 & 147.06 & -19.27 & 55.77 & 28.86 & 0.14 & 0.11 & -0.90 & -0.89 \\
\hline Australia & НOB2 & 147.44 & -42.80 & 55.70 & 14.40 & 0.34 & 0.05 & -0.77 & -0.24 \\
\hline Australia & PARK & 148.26 & -33.00 & 52.97 & 18.92 & 0.41 & 0.45 & -3.24 & -1.98 \\
\hline Australia & TIDB & 148.98 & -35.40 & 55.26 & 18.26 & 0.13 & 0.11 & -0.68 & -0.62 \\
\hline Australia & STR1 & 149.01 & -35.32 & 55.31 & 18.65 & 0.18 & 0.10 & -0.62 & -0.27 \\
\hline Australia & SYDN & 151.15 & -33.78 & 54.27 & 18.06 & 0.42 & 0.22 & -0.82 & -0.76 \\
\hline Australia & SUNM & 153.04 & -27.48 & 54.06 & 21.91 & 0.34 & 0.50 & -0.23 & -0.44 \\
\hline Australia & KOUC & 164.29 & -20.56 & 47.73 & 22.75 & 0.21 & 0.30 & -0.58 & -0.74 \\
\hline Australia & NOUM & 166.41 & -22.27 & 45.77 & 20.57 & 0.29 & 0.18 & -1.19 & -0.85 \\
\hline Australia & AUCK & 174.83 & -36.60 & 39.74 & 4.52 & 0.11 & 0.14 & -1.23 & -0.32 \\
\hline
\end{tabular}


Table 15 - continued from previous page

\begin{tabular}{|c|c|c|c|c|c|c|c|c|c|}
\hline \multirow[b]{2}{*}{ Plate } & \multicolumn{3}{|c|}{ Station } & \multicolumn{4}{|c|}{ Horizontal Velocities $(\mathrm{mm} / \mathrm{yr})$} & \multicolumn{2}{|c|}{ Residuals (mm/yr) } \\
\hline & ID & $\lambda(\mathrm{deg})$ & $\phi(\mathrm{deg})$ & $V_{N}$ & $V_{E}$ & $\sigma_{V_{N}}$ & $\sigma_{V_{E}}$ & $\mathrm{~N}$ & $\mathrm{E}$ \\
\hline Caribbean & CRO1 & -64.58 & 17.76 & 13.57 & 10.79 & 0.10 & 0.33 & 0.04 & -1.69 \\
\hline Eurasia & HERS & 0.34 & 50.87 & 16.41 & 16.50 & 0.17 & 0.17 & -0.04 & -0.36 \\
\hline Eurasia & EBRE & 0.49 & 40.82 & 15.81 & 19.84 & 0.14 & 0.08 & -0.64 & 0.66 \\
\hline Eurasia & SHEE & 0.74 & 51.45 & 16.65 & 16.71 & 0.29 & 0.09 & 0.23 & -0.09 \\
\hline Eurasia & BELL & 1.40 & 41.60 & 15.79 & 19.56 & 0.29 & 0.10 & -0.60 & 0.35 \\
\hline Eurasia & TOUL & 1.48 & 43.56 & 16.85 & 20.01 & 0.49 & 0.44 & 0.47 & 1.19 \\
\hline Eurasia & OPMT & 2.33 & 48.84 & 15.72 & 18.15 & 0.13 & 0.10 & -0.59 & 0.35 \\
\hline Eurasia & MALL & 2.62 & 39.55 & 16.21 & 19.82 & 0.19 & 0.23 & -0.09 & 0.00 \\
\hline Eurasia & SJDV & 4.68 & 45.88 & 16.06 & 19.47 & 0.09 & 0.09 & -0.06 & 0.52 \\
\hline Eurasia & REDU & 5.14 & 50.00 & 15.57 & 18.29 & 0.12 & 0.14 & -0.51 & 0.17 \\
\hline Eurasia & MARS & 5.35 & 43.28 & 15.96 & 20.07 & 0.08 & 0.09 & -0.11 & 0.45 \\
\hline Eurasia & KOSG & 5.81 & 52.18 & 16.04 & 17.96 & 0.08 & -0.00 & 0.02 & 0.22 \\
\hline Eurasia & WSRT & 6.60 & 52.91 & 16.41 & 17.64 & 0.10 & 0.06 & 0.48 & -0.09 \\
\hline Eurasia & BORK & 6.75 & 53.56 & 15.22 & 17.58 & 0.25 & 0.10 & -0.71 & -0.03 \\
\hline Eurasia & WAB2 & 7.46 & 46.92 & 15.84 & 19.87 & 0.12 & 0.13 & -0.02 & 0.57 \\
\hline Eurasia & ZIMM & 7.47 & 46.88 & 16.23 & 19.59 & 0.10 & 0.05 & 0.37 & 0.29 \\
\hline Eurasia & IENG & 7.64 & 45.02 & 15.44 & 20.47 & 0.15 & 0.13 & -0.40 & 0.75 \\
\hline Eurasia & HELG & 7.89 & 54.17 & 15.89 & 17.57 & 0.09 & 0.14 & 0.08 & -0.14 \\
\hline Eurasia & AJAC & 8.76 & 41.93 & 16.42 & 21.44 & 0.10 & 0.30 & 0.69 & 0.93 \\
\hline Eurasia & РTBB & 10.46 & 52.30 & 15.55 & 18.81 & 0.11 & 0.08 & 0.02 & 0.08 \\
\hline Eurasia & WARN & 12.10 & 54.17 & 15.58 & 18.32 & 0.11 & 0.08 & 0.25 & -0.32 \\
\hline Eurasia & BUDP & 12.50 & 55.74 & 14.92 & 18.05 & 0.16 & 0.07 & -0.36 & -0.30 \\
\hline Eurasia & WTZR & 12.88 & 49.14 & 15.56 & 20.39 & 0.07 & 0.08 & 0.32 & 0.46 \\
\hline Eurasia & POTS & 13.07 & 52.38 & 15.57 & 19.16 & 0.23 & 0.11 & 0.36 & -0.09 \\
\hline Eurasia & SASS & 13.64 & 54.51 & 14.65 & 19.02 & 0.20 & 0.08 & -0.48 & 0.13 \\
\hline Eurasia & GOPE & 14.79 & 49.91 & 15.10 & 19.99 & 0.15 & 0.12 & 0.11 & -0.16 \\
\hline Eurasia & GRAZ & 15.49 & 47.07 & 15.38 & 21.74 & 0.11 & 0.00 & 0.49 & 0.90 \\
\hline Eurasia & WROC & 17.06 & 51.11 & 14.69 & 20.18 & 0.10 & 0.07 & 0.02 & -0.17 \\
\hline Eurasia & BOR1 & 17.07 & 52.28 & 14.58 & 20.01 & 0.06 & 0.08 & -0.08 & -0.10 \\
\hline
\end{tabular}


Table 15 - continued from previous page

\begin{tabular}{|c|c|c|c|c|c|c|c|c|c|}
\hline \multirow[b]{2}{*}{ Plate } & \multicolumn{3}{|c|}{ Station } & \multicolumn{4}{|c|}{ Horizontal Velocities (mm/yr) } & \multicolumn{2}{|c|}{ Residuals (mm/yr) } \\
\hline & ID & $\lambda(\operatorname{deg})$ & $\phi(\mathrm{deg})$ & $V_{N}$ & $V_{E}$ & $\sigma_{V_{N}}$ & $\sigma_{V_{E}}$ & $\mathrm{~N}$ & $\mathrm{E}$ \\
\hline Eurasia & PENC & 19.28 & 47.79 & 14.67 & 22.15 & 0.16 & 0.07 & 0.34 & 0.73 \\
\hline Eurasia & LAMA & 20.67 & 53.89 & 14.26 & 20.10 & 0.56 & 0.08 & 0.16 & -0.38 \\
\hline Eurasia & JOZE & 21.03 & 52.10 & 14.39 & 21.03 & 0.10 & 0.07 & 0.34 & 0.10 \\
\hline Eurasia & BOGO & 21.04 & 52.48 & 14.42 & 20.47 & 0.15 & 0.02 & 0.38 & -0.38 \\
\hline Eurasia & KLPD & 21.12 & 55.72 & 13.36 & 20.11 & 0.48 & 0.53 & -0.67 & -0.06 \\
\hline Eurasia & UZHL & 22.30 & 48.63 & 13.90 & 21.84 & 0.10 & 0.13 & 0.06 & 0.02 \\
\hline Eurasia & SULP & 24.01 & 49.84 & 13.96 & 21.45 & 0.13 & 0.14 & 0.41 & -0.47 \\
\hline Eurasia & RIGA & 24.06 & 56.95 & 13.44 & 20.17 & 0.09 & 0.00 & -0.09 & -0.31 \\
\hline Eurasia & GLSV & 30.50 & 50.36 & 12.83 & 22.38 & 0.11 & 0.12 & 0.51 & -0.57 \\
\hline Eurasia & MIKL & 31.97 & 46.97 & 12.03 & 23.53 & 0.19 & 0.15 & 0.01 & -0.17 \\
\hline Eurasia & CRAO & 33.99 & 44.41 & 11.43 & 24.00 & 0.22 & 0.67 & -0.17 & -0.32 \\
\hline Eurasia & KHAR & 36.24 & 50.01 & 11.93 & 24.25 & 0.23 & 0.25 & 0.83 & 0.35 \\
\hline Eurasia & MOBN & 36.57 & 55.11 & 11.83 & 22.77 & 0.16 & 0.24 & 0.81 & -0.32 \\
\hline Eurasia & ZECK & 41.57 & 43.79 & 11.72 & 26.00 & 0.08 & 0.17 & 1.84 & 0.62 \\
\hline Eurasia & ARTU & 58.56 & 56.43 & 6.23 & 24.97 & 0.10 & 0.18 & 0.80 & -0.52 \\
\hline Eurasia & NVSK & 83.24 & 54.84 & -1.44 & 25.80 & 1.12 & 0.95 & 0.30 & -0.60 \\
\hline Eurasia & KSTU & 92.79 & 55.99 & -4.68 & 25.43 & 0.53 & 0.00 & -0.18 & -0.38 \\
\hline Eurasia & CASC & -9.42 & 38.69 & 16.78 & 17.85 & 0.08 & 0.09 & -0.09 & 0.05 \\
\hline Eurasia & TORS & -6.76 & 62.02 & 17.62 & 10.43 & 0.33 & 0.64 & 0.83 & -1.45 \\
\hline Eurasia & NEWL & -5.54 & 50.10 & 16.46 & 15.76 & 0.15 & 0.13 & -0.30 & 0.01 \\
\hline Eurasia & BRST & -4.50 & 48.38 & 16.82 & 16.77 & 0.13 & 0.09 & 0.10 & 0.33 \\
\hline Eurasia & MADR & -4.25 & 40.43 & 16.08 & 18.33 & 0.23 & 0.28 & -0.63 & -0.05 \\
\hline Eurasia & VILL & -3.95 & 40.44 & 16.41 & 18.64 & 0.14 & 0.00 & -0.29 & 0.20 \\
\hline Eurasia & CANT & -3.80 & 43.47 & 16.17 & 18.39 & 0.01 & 0.08 & -0.52 & 0.60 \\
\hline Eurasia & YEBE & -3.09 & 40.52 & 16.28 & 18.72 & 0.09 & 0.15 & -0.38 & 0.14 \\
\hline Eurasia & MORP & -1.69 & 55.21 & 16.92 & 15.35 & 0.23 & 0.24 & 0.35 & 0.12 \\
\hline Eurasia & NSTG & -1.44 & 55.01 & 16.18 & 17.30 & 0.13 & 0.59 & -0.38 & 1.96 \\
\hline Eurasia & HRM1 & -1.28 & 51.45 & 16.44 & 16.34 & 0.12 & 0.00 & -0.11 & -0.01 \\
\hline Eurasia & LROC & -1.22 & 46.16 & 16.26 & 18.09 & 0.38 & 0.10 & -0.30 & 0.41 \\
\hline
\end{tabular}


Table 15 - continued from previous page

\begin{tabular}{|c|c|c|c|c|c|c|c|c|c|}
\hline \multirow[b]{2}{*}{ Plate } & \multicolumn{3}{|c|}{ Station } & \multicolumn{4}{|c|}{ Horizontal Velocities (mm/yr) } & \multicolumn{2}{|c|}{ Residuals (mm/yr) } \\
\hline & ID & $\lambda(\mathrm{deg})$ & $\phi(\mathrm{deg})$ & $V_{N}$ & $V_{E}$ & $\sigma_{V_{N}}$ & $\sigma_{V_{E}}$ & $\mathrm{~N}$ & $\bar{E}$ \\
\hline Eurasia & ALAC & -0.48 & 38.34 & 16.74 & 21.43 & 0.15 & 0.59 & 0.22 & 1.94 \\
\hline Eurasia & CHIZ & -0.41 & 46.13 & 16.25 & 18.36 & 0.07 & 0.12 & -0.26 & 0.50 \\
\hline Eurasia & NPLD & -0.34 & 51.42 & 15.92 & 17.07 & 0.22 & 0.14 & -0.58 & 0.50 \\
\hline Eurasia & VALE & -0.34 & 39.48 & 16.09 & 19.82 & 0.09 & 0.13 & -0.41 & 0.52 \\
\hline India & MALD & 73.53 & 4.19 & 34.05 & 42.92 & 0.35 & 0.59 & 0.17 & -0.62 \\
\hline India & HYDE & 78.55 & 17.42 & 34.32 & 41.04 & 0.24 & 0.38 & 0.00 & -0.01 \\
\hline Nazca & EISL & -109.38 & -27.15 & -6.74 & 67.09 & 0.35 & 0.32 & -1.05 & -1.09 \\
\hline Nazca & GALA & -90.30 & -0.74 & 10.86 & 51.30 & 0.64 & 0.48 & -0.31 & -0.12 \\
\hline Nazca & GALA & -90.30 & -0.74 & 10.86 & 51.30 & 0.64 & 0.48 & -0.31 & -0.12 \\
\hline N. America & PUC1 & -110.81 & 39.60 & -8.30 & -14.13 & 0.09 & 0.11 & -0.03 & -0.18 \\
\hline N. America & NISU & -105.26 & 40.00 & -5.97 & -14.97 & 0.36 & 0.45 & 0.39 & -0.48 \\
\hline N. America & AMC2 & -104.52 & 38.80 & -5.69 & -14.39 & 0.11 & -0.00 & 0.41 & -0.14 \\
\hline N. America & MDO1 & -104.01 & 30.68 & -5.75 & -11.98 & 0.18 & 0.14 & 0.17 & 0.13 \\
\hline N. America & SUM1 & -102.51 & 34.83 & -6.00 & -13.06 & 1.36 & 0.28 & -0.61 & 0.27 \\
\hline N. America & AUS5 & -97.76 & 30.31 & -2.72 & -11.44 & 0.25 & 0.26 & 0.96 & 0.83 \\
\hline N. America & PATT & -95.72 & 31.78 & -2.54 & -12.75 & 0.10 & 0.19 & 0.40 & 0.01 \\
\hline N. America & ANG1 & -95.49 & 29.30 & -1.76 & -11.71 & 0.20 & 0.61 & 1.10 & 0.32 \\
\hline N. America & WNFL & -92.78 & 31.90 & -1.86 & -12.20 & 0.17 & 0.11 & 0.01 & 0.65 \\
\hline N. America & NLIB & -91.57 & 41.77 & -1.33 & -15.23 & 0.13 & 0.09 & 0.10 & 0.30 \\
\hline N. America & MIL1 & -87.89 & 43.00 & 0.10 & -14.97 & 0.04 & 0.25 & 0.18 & 0.90 \\
\hline N. America & MLF1 & -87.39 & 32.09 & 0.45 & -13.03 & 0.24 & 0.37 & 0.35 & -0.08 \\
\hline N. America & STB1 & -87.31 & 44.80 & -1.02 & -16.13 & 0.19 & 0.10 & -1.16 & 0.17 \\
\hline N. America & UNIV & -84.39 & 42.29 & 1.02 & -15.61 & 0.12 & 0.12 & -0.18 & 0.06 \\
\hline N. America & LEBA & -84.28 & 39.43 & 1.58 & -14.85 & 0.10 & 0.14 & 0.34 & 0.10 \\
\hline N. America & BAYR & -83.89 & 43.45 & 0.87 & -16.10 & 0.10 & 0.14 & -0.52 & -0.16 \\
\hline N. America & MCN1 & -83.56 & 32.70 & 1.76 & -13.23 & 0.16 & 0.14 & 0.25 & -0.14 \\
\hline N. America & ASHV & -82.55 & 35.60 & 2.15 & -14.13 & 0.18 & 0.15 & 0.27 & -0.24 \\
\hline N. America & MCD1 & -82.53 & 27.85 & 1.20 & -10.98 & 0.29 & 0.24 & -0.69 & 0.67 \\
\hline N. America & SAV1 & -81.70 & 32.14 & 2.42 & -12.62 & 0.12 & 0.13 & 0.23 & 0.29 \\
\hline
\end{tabular}


Table 15 - continued from previous page

\begin{tabular}{|c|c|c|c|c|c|c|c|c|c|}
\hline \multirow[b]{2}{*}{ Plate } & \multicolumn{3}{|c|}{ Station } & \multicolumn{4}{|c|}{ Horizontal Velocities $(\mathrm{mm} / \mathrm{yr})$} & \multicolumn{2}{|c|}{ Residuals (mm/yr) } \\
\hline & ID & $\lambda(\operatorname{deg})$ & $\phi(\mathrm{deg})$ & $V_{N}$ & $V_{E}$ & $\sigma_{V_{N}}$ & $\sigma_{V_{E}}$ & $\mathrm{~N}$ & $\mathrm{E}$ \\
\hline N. America & CCV3 & -80.55 & 28.46 & 2.98 & -12.48 & 0.26 & 0.31 & 0.37 & -0.69 \\
\hline N. America & CHA1 & -79.84 & 32.76 & 3.28 & -12.93 & 0.19 & 0.43 & 0.41 & 0.11 \\
\hline N. America & PSU1 & -77.85 & 40.81 & 3.66 & -15.18 & 0.17 & 0.08 & 0.07 & -0.06 \\
\hline N. America & GODE & -76.83 & 39.02 & 4.07 & -14.68 & 0.06 & 0.08 & 0.11 & -0.05 \\
\hline N. America & GLPT & -76.50 & 37.25 & 4.00 & -14.36 & 0.32 & 0.16 & -0.08 & -0.21 \\
\hline N. America & HNPT & -76.13 & 38.59 & 4.54 & -14.66 & 0.42 & 0.66 & 0.32 & -0.17 \\
\hline N. America & DUCK & -75.75 & 36.18 & 4.25 & -13.93 & 0.19 & 0.30 & -0.09 & -0.09 \\
\hline N. America & VIMS & -75.69 & 37.61 & 4.82 & -14.00 & 0.08 & 0.08 & 0.45 & 0.22 \\
\hline N. America & DNRC & -75.52 & 39.16 & 4.05 & -15.19 & 0.11 & 0.25 & -0.38 & -0.58 \\
\hline N. America & CHL1 & -75.09 & 38.78 & 4.02 & -14.63 & 0.19 & 0.20 & -0.57 & -0.15 \\
\hline N. America & WES2 & -71.49 & 42.61 & 5.40 & -15.12 & 0.14 & 0.07 & -0.46 & 0.09 \\
\hline N. America & NPRI & -71.33 & 41.51 & 5.67 & -15.09 & 0.10 & 0.11 & -0.25 & -0.15 \\
\hline N. America & BARH & -68.22 & 44.40 & 6.72 & -15.22 & 0.08 & 0.09 & -0.28 & 0.14 \\
\hline N. America & EPRT & -66.99 & 44.91 & 7.26 & -15.42 & 0.08 & 0.06 & -0.17 & -0.05 \\
\hline N. America & UNB1 & -66.64 & 45.95 & 7.13 & -15.78 & 0.42 & 0.40 & -0.42 & -0.22 \\
\hline N. America & BRMU & -64.70 & 32.37 & 8.85 & -11.81 & 0.20 & 0.37 & 0.63 & 0.33 \\
\hline N. America & HLFX & -63.61 & 44.68 & 8.67 & -15.22 & 0.10 & 0.16 & 0.09 & -0.23 \\
\hline Nubia & WIND & 17.09 & -22.57 & 19.98 & 18.73 & 0.37 & 0.19 & 0.72 & -1.26 \\
\hline Nubia & SIMO & 18.44 & -34.19 & 19.40 & 16.56 & 0.20 & 0.38 & 0.23 & -0.43 \\
\hline Nubia & SUTH & 20.81 & -32.38 & 19.11 & 16.76 & 0.11 & 0.18 & 0.10 & -0.29 \\
\hline Nubia & LPAL & -17.89 & 28.76 & 16.99 & 16.50 & 0.34 & 0.33 & -0.60 & 0.41 \\
\hline Nubia & DAKA & -17.47 & 14.68 & 14.12 & 21.38 & 0.58 & 0.85 & -3.54 & 1.27 \\
\hline Nubia & MAS1 & -15.63 & 27.76 & 17.63 & 16.65 & 0.16 & 0.17 & -0.28 & -0.08 \\
\hline Nubia & GOUG & -9.88 & -40.35 & 18.72 & 21.37 & 0.82 & 0.26 & 0.14 & 0.04 \\
\hline Pacific & MCIL & 153.98 & 24.29 & 24.19 & -71.71 & 0.30 & 0.37 & 0.57 & -0.05 \\
\hline Pacific & POHN & 158.21 & 6.96 & 25.46 & -70.19 & 0.30 & 0.41 & -0.01 & -0.77 \\
\hline Pacific & NAUR & 166.93 & -0.55 & 30.02 & -67.01 & 0.14 & 0.22 & 1.22 & -0.18 \\
\hline Pacific & KWJ1 & 167.73 & 8.72 & 29.17 & -68.59 & 0.35 & 0.74 & 0.09 & 0.59 \\
\hline Pacific & KIRI & 172.92 & 1.35 & 31.07 & -67.69 & 0.23 & 0.23 & 0.35 & -0.29 \\
\hline
\end{tabular}


Table 15 - continued from previous page

\begin{tabular}{|c|c|c|c|c|c|c|c|c|c|}
\hline \multirow[b]{2}{*}{ Plate } & \multicolumn{3}{|c|}{ Station } & \multicolumn{4}{|c|}{ Horizontal Velocities $(\mathrm{mm} / \mathrm{yr})$} & \multicolumn{2}{|c|}{ Residuals (mm/yr) } \\
\hline & ID & $\lambda(\mathrm{deg})$ & $\phi(\operatorname{deg})$ & $V_{N}$ & $V_{E}$ & $\sigma_{V_{N}}$ & $\sigma_{V_{E}}$ & $\mathrm{~N}$ & $\mathrm{E}$ \\
\hline Pacific & TUVA & 179.20 & -8.53 & 32.45 & -63.91 & 0.13 & 0.25 & 0.10 & 0.44 \\
\hline Pacific & CHAT & -176.57 & -43.96 & 33.21 & -40.59 & 0.13 & 0.15 & 0.02 & 0.29 \\
\hline Pacific & FALE & -172.00 & -13.83 & 33.26 & -63.48 & 0.08 & 0.23 & -0.74 & -0.31 \\
\hline Pacific & SAMO & -171.74 & -13.85 & 33.43 & -64.29 & 0.16 & 0.33 & -0.61 & -1.09 \\
\hline Pacific & ASPA & -170.72 & -14.33 & 34.15 & -63.16 & 0.11 & 0.27 & -0.02 & -0.01 \\
\hline Pacific & CKIS & -159.80 & -21.20 & 35.40 & -62.74 & 0.18 & 0.54 & 0.46 & -0.49 \\
\hline Pacific & KOK1 & -159.76 & 21.98 & 33.52 & -61.97 & 0.87 & 0.51 & -1.41 & 0.49 \\
\hline Pacific & KOKB & -159.66 & 22.13 & 34.63 & -62.28 & 0.13 & 0.20 & -0.31 & 0.11 \\
\hline Pacific & LHUE & -159.34 & 21.98 & 35.50 & -61.52 & 1.49 & 0.52 & 0.56 & 0.84 \\
\hline Pacific & HNLC & -157.86 & 21.30 & 34.84 & -62.52 & 0.41 & 0.17 & -0.10 & -0.20 \\
\hline Pacific & UPO1 & -155.88 & 20.25 & 35.69 & -67.78 & 0.51 & 0.80 & 0.79 & -5.43 \\
\hline Pacific & MKEA & -155.46 & 19.80 & 34.86 & -62.19 & 0.10 & 0.15 & -0.06 & 0.30 \\
\hline Pacific & HILO & -155.05 & 19.72 & 38.72 & -62.45 & 0.68 & 0.46 & 3.84 & -0.04 \\
\hline Pacific & THTI & -149.61 & -17.58 & 34.42 & -65.40 & 0.18 & 0.29 & -0.10 & 0.16 \\
\hline Pacific & GUAX & -118.29 & 28.88 & 26.05 & -47.68 & 0.74 & 0.69 & -0.60 & 0.20 \\
\hline S. America & BUE2 & -58.52 & -34.57 & 12.48 & -0.65 & 1.51 & 0.23 & 1.05 & 1.17 \\
\hline S. America & LPGS & -57.93 & -34.91 & 11.76 & -0.95 & 0.10 & 0.13 & 0.29 & 0.90 \\
\hline S. America & LKTH & -57.85 & -51.70 & 12.33 & 0.34 & 0.51 & 0.38 & 0.86 & 0.32 \\
\hline S. America & KOUR & -52.81 & 5.25 & 12.62 & -5.21 & 0.12 & 0.08 & 0.81 & 0.14 \\
\hline S. America & UEPP & -51.41 & -22.12 & 12.76 & -3.22 & 0.35 & 0.24 & 0.89 & 0.47 \\
\hline S. America & PARA & -49.23 & -25.45 & 12.16 & -3.54 & 0.19 & 0.34 & 0.19 & 0.08 \\
\hline S. America & NEIA & -47.92 & -25.02 & 12.71 & -2.38 & 0.24 & 0.20 & 0.69 & 1.39 \\
\hline S. America & BRAZ & -47.88 & -15.95 & 12.61 & -4.22 & 0.13 & 0.17 & 0.59 & 0.13 \\
\hline S. America & FORT & -38.43 & -3.88 & 12.35 & -4.21 & 0.16 & 0.30 & 0.15 & 0.88 \\
\hline S. America & ASC1 & -14.41 & -7.95 & 11.15 & -5.21 & 0.20 & 0.26 & 0.01 & 0.51 \\
\hline Somalia & MALI & 40.19 & -3.00 & 16.31 & 26.78 & 0.20 & 0.26 & -0.65 & 1.83 \\
\hline Somalia & REUN & 55.57 & -21.21 & 12.51 & 16.59 & 0.18 & 0.31 & -0.99 & -1.89 \\
\hline Sunda & NTUS & 103.68 & 1.35 & -5.24 & 30.76 & 0.14 & 0.18 & -0.00 & 0.01 \\
\hline
\end{tabular}


Table 16: Station Information and Velocity Estimates from the CATS method.

\begin{tabular}{|c|c|c|c|c|c|c|c|c|c|}
\hline \multirow[b]{2}{*}{ Plate } & \multicolumn{3}{|c|}{ Station } & \multicolumn{4}{|c|}{ Horizontal Velocities (mm/yr) } & \multicolumn{2}{|c|}{ Residuals (mm/yr) } \\
\hline & ID & $\lambda(\mathrm{deg})$ & $\phi(\mathrm{deg})$ & $V_{N}$ & $V_{E}$ & $\sigma_{V_{N}}$ & $\sigma_{V_{E}}$ & $\mathrm{~N}$ & $\mathrm{E}$ \\
\hline Amurian & CHAN & 125.44 & 43.79 & -11.67 & 26.12 & 0.56 & 0.19 & 2.43 & 1.24 \\
\hline Amurian & KHAJ & 135.05 & 48.52 & -13.62 & 21.66 & 0.22 & 0.09 & 1.14 & 0.04 \\
\hline Antartica & SYOG & 39.58 & -69.01 & 2.90 & -4.05 & 0.06 & 0.06 & -0.02 & -0.04 \\
\hline Antartica & DAV1 & 77.97 & -68.58 & -5.26 & -3.06 & 0.06 & 0.13 & -0.25 & 0.10 \\
\hline Antartica & CAS1 & 110.52 & -66.28 & -9.99 & 1.85 & 0.08 & 0.11 & 0.16 & 0.08 \\
\hline Antartica & DUM1 & 140.00 & -66.67 & -11.41 & 8.52 & 0.32 & 1.04 & 0.67 & 1.52 \\
\hline Antartica & VESL & -2.84 & -71.67 & 10.27 & -0.25 & 0.08 & 0.07 & 0.19 & 0.00 \\
\hline Arabia & HALY & 36.10 & 29.14 & 22.96 & 26.81 & 0.17 & 0.28 & -2.07 & -0.04 \\
\hline Arabia & BHR2 & 50.61 & 26.21 & 30.25 & 31.25 & 0.08 & 0.13 & 1.57 & -0.37 \\
\hline Arabia & YIBL & 56.11 & 22.19 & 31.37 & 33.40 & 0.11 & 0.26 & 1.77 & -1.12 \\
\hline Australia & YAR1 & 115.35 & -29.05 & 57.31 & 39.12 & 0.24 & 0.25 & -0.87 & -0.13 \\
\hline Australia & NNOR & 116.19 & -31.05 & 57.92 & 38.41 & 0.07 & 0.09 & -0.43 & -0.12 \\
\hline Australia & KARR & 117.10 & -20.98 & 58.38 & 38.92 & 0.08 & 0.06 & -0.18 & -0.32 \\
\hline Australia & DARW & 131.13 & -12.84 & 59.22 & 35.91 & 0.10 & 0.30 & -0.25 & -0.20 \\
\hline Australia & CEDU & 133.81 & -31.87 & 58.78 & 29.11 & 0.09 & 0.10 & -0.41 & 0.28 \\
\hline Australia & ALIC & 133.89 & -23.67 & 59.10 & 32.10 & 0.06 & 0.07 & -0.11 & -0.00 \\
\hline Australia & ADE1 & 138.65 & -34.73 & 58.38 & 24.95 & 0.11 & 0.13 & -0.05 & 0.24 \\
\hline Australia & TOW2 & 147.06 & -19.27 & 55.73 & 28.85 & 0.06 & 0.06 & -0.46 & -0.41 \\
\hline Australia & HOB2 & 147.44 & -42.80 & 55.63 & 14.18 & 0.74 & 0.08 & -0.36 & -0.00 \\
\hline Australia & PARK & 148.26 & -33.00 & 53.07 & 18.92 & 0.31 & 0.30 & -2.65 & -1.50 \\
\hline Australia & TIDB & 148.98 & -35.40 & 55.25 & 18.29 & 0.06 & 0.07 & -0.20 & -0.12 \\
\hline Australia & STR1 & 149.01 & -35.32 & 55.35 & 18.65 & 0.08 & 0.07 & -0.10 & 0.20 \\
\hline Australia & SYDN & 151.15 & -33.78 & 54.35 & 18.06 & 0.15 & 0.15 & -0.25 & -0.30 \\
\hline Australia & SUNM & 153.04 & -27.48 & 53.95 & 21.95 & 0.24 & 0.20 & 0.15 & 0.06 \\
\hline Australia & KOUC & 164.29 & -20.56 & 47.58 & 22.47 & 0.17 & 0.25 & -0.22 & -0.58 \\
\hline Australia & NOUM & 166.41 & -22.27 & 45.79 & 20.57 & 0.14 & 0.14 & -0.66 & -0.43 \\
\hline Australia & AUCK & 174.83 & -36.60 & 39.74 & 4.50 & 0.07 & 0.11 & -0.72 & -0.00 \\
\hline
\end{tabular}


Table 16 - continued from previous page

\begin{tabular}{|c|c|c|c|c|c|c|c|c|c|}
\hline \multirow[b]{2}{*}{ Plate } & \multicolumn{3}{|c|}{ Station } & \multicolumn{4}{|c|}{ Horizontal Velocities (mm/yr) } & \multicolumn{2}{|c|}{ Residuals (mm/yr) } \\
\hline & ID & $\lambda(\mathrm{deg})$ & $\phi(\mathrm{deg})$ & $V_{N}$ & $V_{E}$ & $\sigma_{V_{N}}$ & $\sigma_{V_{E}}$ & $\mathrm{~N}$ & $\mathrm{E}$ \\
\hline Caribbean & CRO1 & -64.58 & 17.76 & 13.54 & 11.04 & 0.08 & 0.19 & 0.06 & -0.83 \\
\hline Eurasia & HERS & 0.34 & 50.87 & 16.47 & 16.50 & 0.25 & 0.13 & -0.20 & -0.51 \\
\hline Eurasia & EBRE & 0.49 & 40.82 & 15.82 & 19.84 & 0.12 & 0.04 & -0.85 & 0.25 \\
\hline Eurasia & SHEE & 0.74 & 51.45 & 16.57 & 16.70 & 0.27 & 0.08 & -0.08 & -0.24 \\
\hline Eurasia & BELL & 1.40 & 41.60 & 15.96 & 19.56 & 0.17 & 0.09 & -0.66 & -0.03 \\
\hline Eurasia & TOUL & 1.48 & 43.56 & 16.81 & 20.06 & 0.25 & 0.27 & 0.20 & 0.91 \\
\hline Eurasia & OPMT & 2.33 & 48.84 & 15.79 & 18.15 & 0.09 & 0.06 & -0.76 & 0.14 \\
\hline Eurasia & MALL & 2.62 & 39.55 & 16.29 & 19.80 & 0.09 & 0.15 & -0.25 & -0.46 \\
\hline Eurasia & SJDV & 4.68 & 45.88 & 16.05 & 19.45 & 0.08 & 0.06 & -0.35 & 0.20 \\
\hline Eurasia & REDU & 5.14 & 50.00 & 15.59 & 18.28 & 0.09 & 0.11 & -0.77 & -0.03 \\
\hline Eurasia & MARS & 5.35 & 43.28 & 15.96 & 20.05 & 0.06 & 0.06 & -0.39 & 0.07 \\
\hline Eurasia & KOSG & 5.81 & 52.18 & 16.01 & 18.05 & 0.08 & 0.06 & -0.29 & 0.16 \\
\hline Eurasia & WSRT & 6.60 & 52.91 & 16.41 & 17.64 & 0.07 & 0.05 & 0.17 & -0.22 \\
\hline Eurasia & BORK & 6.75 & 53.56 & 15.22 & 17.59 & 0.18 & 0.08 & -1.01 & -0.13 \\
\hline Eurasia & WAB2 & 7.46 & 46.92 & 15.84 & 19.85 & 0.08 & 0.09 & -0.33 & 0.28 \\
\hline Eurasia & ZIMM & 7.47 & 46.88 & 16.22 & 19.60 & 0.07 & 0.04 & 0.05 & 0.01 \\
\hline Eurasia & IENG & 7.64 & 45.02 & 15.40 & 20.48 & 0.09 & 0.09 & -0.75 & 0.43 \\
\hline Eurasia & HELG & 7.89 & 54.17 & 15.93 & 17.63 & 0.09 & 0.07 & -0.19 & -0.18 \\
\hline Eurasia & AJAC & 8.76 & 41.93 & 15.75 & 21.35 & 0.10 & 0.21 & -0.30 & 0.44 \\
\hline Eurasia & PTBB & 10.46 & 52.30 & 15.55 & 18.81 & 0.07 & 0.06 & -0.33 & -0.07 \\
\hline Eurasia & WARN & 12.10 & 54.17 & 15.58 & 18.32 & 0.09 & 0.06 & -0.12 & -0.44 \\
\hline Eurasia & BUDP & 12.50 & 55.74 & 14.94 & 18.01 & 0.07 & 0.05 & -0.71 & -0.41 \\
\hline Eurasia & WTZR & 12.88 & 49.14 & 15.55 & 20.39 & 0.06 & 0.04 & -0.07 & 0.22 \\
\hline Eurasia & POTS & 13.07 & 52.38 & 15.12 & 19.16 & 0.08 & 0.05 & -0.47 & -0.27 \\
\hline Eurasia & SASS & 13.64 & 54.51 & 14.66 & 19.00 & 0.16 & 0.06 & -0.87 & -0.00 \\
\hline Eurasia & GOPE & 14.79 & 49.91 & 15.10 & 19.99 & 0.13 & 0.10 & -0.29 & -0.40 \\
\hline Eurasia & GRAZ & 15.49 & 47.07 & 15.38 & 21.93 & 0.07 & 0.06 & 0.08 & 0.78 \\
\hline Eurasia & WROC & 17.06 & 51.11 & 14.63 & 20.17 & 0.09 & 0.06 & -0.47 & -0.40 \\
\hline Eurasia & BOR1 & 17.07 & 52.28 & 14.58 & 20.01 & 0.06 & 0.07 & -0.51 & -0.29 \\
\hline
\end{tabular}


Table 16 - continued from previous page

\begin{tabular}{|c|c|c|c|c|c|c|c|c|c|}
\hline \multirow[b]{2}{*}{ Plate } & \multicolumn{3}{|c|}{ Station } & \multicolumn{4}{|c|}{ Horizontal Velocities $(\mathrm{mm} / \mathrm{yr})$} & \multicolumn{2}{|c|}{ Residuals (mm/yr) } \\
\hline & ID & $\lambda(\operatorname{deg})$ & $\phi(\mathrm{deg})$ & $\overline{V_{N}}$ & $V_{E}$ & $\sigma_{V_{N}}$ & $\sigma_{V_{E}}$ & $\mathrm{~N}$ & $\mathrm{E}$ \\
\hline Eurasia & PENC & 19.28 & 47.79 & 14.66 & 22.15 & 0.13 & 0.05 & -0.13 & 0.41 \\
\hline Eurasia & LAMA & 20.67 & 53.89 & 14.36 & 20.10 & 0.15 & 0.05 & -0.22 & -0.56 \\
\hline Eurasia & JOZE & 21.03 & 52.10 & 14.38 & 21.03 & 0.09 & 0.07 & -0.14 & -0.12 \\
\hline Eurasia & BOGO & 21.04 & 52.48 & 14.44 & 20.57 & 0.12 & 0.10 & -0.09 & -0.49 \\
\hline Eurasia & KLPD & 21.12 & 55.72 & 13.43 & 20.06 & 0.24 & 0.30 & -1.08 & -0.25 \\
\hline Eurasia & UZHL & 22.30 & 48.63 & 13.89 & 21.83 & 0.08 & 0.10 & -0.44 & -0.30 \\
\hline Eurasia & SULP & 24.01 & 49.84 & 13.95 & 21.47 & 0.10 & 0.09 & -0.10 & -0.74 \\
\hline Eurasia & RIGA & 24.06 & 56.95 & 13.44 & 20.21 & 0.07 & 0.08 & -0.60 & -0.40 \\
\hline Eurasia & GLSV & 30.50 & 50.36 & 12.84 & 22.37 & 0.09 & 0.07 & -0.06 & -0.90 \\
\hline Eurasia & MIKL & 31.97 & 46.97 & 12.07 & 23.52 & 0.12 & 0.11 & -0.55 & -0.60 \\
\hline Eurasia & CRAO & 33.99 & 44.41 & 11.50 & 23.73 & 0.16 & 0.29 & -0.71 & -1.08 \\
\hline Eurasia & KHAR & 36.24 & 50.01 & 11.91 & 24.20 & 0.17 & 0.17 & 0.18 & -0.09 \\
\hline Eurasia & MOBN & 36.57 & 55.11 & 11.74 & 22.77 & 0.38 & 0.16 & 0.07 & -0.59 \\
\hline Eurasia & ZECK & 41.57 & 43.79 & 11.72 & 26.02 & 0.06 & 0.12 & 1.16 & 0.08 \\
\hline Eurasia & ARTU & 58.56 & 56.43 & 6.23 & 24.98 & 0.08 & 0.12 & 0.02 & -0.99 \\
\hline Eurasia & NVSK & 83.24 & 54.84 & -0.93 & 25.78 & 1.12 & 0.79 & -0.00 & -1.42 \\
\hline Eurasia & KSTU & 92.79 & 55.99 & -4.53 & 25.68 & 0.36 & 0.25 & -0.81 & -1.00 \\
\hline Eurasia & CASC & -9.42 & 38.69 & 16.79 & 17.85 & 0.05 & 0.07 & -0.16 & -0.39 \\
\hline Eurasia & TORS & -6.76 & 62.02 & 17.57 & 10.63 & 0.23 & 0.60 & 0.67 & -1.11 \\
\hline Eurasia & NEWL & -5.54 & 50.10 & 16.36 & 15.75 & 0.17 & 0.06 & -0.53 & -0.16 \\
\hline Eurasia & BRST & -4.50 & 48.38 & 16.84 & 16.77 & 0.09 & 0.07 & -0.02 & 0.12 \\
\hline Eurasia & MADR & -4.25 & 40.43 & 16.03 & 18.38 & 0.17 & 0.20 & -0.84 & -0.41 \\
\hline Eurasia & VILL & -3.95 & 40.44 & 16.42 & 18.73 & 0.12 & 0.08 & -0.43 & -0.11 \\
\hline Eurasia & CANT & -3.80 & 43.47 & 16.45 & 18.41 & 0.07 & 0.06 & -0.40 & 0.29 \\
\hline Eurasia & YEBE & -3.09 & 40.52 & 16.29 & 18.72 & 0.05 & 0.12 & -0.54 & -0.26 \\
\hline Eurasia & MORP & -1.69 & 55.21 & 16.79 & 15.30 & 0.19 & 0.26 & 0.03 & 0.04 \\
\hline Eurasia & NSTG & -1.44 & 55.01 & 16.24 & 16.25 & 0.07 & 0.19 & -0.51 & 0.87 \\
\hline Eurasia & HRM1 & -1.28 & 51.45 & 16.44 & 16.41 & 0.08 & 0.05 & -0.30 & -0.08 \\
\hline Eurasia & LROC & -1.22 & 46.16 & 16.32 & 18.10 & 0.06 & 0.06 & -0.43 & 0.15 \\
\hline
\end{tabular}


Table 16 - continued from previous page

\begin{tabular}{|c|c|c|c|c|c|c|c|c|c|}
\hline \multirow[b]{2}{*}{ Plate } & \multicolumn{3}{|c|}{ Station } & \multicolumn{4}{|c|}{ Horizontal Velocities (mm/yr) } & \multicolumn{2}{|c|}{ Residuals (mm/yr) } \\
\hline & ID & $\lambda(\mathrm{deg})$ & $\phi(\mathrm{deg})$ & $V_{N}$ & $V_{E}$ & $\sigma_{V_{N}}$ & $\sigma_{V_{E}}$ & $\mathrm{~N}$ & $\mathrm{E}$ \\
\hline Eurasia & ALAC & -0.48 & 38.34 & 16.67 & 20.21 & 0.07 & 0.13 & -0.05 & 0.26 \\
\hline Eurasia & CHIZ & -0.41 & 46.13 & 16.25 & 18.35 & 0.05 & 0.09 & -0.46 & 0.22 \\
\hline Eurasia & NPLD & -0.34 & 51.42 & 15.94 & 17.03 & 0.14 & 0.08 & -0.76 & 0.32 \\
\hline Eurasia & VALE & -0.34 & 39.48 & 16.09 & 19.75 & 0.06 & 0.09 & -0.62 & 0.01 \\
\hline India & MALD & 73.53 & 4.19 & 34.04 & 43.30 & 0.24 & 0.49 & 0.25 & -0.61 \\
\hline India & HYDE & 78.55 & 17.42 & 34.25 & 41.06 & 0.24 & 0.17 & -0.11 & -0.02 \\
\hline Nazca & EISL & -109.38 & -27.15 & -6.78 & 67.07 & 0.28 & 0.29 & -0.70 & -0.77 \\
\hline Nazca & GALA & -90.30 & -0.74 & 10.45 & 51.37 & 0.17 & 0.13 & -0.10 & -0.04 \\
\hline Nazca & GALA & -90.30 & -0.74 & 10.45 & 51.37 & 0.17 & 0.13 & -0.10 & -0.04 \\
\hline N. America & PUC1 & -110.81 & 39.60 & -8.30 & -14.12 & 0.07 & 0.09 & -1.21 & 0.12 \\
\hline N. America & NISU & -105.26 & 40.00 & -5.98 & -15.00 & 0.24 & 0.18 & -0.64 & -0.29 \\
\hline N. America & $\mathrm{AMC} 2$ & -104.52 & 38.80 & -5.69 & -14.45 & 0.08 & 0.10 & -0.59 & 0.05 \\
\hline N. America & MDO1 & -104.01 & 30.68 & -5.81 & -12.01 & 0.10 & 0.10 & -0.87 & 0.68 \\
\hline N. America & SUM1 & -102.51 & 34.83 & -5.76 & -13.06 & 0.80 & 0.21 & -1.31 & 0.67 \\
\hline N. America & AUS5 & -97.76 & 30.31 & -2.74 & -11.46 & 0.18 & 0.19 & 0.15 & 1.35 \\
\hline N. America & PATT & -95.72 & 31.78 & -2.55 & -12.73 & 0.08 & 0.13 & -0.33 & 0.49 \\
\hline N. America & ANG1 & -95.49 & 29.30 & -1.78 & -11.73 & 0.13 & 0.38 & 0.36 & 0.88 \\
\hline N. America & WNFL & -92.78 & 31.90 & -1.85 & -12.20 & 0.14 & 0.08 & -0.61 & 1.10 \\
\hline N. America & NLIB & -91.57 & 41.77 & -1.30 & -15.24 & 0.11 & 0.08 & -0.46 & 0.31 \\
\hline N. America & MIL1 & -87.89 & 43.00 & -0.06 & -15.04 & 0.17 & 0.19 & -0.45 & 0.76 \\
\hline N. America & MLF1 & -87.39 & 32.09 & 0.37 & -12.97 & 0.19 & 0.26 & -0.18 & 0.39 \\
\hline N. America & STB1 & -87.31 & 44.80 & -0.93 & -16.12 & 0.15 & 0.08 & -1.51 & 0.03 \\
\hline N. America & UNIV & -84.39 & 42.29 & 1.03 & -15.61 & 0.08 & 0.09 & -0.52 & 0.01 \\
\hline N. America & LEBA & -84.28 & 39.43 & 1.59 & -14.85 & 0.08 & 0.10 & 0.00 & 0.17 \\
\hline N. America & BAYR & -83.89 & 43.45 & 0.88 & -16.11 & 0.08 & 0.09 & -0.84 & -0.27 \\
\hline N. America & MCN1 & -83.56 & 32.70 & 1.78 & -13.23 & 0.12 & 0.10 & -0.05 & 0.24 \\
\hline N. America & ASHV & -82.55 & 35.60 & 2.09 & -14.14 & 0.12 & 0.11 & -0.08 & -0.00 \\
\hline N. America & MCD1 & -82.53 & 27.85 & 1.26 & -10.99 & 0.20 & 0.17 & -0.91 & 1.24 \\
\hline N. America & SAV1 & -81.70 & 32.14 & 2.39 & -12.63 & 0.08 & 0.11 & -0.06 & 0.66 \\
\hline
\end{tabular}


Table 16 - continued from previous page

\begin{tabular}{|c|c|c|c|c|c|c|c|c|c|}
\hline \multirow[b]{2}{*}{ Plate } & \multicolumn{3}{|c|}{ Station } & \multicolumn{4}{|c|}{ Horizontal Velocities $(\mathrm{mm} / \mathrm{yr})$} & \multicolumn{2}{|c|}{ Residuals (mm/yr) } \\
\hline & ID & $\lambda(\mathrm{deg})$ & $\phi(\mathrm{deg})$ & $V_{N}$ & $V_{E}$ & $\sigma_{V_{N}}$ & $\sigma_{V_{E}}$ & $\mathrm{~N}$ & $\mathrm{E}$ \\
\hline N. America & $\mathrm{CCV} 3$ & -80.55 & 28.46 & 2.88 & -12.30 & 0.14 & 0.10 & 0.05 & 0.05 \\
\hline N. America & CHA1 & -79.84 & 32.76 & 3.28 & -12.90 & 0.17 & 0.19 & 0.22 & 0.50 \\
\hline N. America & PSU1 & -77.85 & 40.81 & 3.68 & -15.19 & 0.15 & 0.06 & -0.04 & -0.07 \\
\hline N. America & GODE & -76.83 & 39.02 & 4.07 & -14.68 & 0.06 & 0.07 & 0.02 & 0.03 \\
\hline N. America & GLPT & -76.50 & 37.25 & 4.08 & -14.35 & 0.06 & 0.12 & -0.08 & -0.04 \\
\hline N. America & HNPT & -76.13 & 38.59 & 4.54 & -14.44 & 0.37 & 0.54 & 0.26 & 0.14 \\
\hline N. America & DUCK & -75.75 & 36.18 & 4.24 & -13.96 & 0.15 & 0.22 & -0.16 & 0.08 \\
\hline N. America & VIMS & -75.69 & 37.61 & 4.75 & -14.06 & 0.08 & 0.05 & 0.33 & 0.29 \\
\hline N. America & DNRC & -75.52 & 39.16 & 4.02 & -15.17 & 0.09 & 0.20 & -0.46 & -0.49 \\
\hline N. America & CHL1 & -75.09 & 38.78 & 3.99 & -14.61 & 0.15 & 0.13 & -0.63 & -0.04 \\
\hline N. America & WES2 & -71.49 & 42.61 & 5.41 & -15.12 & 0.12 & 0.07 & -0.36 & 0.01 \\
\hline N. America & NPRI & -71.33 & 41.51 & 5.66 & -15.06 & 0.07 & 0.06 & -0.16 & -0.16 \\
\hline N. America & BARH & -68.22 & 44.40 & 6.73 & -15.23 & 0.06 & 0.05 & -0.07 & -0.02 \\
\hline N. America & EPRT & -66.99 & 44.91 & 7.25 & -15.42 & 0.07 & 0.04 & 0.07 & -0.23 \\
\hline N. America & UNB1 & -66.64 & 45.95 & 7.30 & -15.87 & 0.24 & 0.27 & 0.01 & -0.54 \\
\hline N. America & BRMU & -64.70 & 32.37 & 8.87 & -11.97 & 0.18 & 0.38 & 0.98 & 0.56 \\
\hline N. America & HLFX & -63.61 & 44.68 & 8.67 & -15.21 & 0.06 & 0.05 & 0.45 & -0.38 \\
\hline Nubia & WIND & 17.09 & -22.57 & 19.54 & 19.00 & 0.11 & 0.14 & 0.30 & -1.05 \\
\hline Nubia & SIMO & 18.44 & -34.19 & 19.43 & 16.68 & 0.14 & 0.31 & 0.29 & -0.33 \\
\hline Nubia & SUTH & 20.81 & -32.38 & 19.10 & 16.86 & 0.09 & 0.11 & 0.11 & -0.23 \\
\hline Nubia & LPAL & -17.89 & 28.76 & 17.17 & 16.13 & 0.11 & 0.09 & -0.44 & -0.06 \\
\hline Nubia & DAKA & -17.47 & 14.68 & 14.16 & 21.02 & 0.43 & 0.39 & -3.52 & 0.82 \\
\hline Nubia & MAS1 & -15.63 & 27.76 & 17.57 & 16.62 & 0.15 & 0.13 & -0.36 & -0.22 \\
\hline Nubia & GOUG & -9.88 & -40.35 & 18.80 & 21.36 & 0.20 & 0.19 & 0.21 & 0.02 \\
\hline Pacific & MCIL & 153.98 & 24.29 & 24.13 & -71.71 & 0.21 & 0.12 & -1.40 & -1.17 \\
\hline Pacific & POHN & 158.21 & 6.96 & 25.61 & -70.10 & 0.20 & 0.11 & -1.62 & -1.30 \\
\hline Pacific & NAUR & 166.93 & -0.55 & 30.05 & -67.00 & 0.10 & 0.10 & -0.20 & -0.53 \\
\hline Pacific & KWJ1 & 167.73 & 8.72 & 29.32 & -68.90 & 0.12 & 0.12 & -1.17 & -0.45 \\
\hline Pacific & KIRI & 172.92 & 1.35 & 31.09 & -67.75 & 0.15 & 0.08 & -0.82 & -0.79 \\
\hline
\end{tabular}


Table 16 - continued from previous page

\begin{tabular}{|c|c|c|c|c|c|c|c|c|c|}
\hline \multirow[b]{2}{*}{ Plate } & \multicolumn{3}{|c|}{ Station } & \multicolumn{4}{|c|}{ Horizontal Velocities (mm/yr) } & \multicolumn{2}{|c|}{ Residuals (mm/yr) } \\
\hline & ID & $\lambda(\mathrm{deg})$ & $\phi(\mathrm{deg})$ & $V_{N}$ & $V_{E}$ & $\sigma_{V_{N}}$ & $\sigma_{V_{E}}$ & $\mathrm{~N}$ & $\bar{E}$ \\
\hline Pacific & TUVA & 179.20 & -8.53 & 32.43 & -63.88 & 0.06 & 0.13 & -0.85 & 0.44 \\
\hline Pacific & CHAT & -176.57 & -43.96 & 33.21 & -40.58 & 0.08 & 0.11 & -0.73 & 1.77 \\
\hline Pacific & FALE & -172.00 & -13.83 & 33.26 & -63.55 & 0.06 & 0.12 & -1.27 & -0.14 \\
\hline Pacific & SAMO & -171.74 & -13.85 & 33.47 & -64.09 & 0.09 & 0.23 & -1.09 & -0.65 \\
\hline Pacific & ASPA & -170.72 & -14.33 & 34.16 & -63.24 & 0.07 & 0.16 & -0.49 & 0.17 \\
\hline Pacific & CKIS & -159.80 & -21.20 & 35.35 & -62.49 & 0.11 & 0.13 & 0.45 & 0.35 \\
\hline Pacific & KOK1 & -159.76 & 21.98 & 35.13 & -62.21 & 0.11 & 0.13 & 0.22 & -1.11 \\
\hline Pacific & KOKB & -159.66 & 22.13 & 34.64 & -62.24 & 0.07 & 0.12 & -0.26 & -1.21 \\
\hline Pacific & LHUE & -159.34 & 21.98 & 34.93 & -61.45 & 1.71 & 0.29 & 0.04 & -0.44 \\
\hline Pacific & HNLC & -157.86 & 21.30 & 34.62 & -62.54 & 0.05 & 0.06 & -0.20 & -1.54 \\
\hline Pacific & UPO1 & -155.88 & 20.25 & 35.39 & -67.33 & 0.33 & 0.49 & 0.69 & -6.26 \\
\hline Pacific & MKEA & -155.46 & 19.80 & 34.85 & -62.23 & 0.06 & 0.08 & 0.17 & -1.00 \\
\hline Pacific & HILO & -155.05 & 19.72 & 35.44 & -63.02 & 0.07 & 0.38 & 0.81 & -1.88 \\
\hline Pacific & THTI & -149.61 & -17.58 & 34.46 & -65.44 & 0.08 & 0.11 & 0.44 & 0.53 \\
\hline Pacific & GUAX & -118.29 & 28.88 & 25.33 & -46.99 & 0.20 & 0.22 & 0.47 & -0.40 \\
\hline S. America & BUE2 & -58.52 & -34.57 & 12.46 & 0.00 & 0.99 & 0.01 & 1.21 & 1.91 \\
\hline S. America & LPGS & -57.93 & -34.91 & 11.77 & -0.95 & 0.09 & 0.10 & 0.48 & 0.98 \\
\hline S. America & LKTH & -57.85 & -51.70 & 12.29 & 0.38 & 0.17 & 0.20 & 1.00 & 0.38 \\
\hline S. America & KOUR & -52.81 & 5.25 & 12.63 & -5.21 & 0.10 & 0.07 & 1.00 & 0.31 \\
\hline S. America & UEPP & -51.41 & -22.12 & 12.82 & -3.29 & 0.18 & 0.24 & 1.12 & 0.50 \\
\hline S. America & PARA & -49.23 & -25.45 & 12.18 & -3.53 & 0.14 & 0.26 & 0.38 & 0.18 \\
\hline S. America & NEIA & -47.92 & -25.02 & 12.74 & -2.45 & 0.19 & 0.15 & 0.89 & 1.41 \\
\hline S. America & BRAZ & -47.88 & -15.95 & 12.59 & -4.29 & 0.11 & 0.13 & 0.73 & 0.18 \\
\hline S. America & FORT & -38.43 & -3.88 & 12.33 & -4.26 & 0.14 & 0.27 & 0.27 & 0.97 \\
\hline S. America & ASC1 & -14.41 & -7.95 & 11.04 & -5.24 & 0.11 & 0.16 & -0.04 & 0.62 \\
\hline Somalia & MALI & 40.19 & -3.00 & 16.34 & 26.75 & 0.14 & 0.20 & -0.31 & 1.75 \\
\hline Somalia & REUN & 55.57 & -21.21 & 12.43 & 16.89 & 0.10 & 0.20 & -0.97 & -1.90 \\
\hline Sunda & NTUS & 103.68 & 1.35 & -5.26 & 30.27 & 0.18 & 0.24 & 0.01 & 0.28 \\
\hline
\end{tabular}




\section{B An Evaluation of the MCMC using Hector and the Benchmark Synthetic GNSS time series}

Here we provide an evaluation of the MCMC method as implemented by Olivares and Teferle (2013) using Hector and the Benchmark Synthetic GNSS (BSG) time series. While providing the results from MCMC by themselves, we also carry out a basic comparison with the results provided and computed in-house with Hector. We have computed our own Hector parameter estimates since MCMC is a computationally intensive method and we have down-sampled the daily time series into weekly ones for all BSG series, see Fig. 36 for an example showing both original and downsampled time series. Fig. 37 shows the differences in the trend estimates $v$ between the two methods. Overall the parameter estimates (trend, amplitude of the annual term and phase-lag, white and power-low noise amplitudes as well as spectral index) are in good agreement between MCMC and Hector. A detailed comparison is shown in Tables 17, 18, and 19 for the deterministic parameter estimates, and in Tables 20, 21 and 22 for the stochastic parameter estimates.

\section{B.1 Gaussian properties of parameters estimates from MCMC}

The parameters estimated from MCMC follow in general a Gaussian distribution. While these histograms can provide valuable additional information it is clear that in several cases the MCMC method has failed to provide converged results. This is most likely due to instabilities in the variance/covariance matrix within the MCMC method.. Fig. 38 and 39 show the histograms for the trend components for the 20 time series.

Further we have tested the Gaussian properties of the parameter estimates from the MCMC method by constructing the histograms for the amplitude estimates of the annual terms as shown in Figures 40, 41, 42, 43, 44, and 45 for the North, East and Up components, respectively.

We have also compared the mean and median trend estimates for all the time series considered in this analysis. The mean and median values show a similar magnitude. An indication that the estimates from MCMC exhibit unbiased and uncorrelated properties. A further test for the Skewness and the Kurtosis ("tailedness" of the probability distribution) for the trends again show the majority of the estimates indeed follow a Gaussian distribution, see Tables 23, 24, and 25. Formally, the Skewness of a Gaussian distribution is 0 while the Kurtossis is 3. 


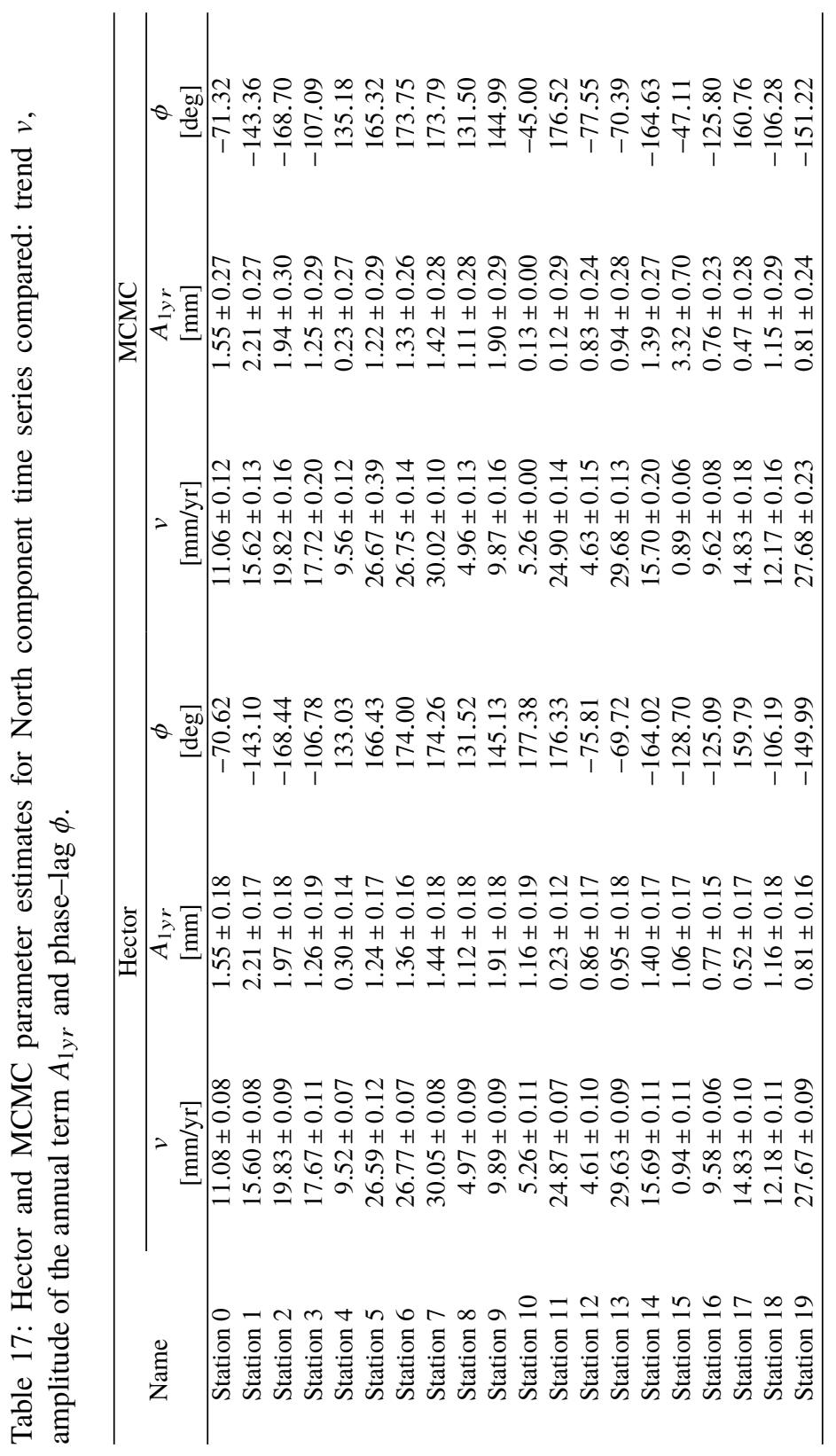




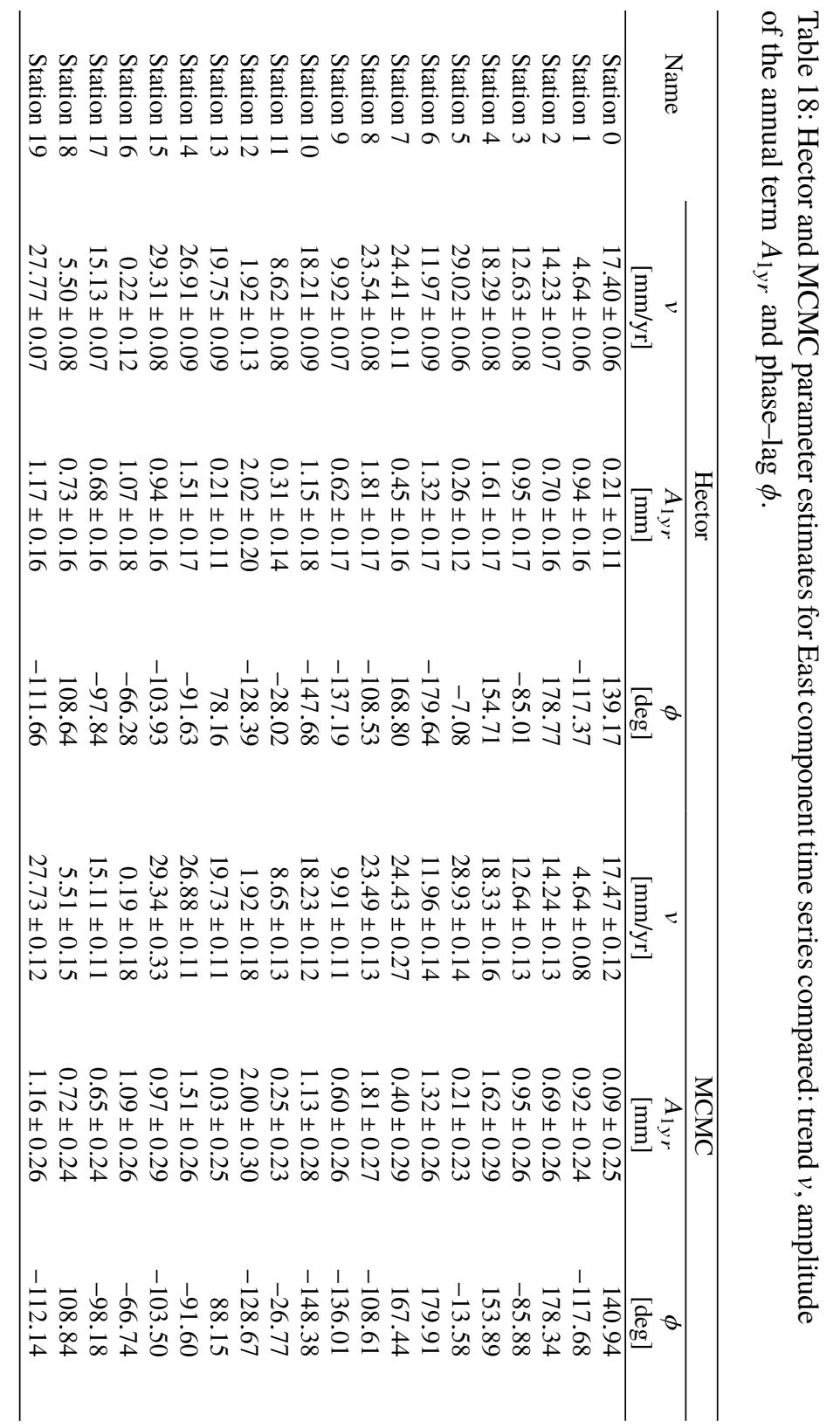




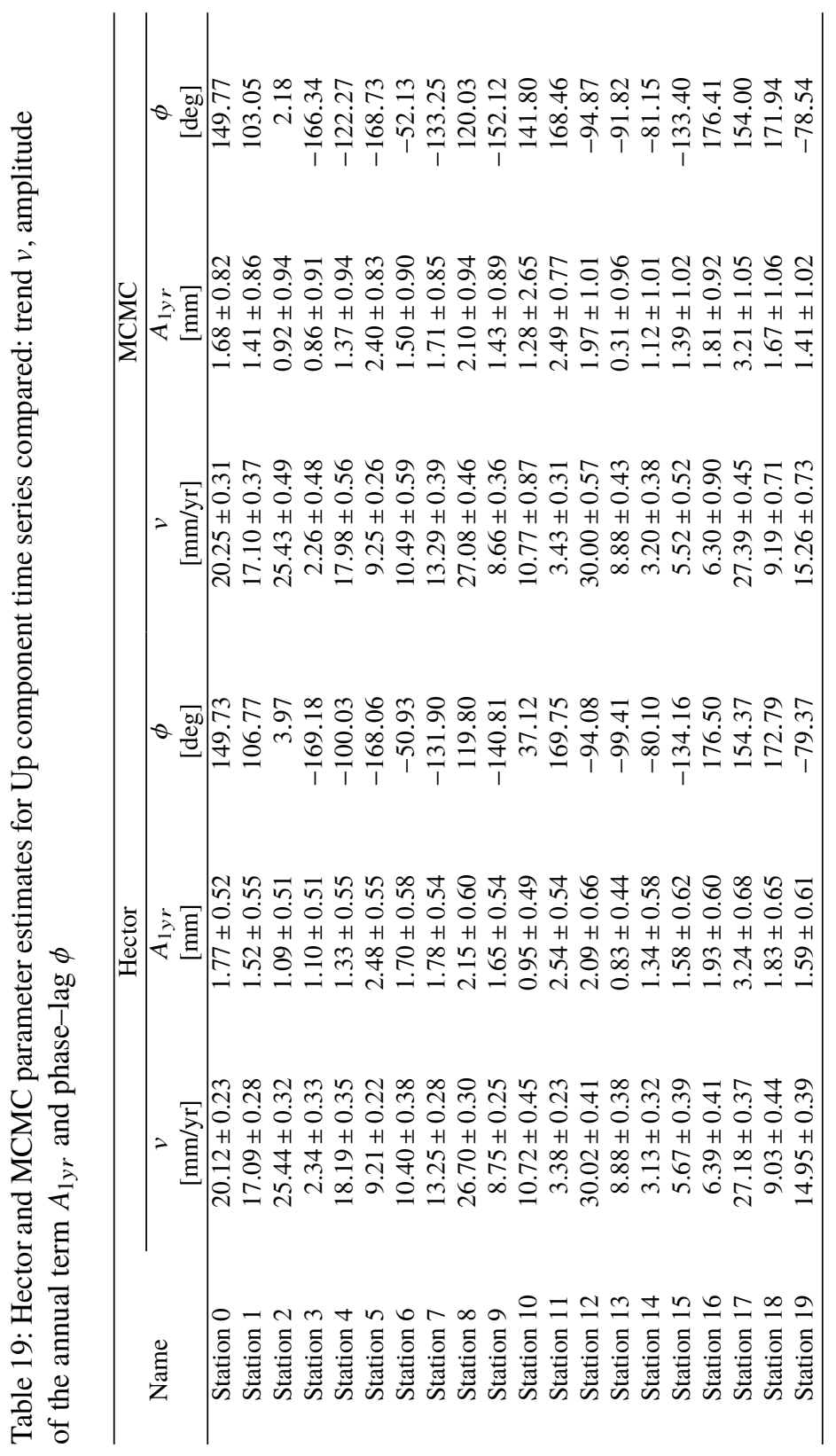



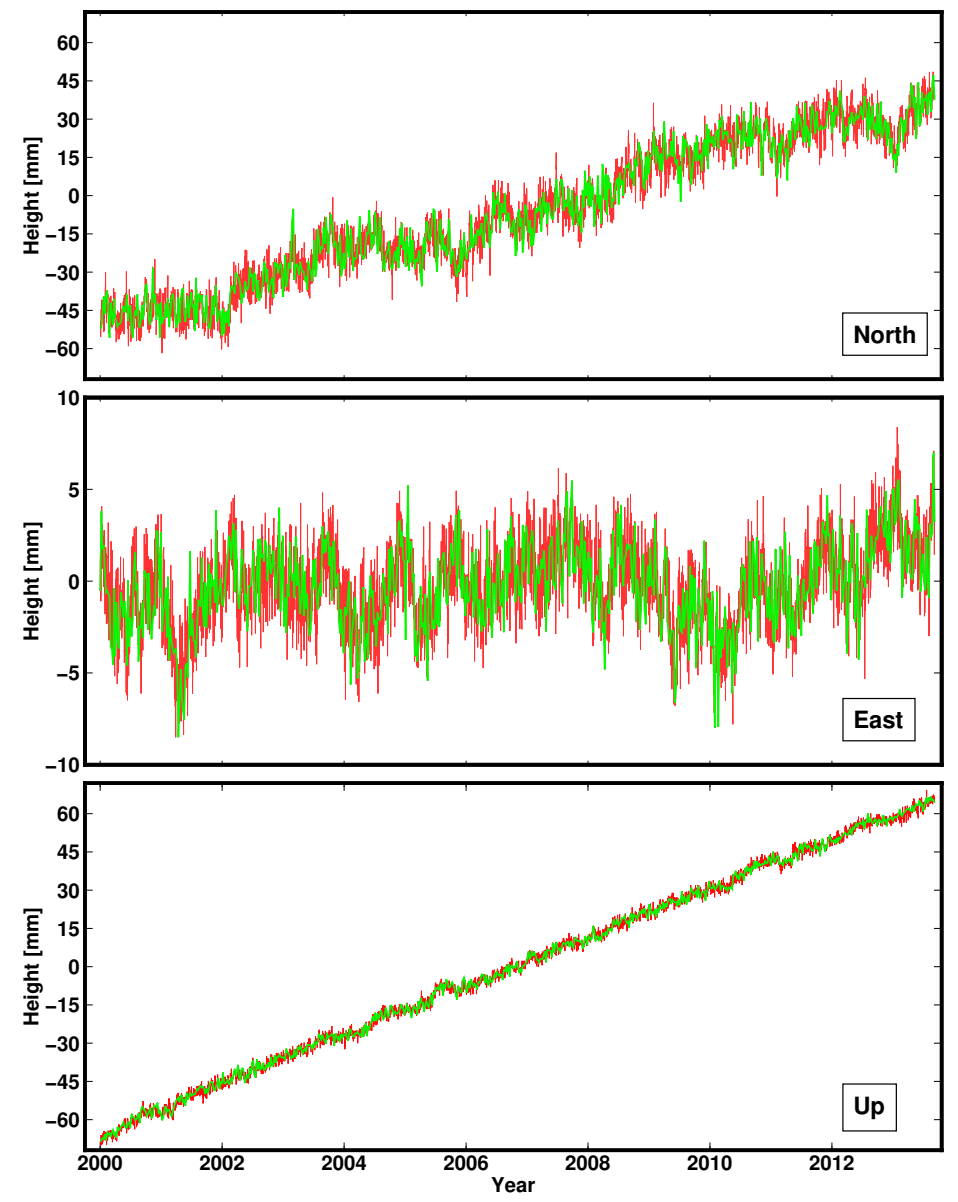

Fig. 36: A weekly sampled time series (green line) superimposed on the daily time series (red line) for one of the time series for North, East, and Up components. 

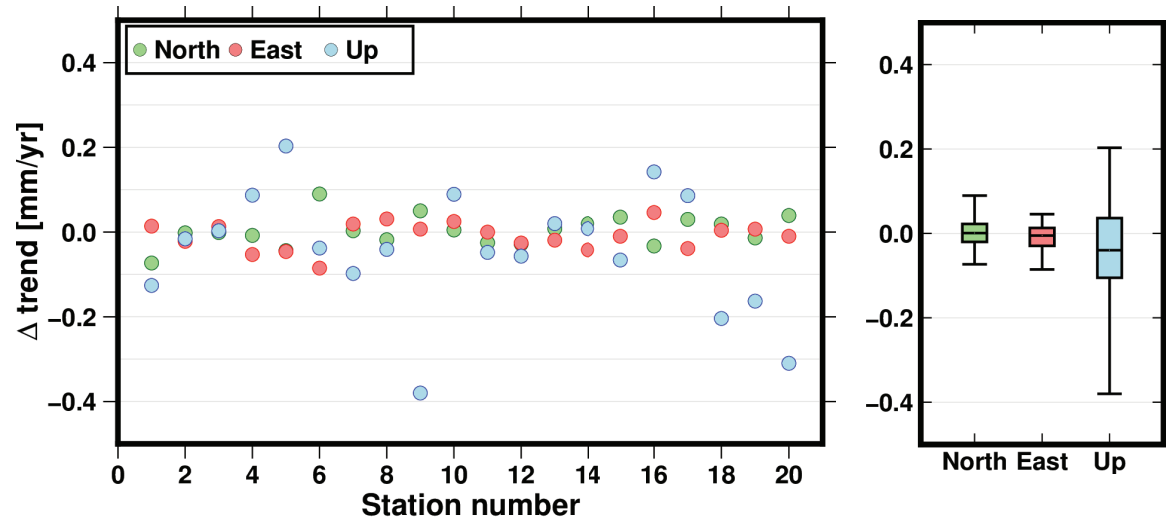

Fig. 37: Trend differences between Hector and MCMC for the 20 weekly BSG time series. North, East, and Up components are displayed in green, red, and blue, respectively. A box whisker plot showing minimum, $25^{\text {th }}$ percentile, median, $75^{\text {th }}$ percentile and maximum values is to the right. 


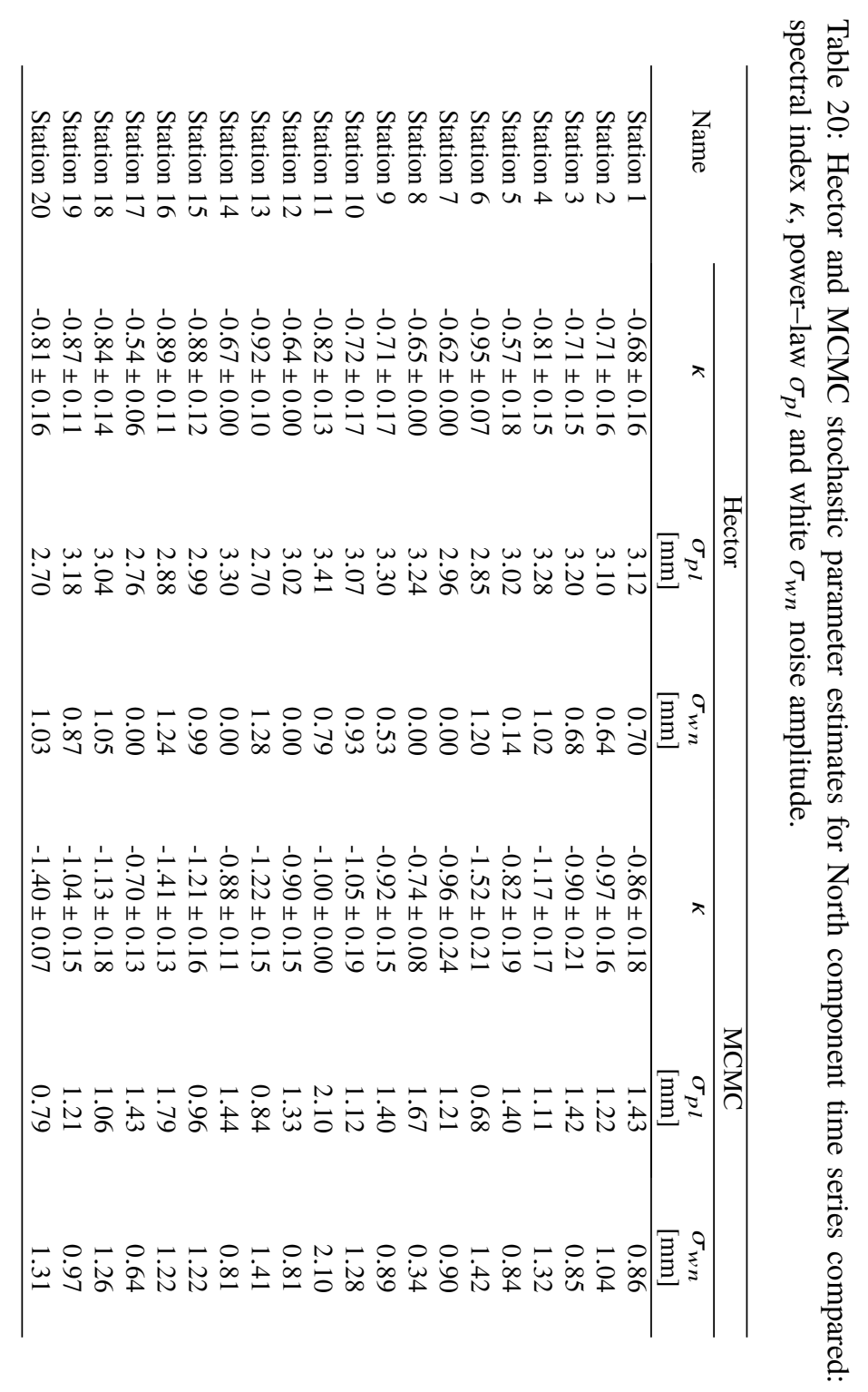




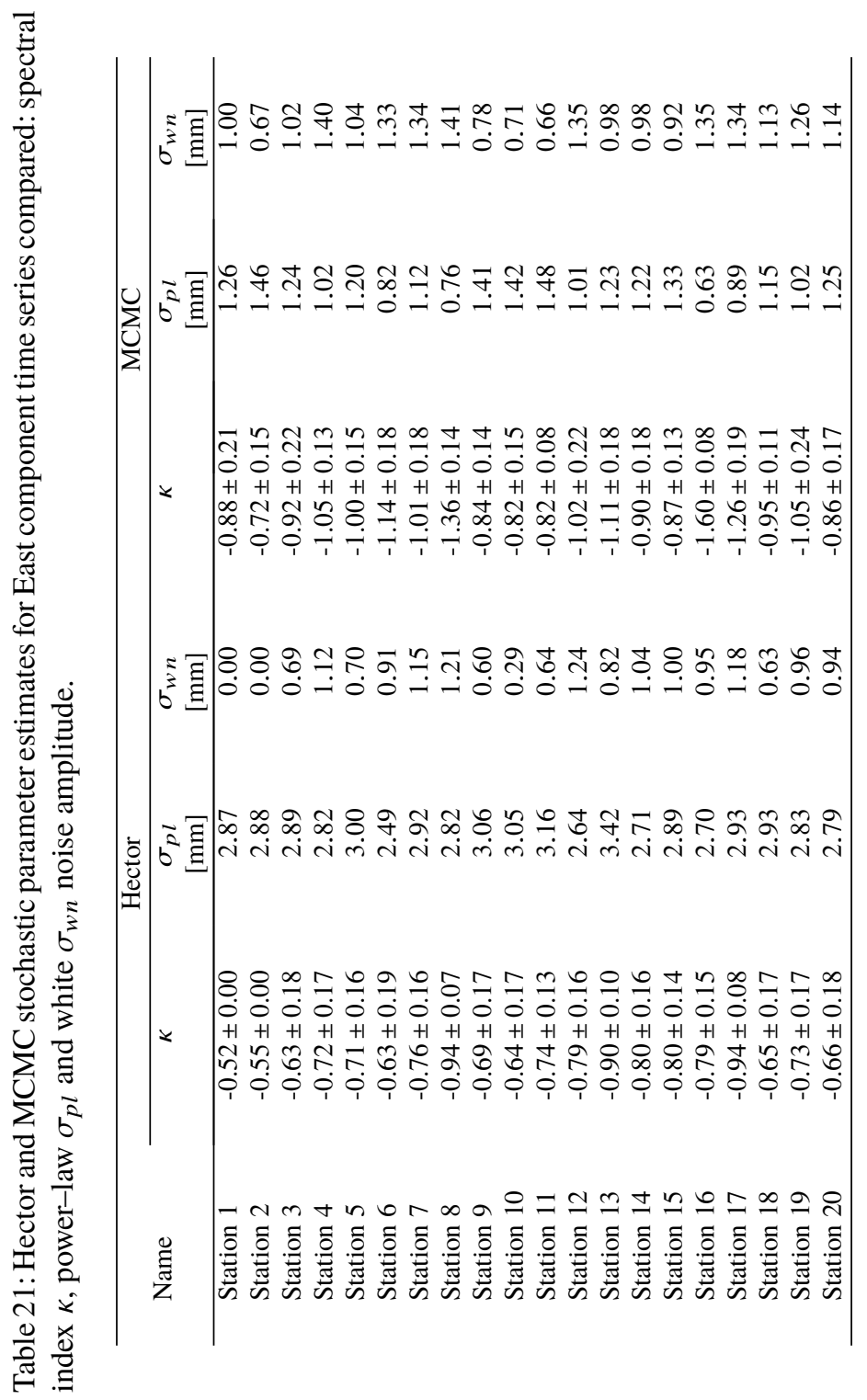




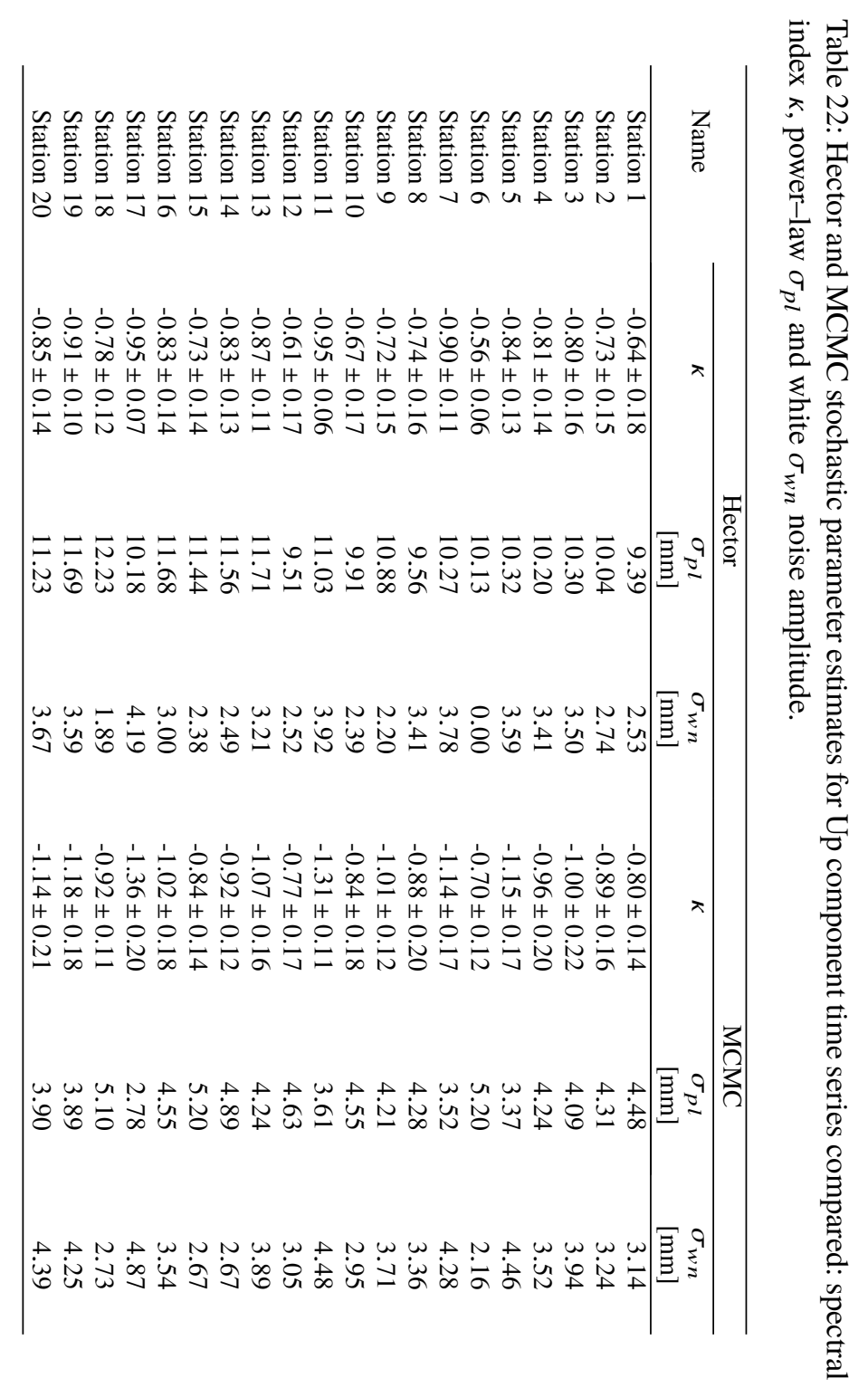




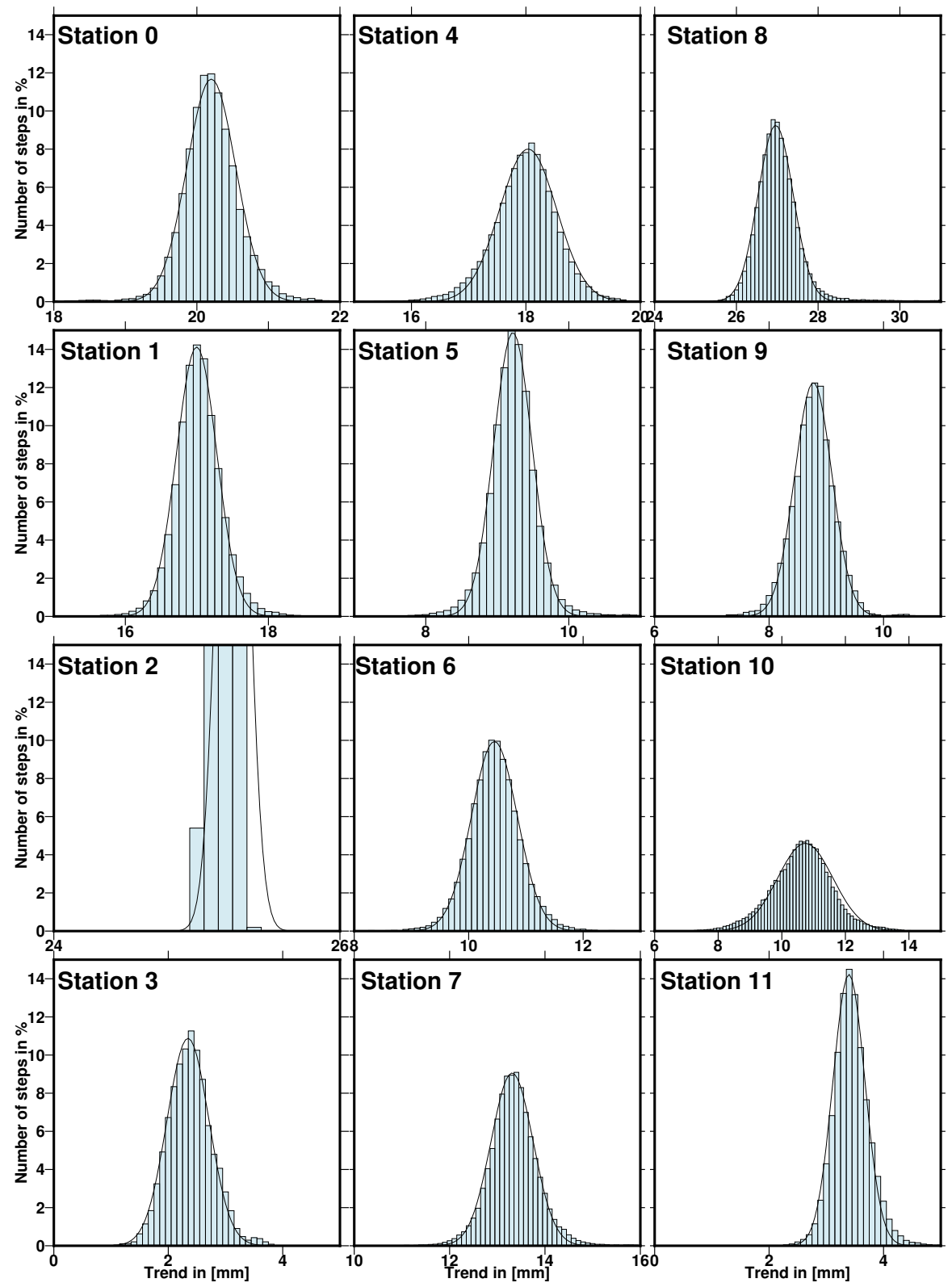

Fig. 38: Histograms of the trend estimates of the Up component, continued on next page 

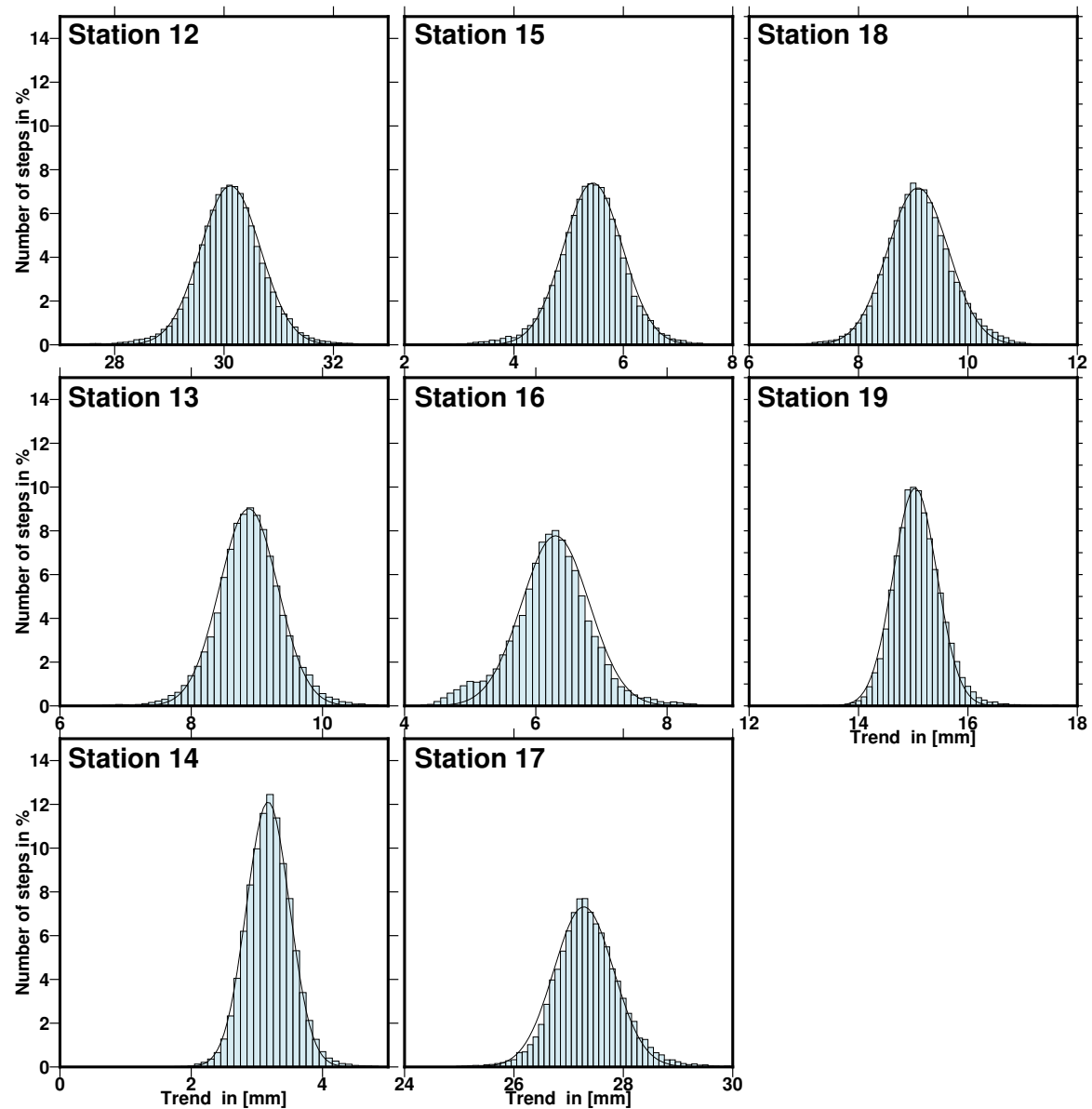

Fig. 39: Histograms of the trend estimates of the Up component, continued from Figure 38 

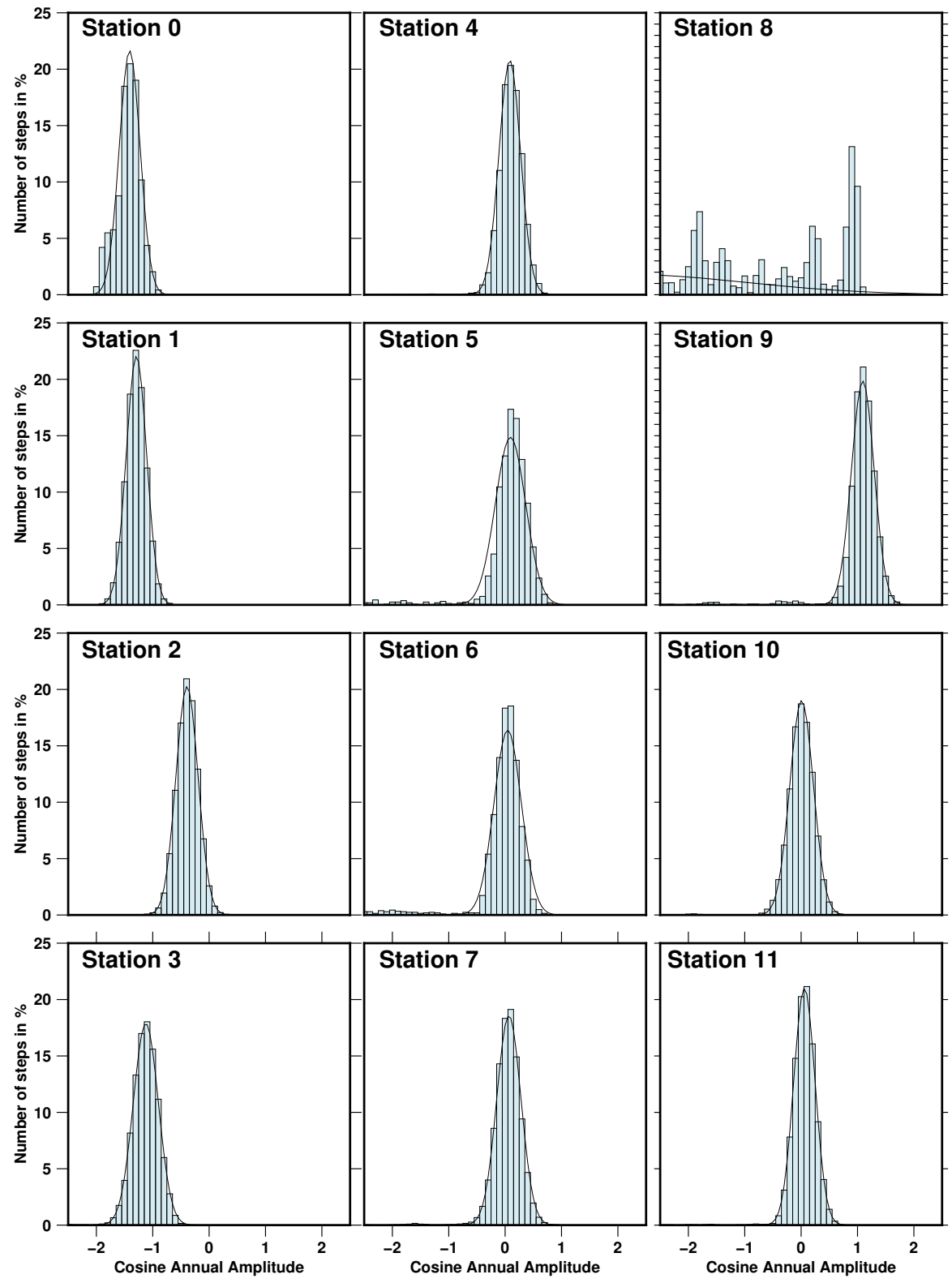

Fig. 40: Histograms for the annual term amplitude of the North component, continued on next page. 

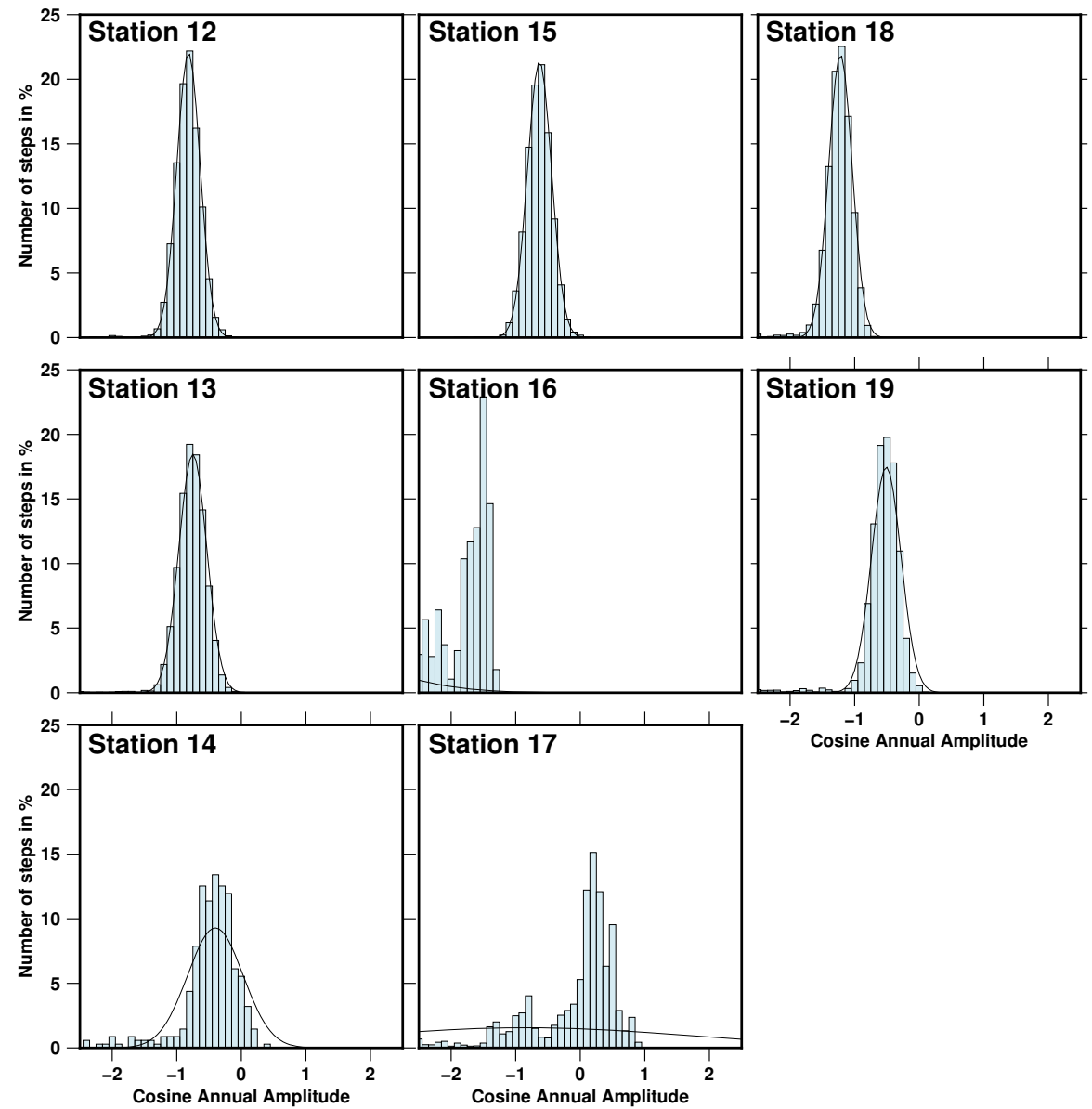

Fig. 41: Histograms for the annual term amplitude of the North component, continued from Figure 40. 

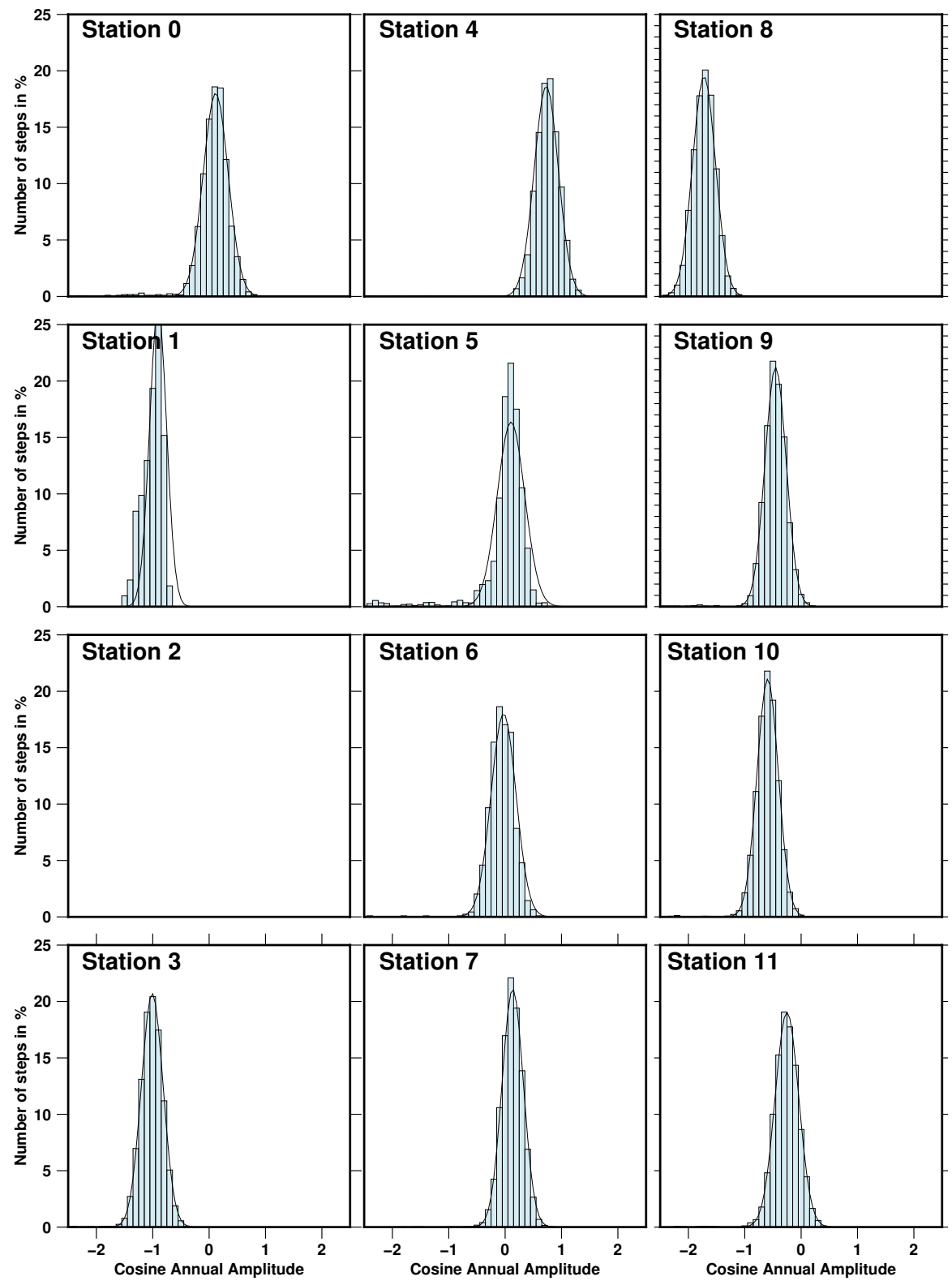

Fig. 42: Histograms for the annual term amplitude of the East component, continued on next page. 

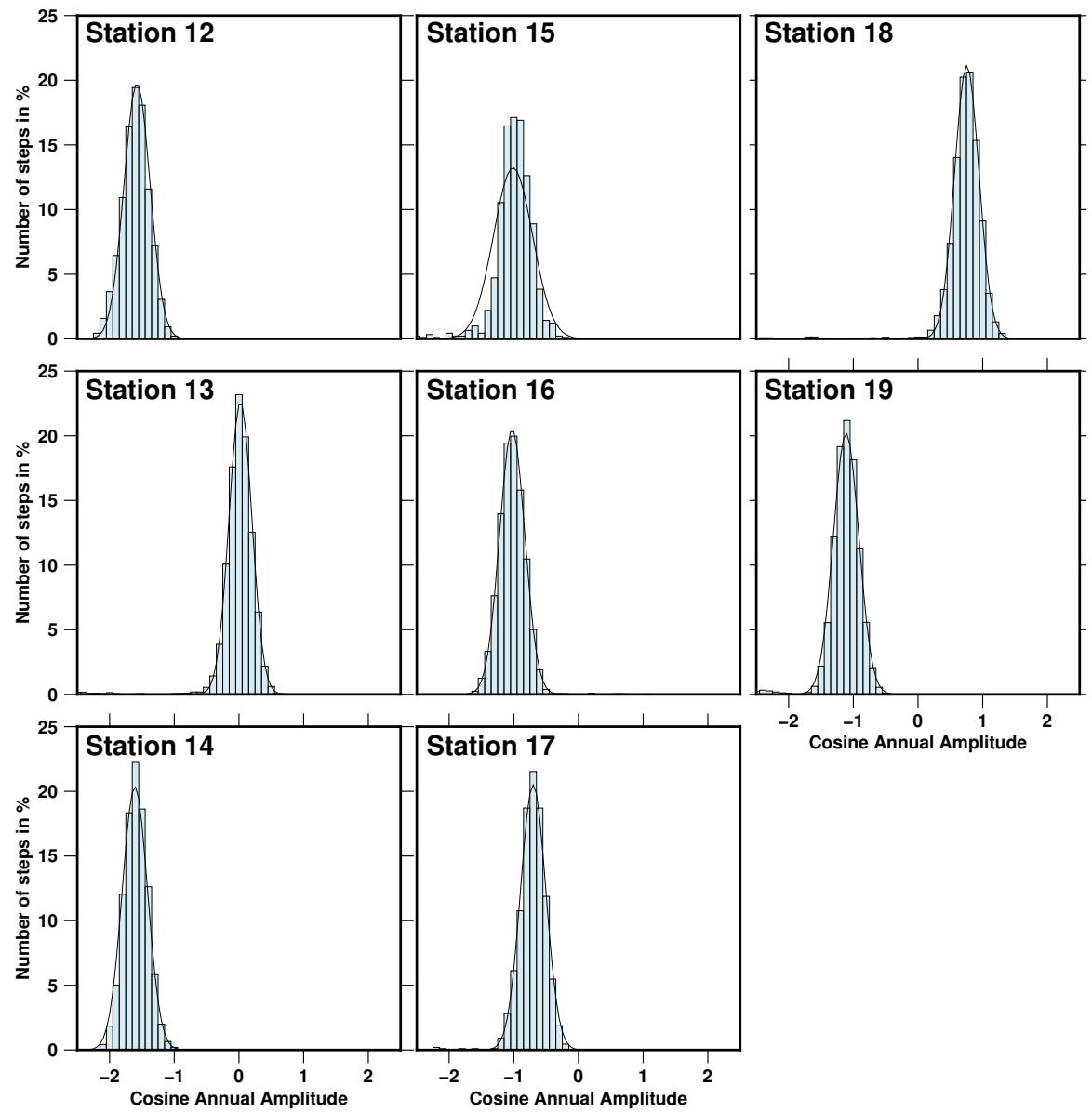

Fig. 43: Histogram for the annual term amplitude of the East component, continued from Figure 42. 

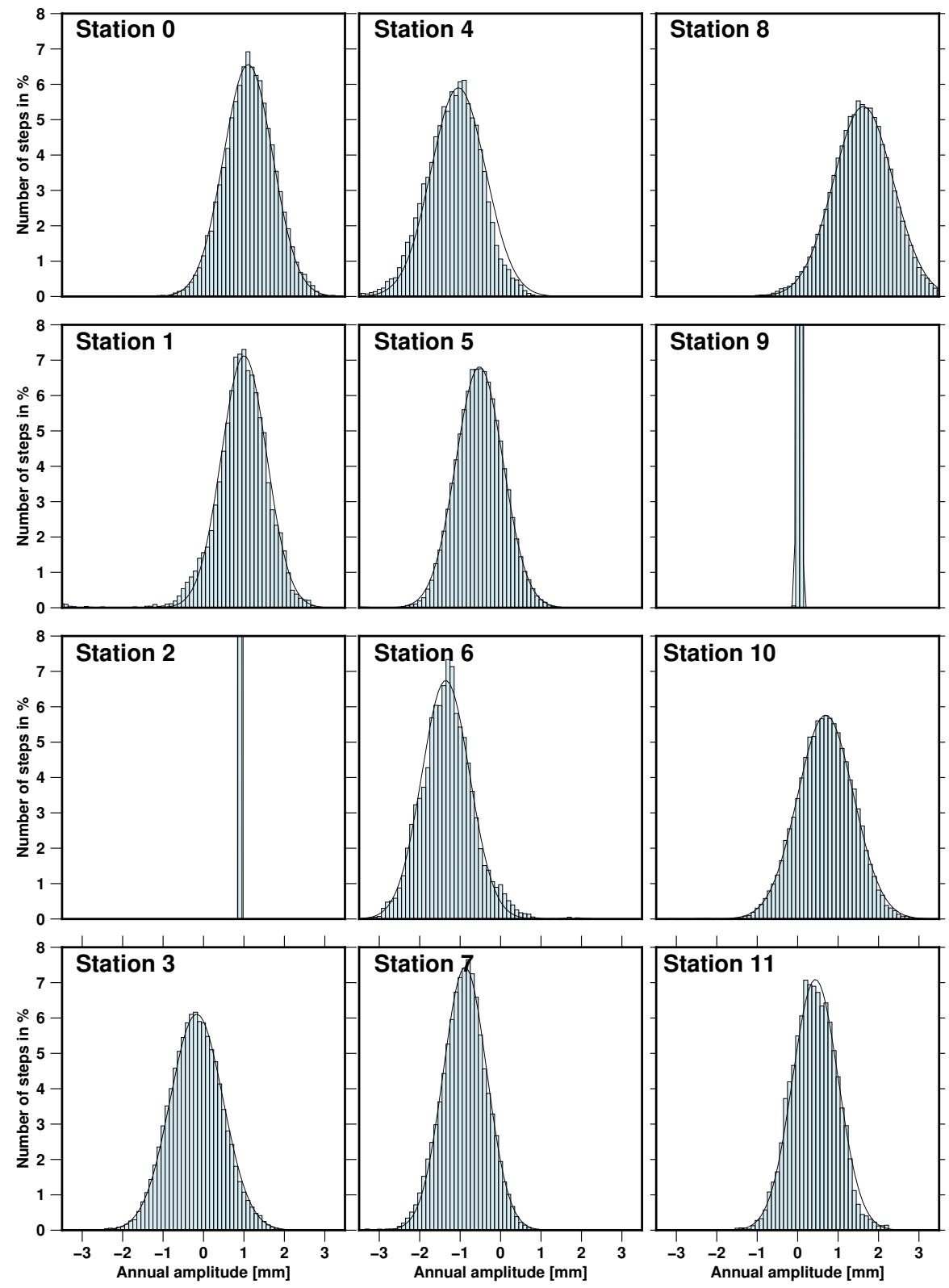

Fig. 44: Histograms for the annual term amplitude of the Up component, continued on next page. 

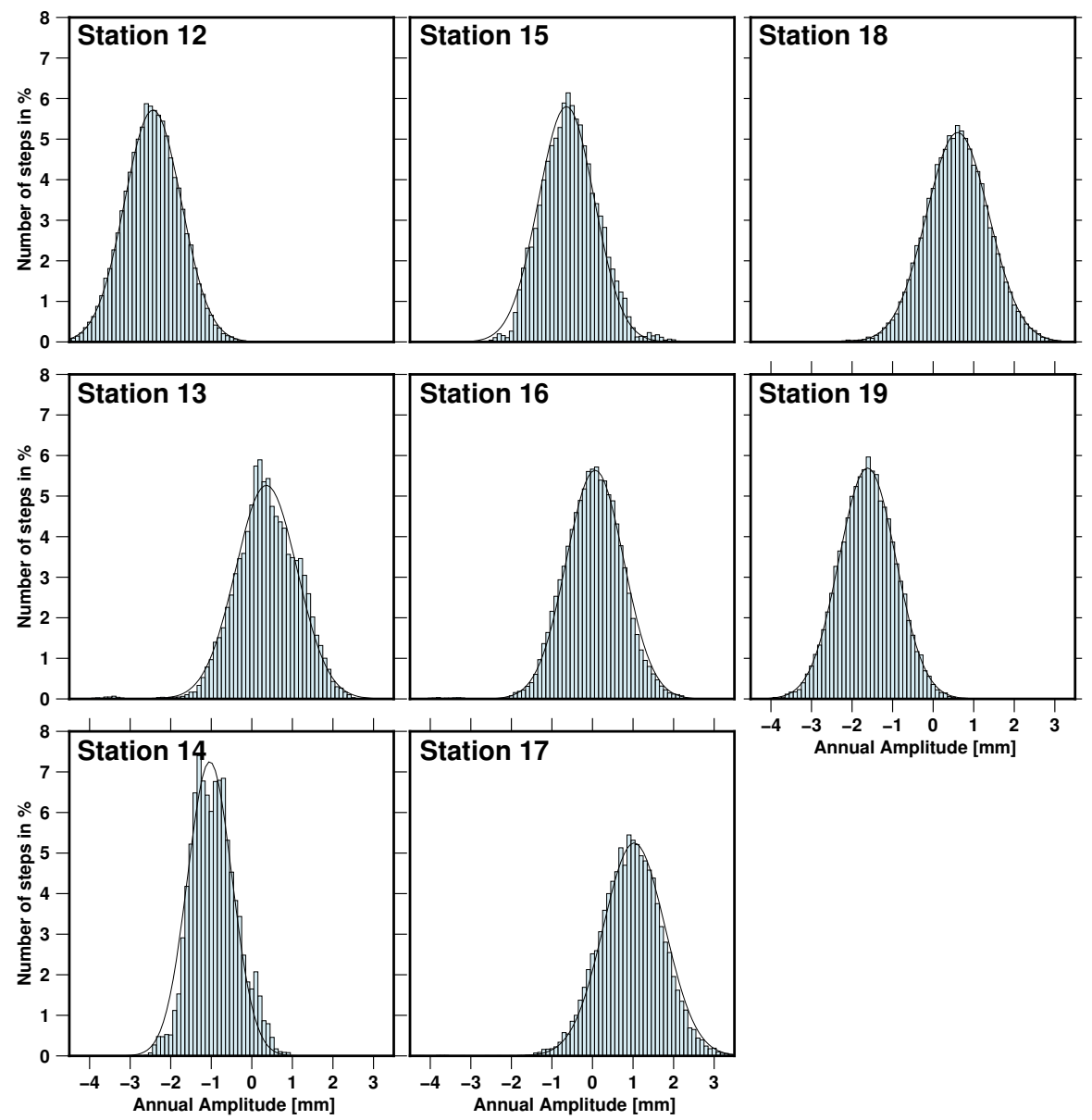

Fig. 45: Histogram for the annual term amplitude of the Up component, continued from Figure 44. 


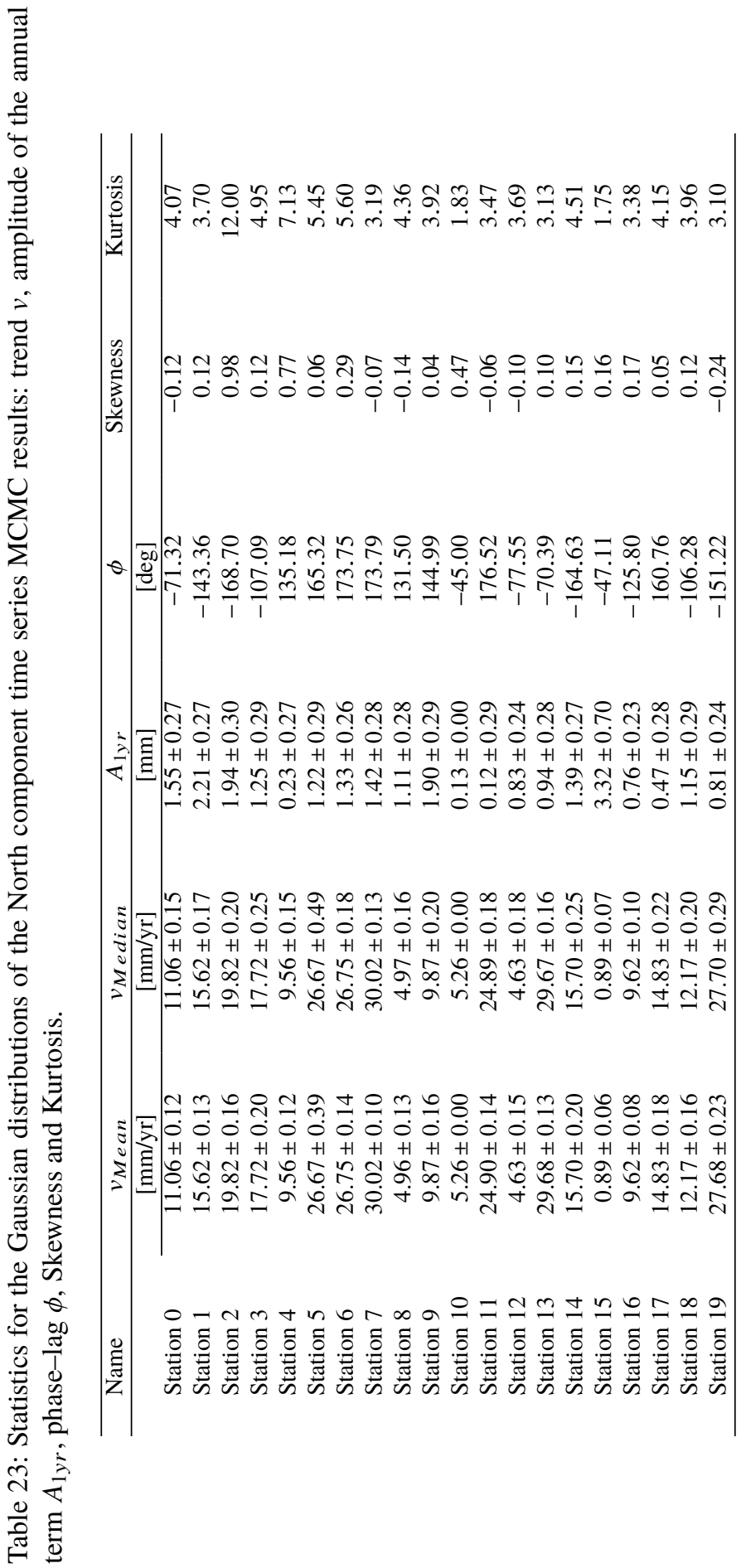




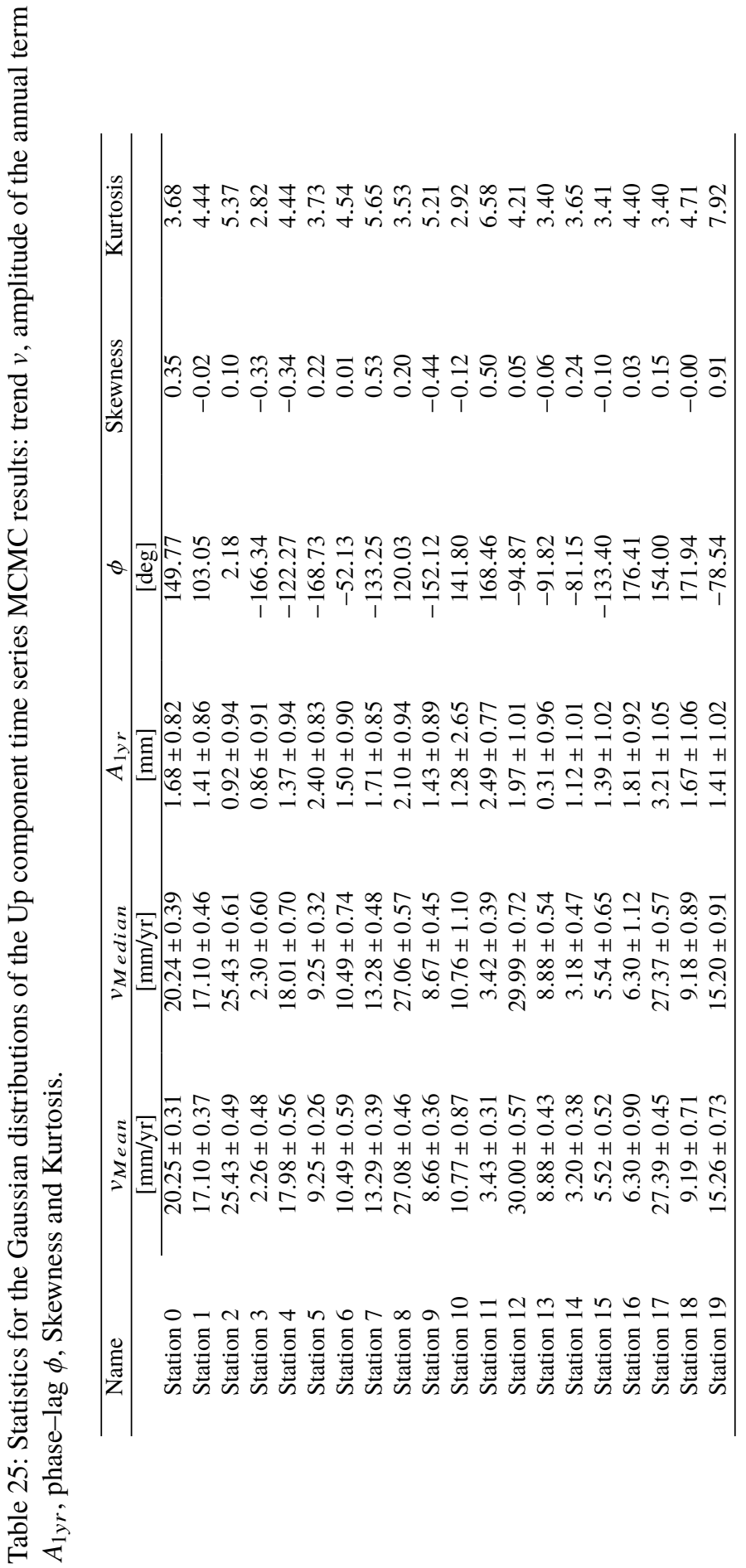




\section{References}

Agnew, D., February 1992. The time-domain behaviour of power-law noises. Geophys. Res. Lett. 19 (4), 333-336.

Altamimi, Z., Métivier, L., Collilieux, X., 2012. ITRF2008 plate motion model. J. Geophys. Res. 117, B07402.

Argus, D. F., Heflin, M. B., Peltzer, G., Crampe, F., Webb, F. H., 2005. Interseismic strain accumulation and anthropogenic motion in metropolitan los angeles. $\mathrm{J}$. Geophys. Res. 110, B04401, doi: 10.1029/2003JB002934.

Baart, F., van Gelder, P., de Ronde, J., van Koningsveld, M., Wouters, B., 2012. The effect of the 18.6-year lunar nodal cycle on regional sea-level rise estimates. J. Coastal Res. 28 (2), 511-516.

Beutler, G., Rothacher, M., Schaer, S., Springer, T., Kouba, J., Neilan, R., 1999. The International GPS Service (IGS): an interdisciplinary Service in support of earth sciences. Adv. Space Res. 23 (4), 631-635.

Blewitt, G., Lavallée, D., July 2002. Effect of annual signals on geodetic velocity. J. Geophys. Res. 107 (B7), ETG 9-1-ETG 9-11.

Bloßfeld, M., Rudenko, S., Kehm, A., Panafidina, N., Müller, H., Angermann, D., Hugentobler, U., Seitz, M., 2018. Consistent estimation of geodetic parameters from slr satellite constellation measurements. J. Geodesy 92 (9), 1003-1021.

Bos, M., Bastos, L., Fernandes, R., 2010. The influence of seasonal signals on the estimation of the tectonic motion in short continuous GPS time-series. J. Geodyn. (49), 205-209.

Bos, M., Fernandes, R., Williams, S., Bastos, L., December 2012. Fast error analysis of continuous GNSS observations with missing data. J. Geodesy 87, 351-360.

Bradley, S. L., Milne, G. A., Teferle, F. N., Bingley, R. M., Orliac, E. J., 2009. Glacial isostatic adjustment of the british isles: New constraints from gps measurements of crustal motion. Geophys. J. Int. 178 (1), 14-22.

Burgette, R., Watson, C., Church, J., White, N., Tregoning, P., Coleman, R., 2013. Characterizing and minimizing the effects of noise in tide gauge time series: relative and geocentric sea level rise around Australia. Geophys. J. Int. doi:10.1093/gji131.

Caporali, A., 2003. Average strain rate in the Italian crust inferred from a permanent GPS network - I. Statistical analysis of the time-series of permanent GPS stations. Geophys. J. Int. (155), 241-253.

Cazenave, A., Valette, J., Boucher, C., 1992. Positioning results with DORIS on SPOT2 after first year of mission. J. Geophys. Res. 97 (B5), 7109-7119.

Chambers, D., Merrifield, M., Nerem, R., 2012. Is there a 60-year oscillation in global mean sea level? Geophys. Res. Lett. 39 (doi:10.1029/2012GL052885).

Church, J., White, N., 2006. A 20th century acceleration in global sea-level rise. Geophys. Res. Lett. 33, L01602.

Douglas, B., Kearney, M., Leatherman, S., 2001. Sea Level Rise. Vol. 75. Academic Press.

Dow, J., Neilan, R., Rizos, C., 2009. The International GNSS Service in a changing landscape of Global Navigation Satellite Systems. J. Geodesy 83 (3), 191-198. 
Dunkley, J., Bucher, M., Ferreira, P. G., Moodley, K., Skordis, C., 2005. Fast and reliable MCMC for cosmological parameter estimation. Mon. Not. R. Astron. Soc. 356, 925-936.

Fernandes, R. M. S., Ambrosius, B., Noomen, R., Bastos, L., Combrinck, L., Miranda, J. M., Spakman, W., 2004. Angular velocities of nubia and somalia from continuous gps data: implications on present-day relative kinematics. Earth Planet. Sc. Lett. 222, 197-208.

Gazeaux, J., Williams, S., Matt, K., Bos, M., Dach, R., Deo, M., Moore, A., Ostini, L., Petrie, E., Roggero, M., Teferle, F., Olivares, G., Webb, F., 2013. Detecting offsets in GPS time series: First results from the detection of offsets in GPS experiment. J. Geophys. Res. 118 (doi:10.1002/jgrb.50152.).

Gilks, W. R., Richardson, S., Spiegelhalter, D., 1996. Markov Chain Monte Carlo in Practice. Chapman \& Hall.

Goodkind, J., 1999. The superconducting gravimeter. Rev. Sci. Instrum. 70 (11), 4131-4152.

Hackl, M., Malservisi, R., Hugentobler, U., Wonnacott, R., 2011. Estimation of velocity uncertainties from GPS time series: Examples from the analysis of the South African TrigNet network. J. Geophys. Res. 116, B11404.

Harrison, C., 2002. Power spectrum of sea level change over fifteen decades of frequency. Geochem. Geophys. Geosys. 3 (8), 1-17.

Holgate, S., Matthews, A., Woodworth, P., Rickards, L., Tamisiea, M., Bradshaw, E., Foden, P., Gordon, K., Jevrejeva, S., Pugh, J., 2013. New Data Systems and Products at the Permanent Service for Mean Sea Level. J. Coastal Res. 29 (3), 493 -504 .

Holgate, S., Woodworth, P., 2004. Evidence for enhanced coastal level rise during the 1990s. Geophys. Res. Lett. 31, L07305.

Hosking, J. R. M., April 1981. Fractional differencing. Biometrika 68 (1), 165-176.

Hughes, C., Williams, S., 2010. The color of sea level: Importance of spatial variations in spectral shape for assessing the significance of trends. J. Geophys. Res. 115 (doi:10.1029/2010JC006102).

Khan, S. A., Wahr, J., Leuliette, E., van Dam, T., Larson, K. M., Francis, O., 2008. Geodetic measurements of postglacial adjustments in greenland. J. Geophys. Res. 113.

URL http://dx.doi.org/10.1029/2007JB004956

Kirchner, J., 2005. Aliasing in $1 / f^{\alpha}$ noise spectra: Origins, consequences, and remedies. Phys. Rev. E 71 (066110).

Klos, A., Hunegnaw, A., Teferle, F. N., Abraha, K. E., Ahmed, F., Bogusz, J., 2018a. Statistical significance of trends in zenith wet delay from re?processed gps solutions. GPS Solut. 22 (51), doi: 10.1007/s10291-018-0717-y.

Klos, A., Olivares, G., Teferle, F. N., Hunegnaw, A., Bogusz, J., 2018b. On the combined effect of periodic signals and colored noise on velocity uncertainties. GPS Solut. 22 (1), 1.

Langbein, J., 2004. Noise in two-color electronic distance meter measurements revisited. J. Geophys. Res. 109 (B04406). 
Langbein, J., 2008. Noise in GPS displacement measurements from Southern California and Southern Nevada. J. Geophys. Res.: Solid Earth 113, doi:10.1029/2007JB005247.

Langbein, J., Johnson, H., January 10 1997. Correlated errors in geodetic time series: Implications for time-dependent deformation. J. Geophys. Res. 102 (B1), 591-603.

Langbein, J., Quilty, E., Breckenridge, K., 1993. Sensivity of crustal deformation instruments to changes in secular rate. Geophys. Res. Lett. 20, 85-88.

Larson, K. M., Agnew, D. C., 1991. Application of the global positioning system to crustal deformation measurement 1. precision and accuracy. J. Geophys. Res. 96 (B10), 16547-16565.

Lefebvre, M., Cazenave, A., Escudier, P., Biancale, R., Crétaux, J., Soudarin, L., Valette, J., 1996. Space tracking system improves accuracy of geodetic measurements. Eos Trans. Am. Geophys. Union 77 (4), 25-29.

Mandelbrot, B. B., 1982. The Fractal Geometry of Nature. W.H.Freeman \& Co Ltd.

Mao, A., Harrison, C., Dixon, T., February 1999. Noise in gps coordinate time series. J. Geophys. Res. 104 (B2), 2797-2816.

Mazzotti, S., Jones, C., R.E., T., 2008. Relative and absolute sea level rise in western Canada and northwestern United States from a combined tide gauge-GPS analysis. J. Geophys. Res. 113 (C11019), doi:10.1029/2008JC004835.

Metropolis, N., Rosenbluth, A., Rosenbluth, M., Teller, A., Teller, E., June 1953. Equation of state calculations by fast computing machines. J. Chem. Phys. 21 (6), 1087-1093.

Milne, G. A., Davies, J. L., Mitrovica, J. X., Scherneck, H. G., Johansson, J. M., Vermeer, M., Koivula, H., 2001. Space-geodetic constraints on glacial isostatic adjustment in fennoscandia. Science 291 (23 March 2001), 2381-2385.

Nahmani, S., Bock, O., Bouin, M.-N., Santamaría-Gómez, A., Boy, J.-P., Collilieux, X., Métivier, L., Panet, I., Genthon, P., de Linage, C., Wöppelmann, G., 2012. Hydrological deformation induced by the West African Monsoon: Comparison of GPS, GRACE and loading models. J. Geophys. Res.: Solid Earth 117 (B5), B05409.

Nielsen, K., Khan, S. A., Spada, G., Wahr, J., Bevis, M., Liu, L., van Dam, T., 2013. Vertical and horizontal surface displacements near Jakobshavn Isbrae driven by melt-induced and dynamic ice loss. J. Geophys. Res.: Solid Earth 118 (4), 18371844.

Nothnagel, A., Artz, T., Behrend, D., Malkin, Z., 2017. International vlbi service for geodesy and astrometry. J. Geodesy 91 (7), 711-721.

Olivares, G., Teferle, F., May 2013. A bayesian monte carlo markov chain method for parameter estimation of fractional differenced gaussian processes. IEEE Trans. Signal Process. 61 (9), 2405-2412.

Pearlman, M., Degnan, J., Bosworth, J., 2002. The International Laser Ranging Service. Adv. Space Res. 30 (2), 135-142.

Prawirodirdjo, L., Bock, Y., McCaffrey, R., Genrich, J., Calais, E., Stevens, C., Puntodewo, S. S. O., Subarya, C., Rais, J., Zwick, P., Fauzi, 1997. Geodetic 
observations of interseismic strain segmentation at the sumatra subduction zone. Geophys. Res. Lett. 24 (21), 2601-2604.

Roberts, G. O., Rosenthal, J. S., November 2001. Optimal scaling for various metropolis-hastings algorithms. Stat. Sci. 16 (4), 351-367.

Santamaría-Gómez, A., Gravelle, M., Collilieux, X., Guichard, M., Martín Míguez, B., Tiphaneau, P., Wöppelmann, G., 2012. Mitigating the effects of vertical land motion in tide gauge records using a state-of-the-art GPS velocity field. Global Planet. Change 98-99, 6-17.

Schlüter, W., Himwich, E., Nothnagel, A., Vandenberg, N., Whitney, A., 2002. IVS and its important role in the maintenance of the global reference systems. Adv. Space Res. 30 (2), 145-150.

Schwarz, G., 1978. Estimating the dimension of a model. Ann. Stat. 6 (2), 461-464.

Teferle, F., Bingley, R., Dodson, A., Penna, N. T., Baker, T. F., 2002. Using GPS to separate crustal movements and sea level changes at tide gauges in the UK. International Association of Geodesy Symposia, Vol 124. Springer-Verlag, Heidelberg Berlin, pp. 264-269.

Teferle, F. N., Bingley, R. M., Orliac, E. J., Williams, S. D. P., Woodworth, P., McLaughlin, D., Baker, T. F., Shennan, I., Milne, G. A., Bradley, S. L., 2009. Crustal motions in great britain: Evidence from continuous gps, absolute gravity and holocene sea-level data. Geophys. J. Int. 178 (1), 23-46.

Teferle, F. N., Williams, S., Kierulf, H. P., Bingley, R., Plag, H.-P., 2008. A continuous gps coordinate time series analysis strategy for high-accuracy vertical land movements. Phys. Chem. Earth 33 (3-4), 205-216, doi:10.1016/j.pce.2006.11.002.

Teke, K., Böhm, J., Nilsson, T., Schuh, H., Steigenberger, P., Dach, R., Heinkelmann, R., Willis, P., Haas, R., Garcia-Espada, S., Hobiger, T., Ichikawa, R., Shimizu, S., 2011. Multi-technique comparison of troposphere zenith delays and gradients during cont08. J. Geodesy 85, 395-413.

Van Camp, M., De Viron, O., Watlet, A., Meurers, B., Francis, O., Caudron, C., 2017. Geophysics from terrestrial time-variable gravity measurements. Reviews of Geophysics 55, 938-992.

Van Camp, M., Meurers, B., de Viron, O., Forbirger, T., 2016. Optimized strategy for the calibration of superconducting gravimeters at the one per mille level. J. Geodesy 90 (1), 91-99.

van Camp, M., Vanclooster, M., Crommen, O., Petermans, T., Verbeeck, K., Meurers, B., van Dam, T., Dassargues, A., 2006. Hydrological investigations at the membach station, belgium, and application to correct long periodic gravity variations. J. Geophys. Res. 111, B10403, doi: 10.1029/2006JB004405.

Van Camp, M., Williams, S., Francis, O., 2005. Uncertainty of absolute gravity measurements. J. Geophys. Res. 110 (B05406).

Virtanen, H., 2004. Loading effects in metsahovi from the atmosphere and the baltic sea. J. Geodyn. 38 (3-5), 407-422.

URL http://www.sciencedirect.com/science/article/B6V9X-4DN1H6C1/2/4e25c66d6e0ea5da2bf5cc8e1d7f0373

White, N., Church, J., Gregory, J., 2005. Coastal and global averaged sea level rise for 1950 to 2000. Geophys. Res. Lett. 32, L01601. 
Williams, S., 2003a. The effect of coloured noise on the uncertainties of rates estimated from geodetic time series. J. Geodesy (76), 483-494.

Williams, S., 2003b. Offsets in global positioning system time series. J. Geophys. Res 108 (B6), 2310.

Williams, S., 2008. CATS: GPS coordinate time series analysis software. GPS Solut. (12), 147-153.

Williams, S., Bock, Y., Fang, P., Jamason, P., Nikolaidis, R., Miller, M., Johnson, D., 2004. Error analysis of continuous GPS position time series. J. Geophys. Res. 109 (B03).

Williams, S., Willis, P., 2006. Error analysis of weekly station coordinates in the DORIS network. J. Geodesy 80 (8-11), 525-539.

Willis, P., Fagard, H., Ferrage, P., Lemoine, F., Noll, C., Noomen, R., Otten, M., Ries, J., Rothacher, M., Soudarin, L., Tavernier, G., Valette, J., 2010. The International DORIS Service (IDS): Toward maturity, in DORIS: Scientific Applications in Geodesy and Geodynamics. Adv. Space Res. 45 (12), 1408-1420.

Woodworth, P., Teferle, F. N., Bingley, R. M., Shennan, I., Williams, S. D. P., 2009. Trends in uk mean sea level revisited. Geophys. J. Int. 176 (1), 19-30.

Woodworth, P., Tsimplis, M., Flather, R., Shennan, I., 1999. A review of the trends observed in British Isles mean sea level data measured by tide gauges. Geophys. J. Int. 136, 651-670.

Wöppelmann, G., Letetrel, C., Santamaría-Gómez, A., Bouin, M.-N., Collilieux, X., Altamimi, Z., Williams, S., Martín Míguez, B., 2009. Rates of sea-level change over the past century in a geocentric reference frame. Geophys. Res. Lett. 36 (L12607).

Wöppelmann, G., Martín Míguez, B., Bouin, M.-N., Altamimi, Z., 2007. Geocentric sea-level trend estimates from gps analyses at relevant tide gauges world-wide. Global Planet. Change 57, 396-406.

Wyatt, F., 1982. Displacement of surface monuments: horizontal motion. J. Geophys. Res. (87), 979-989.

Wyatt, F., 1989. Displacement of surface monuments: vertical motion. J. Geophys. Res. (94), 1655-1664.

Zhang, J., Bock, Y., Fang, P., Williams, S., Genrich, J., Wdowinski, S., Behr, J., August 1997. Southern california permanent gps geodetic array: Error analysis of daily position estimates and site velocities. J. Geophys. Res. 102 (B8), 18,03518,055 .

Zumberge, J., Heflin, M., Jefferson, D., Watkins, M., Webb, F., March 1997. Precise point positioning for the efficient and robust analysis of GPS data from large networks. J. Geophys. Res. 102 (B3), 5005-5017. 